\title{
Real-Time Path Planning for Needle Insertion With Multiple Targets
}

\author{
by \\ Afsoon Nejati Aghdam, B. Eng., M. Eng. (Research) \\ A Thesis submitted to \\ the Faculty of Graduate and Postdoctoral Affairs \\ in partial fulfillment of \\ the requirements for the degree of \\ Doctor of Philosophy \\ in \\ Systems and Computer Engineering \\ Ottawa-Carleton Institute for Electrical and Computer Engineering (OCIECE) \\ Department of Systems and Computer Engineering \\ Carleton University \\ June 2020 \\ Copyright (C) \\ 2020 - Afsoon Nejati Aghdam
}


The undersigned recommend to

the Faculty of Graduate and Postdoctoral Affairs

acceptance of the Thesis

\title{
Real-Time Path Planning for Needle Insertion With Multiple Targets
}

\author{
Submitted by Afsoon Nejati Aghdam \\ in partial fulfilment of the requirements for the degree of \\ Doctor of Philosophy
}

Professor Peter X. Liu, Supervisor

Ottawa-Carleton Institute for Electrical and Computer Engineering (OCIECE)

Department of Systems and Computer Engineering

Carleton University 


\section{Abstract}

Needle insertion into soft tissue has gained considerable attention in recent years in medical applications due to its ever-increasing potential in minimally invasive procedures. Steerable bevel-tip needles offer higher maneuverability independent of the insertion depth and, consequently, are preferable in many needle steering applications compared to symmetric-tip needles. However, due to the nonholonomic kinematics of the bevel-tip needle inside soft tissue, its path planning poses a considerable challenge. Though the topic of single-target path planning is rather well studied and researched, the multiple-target path-planning problem remains under-researched. In this work, we study the path-planning problem for multiple targets based on Rapidly-Exploring-Random-Tree (RRT) algorithms. These algorithms are proper candidates for intra-operative planning of needle motion due to their fast computation and simple implementations. They also work well in highdimensional configuration spaces and under nonholonomic kinematic constraints, both of which are the characteristics of steerable bevel-tip needle motion inside soft tissue. We present two novel RRT-based path-planning approaches to steerable bevel-tip needles to reach multiple targets inside soft tissue: a $2 \mathrm{D}$ path planner for preoperative applications and a 3D real-time path planner for intraoperative applications. In both planners, without the needle having to completely retract and reinsert toward each separate target, the amount of tissue damage compared to the conventional sequential insertion of the needle toward each target decreases significantly. Particularly, our 3D planner works well in real-world applications where tissue and anatomical structures may vary due to tissue deformation during insertion, patient's motion, or physiological changes. In addition, our 3D planner accounts for the needle's natural curvature variation during insertion due to tissue inhomogeneity.

Moreover, both of the proposed planners have real clinical applications, where 
the limited size of the workspace as well as the needle's limited natural curvature impose significant limitations on the needle's path-planning problem inside soft tissue. Unlike the optimization-based methods with exponential time complexity, our planners work well with as many targets as required. Simulations demonstrate the efficiency of the proposed planners in terms of minimum targeting error and decreased needle insertion length vis-a-vis the sequential insertion of the needle for each target. 
To my parents, for their eternal love. 


\section{Acknowledgments}

First, I thank my advisor Professor Peter Xiaoping Liu, who offered encouragement and advice all the way long. I will always look up to him as my academic role model. As always, I would also like to thank my family for their unfailing support during the long days when I was shackled to my computer keyboard. This work would not be as it is now without the generous help of my family. Also, I would like to thank my friends in the lab. I am indebted to you all - thank you. 


\section{Table of Contents}

Abstract $\quad$ iii

Acknowledgments $\quad$ vi

Table of Contents vii

List of Tables $\quad$ x

List of Figures $\quad$ xi

List of Acronyms xviii

List of Symbols $\quad$ xx

1 Introduction 1

1.1 Motivation . . . . . . . . . . . . . . . . . 1

1.2 Multiple-Target Path Planning . . . . . . . . . . . . . . . 4

1.3 Robotic Flexible Bevel-tip Needle Steering . . . . . . . . . . . . 5

1.3.1 Nonholonomic Kinematics of Bevel-Tip Needle . . . . . . . . . 6

1.3.2 Randomized Path-Planning Algorithms . . . . . . . . . . . . 7

1.3.3 Preoperative vs. Intraoperative Path Planning . . . . . . . . 8

1.4 Contributions ........................... 10

1.5 Thesis Organization . . . . . . . . . . . . . . . . . . 11 
2 Related Work $\quad 12$

2.1 Introduction . . . . . . . . . . . . . . . . . . . . . 12

2.2 Modeling Needle-Tissue Interaction . . . . . . . . . . . . . . . . . . 13

2.3 Needle Steering, Control, and Path Planning . . . . . . . . . . . . . . 21

2.3.1 Single Target . . . . . . . . . . . . . . . . . . . 24

2.3.2 Multi-Target Planning . . . . . . . . . . . . . . . 30

3 Needle Motion Kinematics through Soft Tissue 32

3.1 Introduction . . . . . . . . . . . . . . . . . . . 32

3.2 Considerations and assumptions . . . . . . . . . . . . . 33

3.3 Unicycle Model for Bevel-Tip Needle Motion . . . . . . . . . . . . . . 35

3.3.1 Planar Needle Motion and nonholonomic constraints . . . . . 36

3.3.2 Spatial Needle Motion . . . . . . . . . . . . . . . . . 38

3.3.3 Nonholonomic Constraints and Control Inputs . . . . . . . . . 39

3.4 Variable-Curvature Needle Motion . . . . . . . . . . . . . . . . . 43

3.4.1 Reachability Region . . . . . . . . . . . . . . . . . 43

3.4.2 Duty-Cycle Approach . . . . . . . . . . . . . . 45

3.5 Summary . . . . . . . . . . . . . . . . . 47

4 2D Preoperative RRT Path Planner for Steerable Bevel-Tip Needles in Multiple-Targets Soft Tissue $\quad 50$

4.1 Introduction . . . . . . . . . . . . . . . . . 50

4.2 Single-Target RRT-Based Path-Planning Algorithm . . . . . . . . . . 51

4.2.1 Rapidly Exploring Random Trees . . . . . . . . . . . . . . . . 52

4.2.2 Biasing the Algorithm . . . . . . . . . . . . . 53

4.3 Multiple-Target RRT-based Path-Planning Algorithm . . . . . . . . . 68

4.3.1 Clinical Relevance of the Algorithm . . . . . . . . . . . . . . . 69

4.3.2 Experimental Results . . . . . . . . . . . . . . 77 
4.4 Importance of the DC approach . . . . . . . . . . . . . . . . 82

4.5 Summary .............................. 83

5 3D Real-Time RRT Path Planner for Steerable Bevel-Tip Needles to Reach Multiple Moving Targets in a Simulated Real-World Ap$\begin{array}{ll}\text { plication } & 86\end{array}$

5.1 Introduction . . . . . . . . . . . . . . . 86

5.1 .1 Assumptions . . . . . . . . . . . . . . . . . 90

5.2 Single-Target Motion Planning . . . . . . . . . . . . . . . . . . . . 92

5.3 RRT-Based Intraoperative Motion Plan . . . . . . . . . . . . . . . . . 98

5.4 Intraoperative Multi-Target RRT-based Path-Planning Algorithm for Flexible Bevel-Tip Needle . . . . . . . . . . . . . . . . . . . . . . . . 99

5.5 Simulations and Discussion . . . . . . . . . . . . . . 102

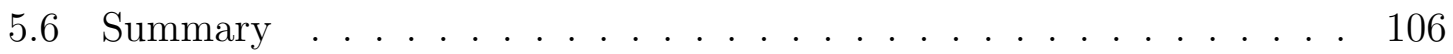

$\begin{array}{lll}6 & \text { Conclusions } & 107\end{array}$

6.1 Thesis Conclusion . . . . . . . . . . . . . . . . . 107

6.2 Possible Directions for Future Research . . . . . . . . . . . . . . . . . 108

7 Breadth-First Search (BFS) Algorithm [1] 111

8 Curvature Estimation and Online Adaptation 113

List of References $\quad 114$ 


\section{List of Tables}

4.1 Numerical analysis of the performance of the RRT-based planner for a single target of different locations and target regions under no algorithm biasing condition. The results correspond to $n=100$ simulations. 60

4.2 Numerical analysis of the performance of the RRT-based planner for different types of random sampling $\theta_{0}$, for each of which $\theta_{0}$ is set once during the algorithm execution. Two different target locations are considered for both of which $r=1 \mathrm{~mm}$ and $\kappa_{0}=0.2 \mathrm{~cm}^{-1}$. The results correspond to the mean values of $n=100$ simulations, where cost and time are in $\mathrm{cm}$ and $\mathrm{ms}$, respectively. . . . . . . . . . . . . . 62

4.3 Numerical analysis of the performance of the RRT-based planner for a single target of different locations under two different ways of biasing the algorithm. The results correspond to $n=500$ simulations. . . . .

4.4 Numerical analysis to compare the performance of our new multi-target RRT-based planner with that of the traditional planner for both the small and large workspaces. The results correspond to $n=100$ simulations. . . . . . . . . . . . . . . . . . 


\section{List of Figures}

1.1 Different levels of autonomy of a closed-loop needle steering system. a) Fully automated needle steering system, b) Semi-automated needle steering system. In both figures, using feedback information, the needle is navigated inside soft tissue to reach a target while avoiding any collision with obstacles. Needle navigation is done based on an online path-planning algorithm that enables the real-time control of needle motion inside soft tissue, adopted from [2] . . . . . . . . . . .

1.2 Effect of tip symmetry/asymmetry on needle path curvature during insertion into soft tissue. a) In symmetric-tip needles, the balanced tissue reaction force applied to the needle tip causes the needle to follow a straight path. b) In bevel-tip needles, the asymmetric force applied to the needle's bevel tip causes the needle to bend and follow a curved trajectory during its motion, adopted from [3] . . . . . . . . 
2.1 a) A 6-DOF parallel robot performs the spinal needle insertion through base steering, reprinted from [4] (with permission from the authors, D. Glozman and S. Moshe). b) Experimental setup for a needle insertion task in a real-time breast tumor biopsy. Linear actuators positioned along the $\mathrm{X}$-axis are used to steer the target toward the needle path (Y-axis) through phantom tissue manipulation, reprinted from [5] (C) 2009, IEEE). c) Experimental setup for controlling the bevel-tip needle trajectory inside the phantom tissue through tip steering. Two DC motors are used to insert and rotate the needle shaft during insertion, reprinted from $[6]$ (C 2015, IEEE) . . . . . . . . . . . .

2.2 a) Different types of tip asymmetry: (I) bevel, (II) pre-bend, (III) precurve, and (IV) kinked bevel, adopted from [7]. b) Flexure-tip needle design. The nitinol wires that comprise the flexure joint bend at the gap between the needle shaft and the tip as forces are applied to the bevel tip by tissue. c) Tissue damage comparison of a kinked bevel-tip needle, a clinical bevel-tip needle, and a flexure-base bevel-tip needle. Figures (b) and (c) are both reprinted from [8] (C 2013, IEEE). . .

2.3 a) A tissue phantom with markers painted on its top surface, reprinted from [9] with permission (C) Simon P. DiMaio, 2003). b) Interactive virtual needle insertion in a planar environment, reprinted from [10] (C) 2005, IEEE). c) Simulated needle intercept of a small virtual biopsy target embedded within elastic tissue. The needle is inserted into the side of a tissue model that is rigidly fixed along one edge. Due to tissue deformation, the needle fails to intercept the target, reprinted from [9] with permission (ㄷ Simon P. DiMaio, 2003). . . . . . . . . 18 
2.4 A graph (roadmap) is constructed once offline through sampling the free configuration space by nodes (empty circles) and providing the obstacle-free connections between nodes by edges (straight lines). The grey areas represent obstacles. The configurations $q_{\text {init }}$ and $q_{\text {goal }}$ are first connected to the roadmap through $q^{\prime}$ and $q^{\prime \prime}$. Then, a graphsearch algorithm returns the shortest path denoted by the thick black lines, reprinted from [11] (C) under Creative Commons Attribution 4.0 International license) . . . . . . . . . . . . . . . . . .

2.5 A tree is constructed online by sampling the free configuration space iteratively. The grey areas $X_{O b s}$ represent obstacles, while the green area $X_{\text {Goal }}$ refers to the goal region. (1-3) Relate to some intermediate iterations of the whole procedure until the RRT tree connects to $X_{\text {Goal }}$. (4) Using graph-search algorithm, the path connecting the initial configuration to $X_{\text {Goal }}$ is extracted from the tree, reprinted from [11] (C) under Creative Commons Attribution 4.0 International license). . . . . . .

2.6 The ultrasound transducer is robotically controlled to move synchronously with the needle tip. The needle tip pose is estimated using the 2D US image, needle insertion device, and the transducer positioning device. The estimated needle tip pose is used by the motion planner at each re-planning step to generate many feasible paths to the target. The planner selects the best path such that the needle path length is minimized while avoiding obstacles, reprinted from [12] (C) 2014, IEEE). 27

3.1 A robotic needle steering system, reprinted from [13] (C) SAGE Publications, 2014). . . . . . . . . . . . . . . . . . . . . . 34

3.2 Planar unicycle model, adopted from [14]. . . . . . . . . . . . 36

3.3 Spatial unicycle model. . . . . . . . . . . . . . . . . . . . . 38 
3.4 Different possibilities of the needle tip circular motion in the local coordinate frame $R F_{n}$ are shown in blue arcs of curvature $\kappa_{0}$. For the constant-curvature case, the reachability region belongs to the surface of the volume (blue arcs); however, using the DC approach, the extended reachability region includes the space inside the volume, too. A red curve is a representative path in the extended reachability region, adopted from $[15] . \ldots \ldots \ldots \ldots \ldots . \ldots . \ldots$

3.5 Experimentally obtained trajectories of a flexible bevel-tip needle at various duty cycles. (a) 0 duty cycle (no spinning). (b) 33 duty cycle. (c) 67 duty cycle. (d) 100 duty cycle (constant spinning), reprinted from [16] (c) 2007 IEEE). . . . . . . . . . . . . . . . . . 48

3.6 Simulated trajectories of a flexible bevel-tip needle at various duty cycles, reprinted from [16] (C) 2007 IEEE). . . . . . . . . . . . . .

4.1 Bevel-tip needle configuration in a plane. Parameters $P$ and $\theta$ represent the needle tip position and orientation, respectively. a) Relates to the bevel left direction, $\lambda=1$, where the needle tip follows a path of constant curvature $\kappa_{0}$ counterclockwise. b) By flipping the bevel direction to the right, $\lambda=-1$, the needle tip position does not change; however, by further inserting the needle, it follows the circular path with the same curvature but clockwise, adopted from [17]. . . . . .

4.2 Geometric representation of a piece-wise circular arc segment $C_{i}$ which is characterized by $\left(l_{i}, r_{i}, \phi_{i}\right)$. Given the needle tip configuration at $\mathrm{A}\left(X_{A}=\left[y_{A}, z_{A}, \theta_{A}\right]^{T}\right)$ and sampled point's position at $\mathrm{B}\left(P_{B}=\right.$ $\left.\left[y_{B}, z_{B}\right]^{T}\right)$, arc parameters can be uniquely obtained and used in the planner, adopted from $[18] \ldots \ldots \ldots$. . . . . . . . . 56 
4.3 Time duration $\Delta_{i}$ is split into four duty-cycled intervals $T_{i}$. Each $T_{i}$ is composed of two intervals: spinning interval $T_{\text {spin }_{i}}$ and non-spinning interval $T_{i n s_{i}}$, adopted from $[18] \ldots \ldots \ldots$. . . . . . . . . . . . . . . . .

4.4 Comparison of the performance of the RRT-based planner for two types of biasing the algorithm, i.e., bias 2 and bias 4 . The target is shown in black bullet $\left(T_{b o}\right) \ldots \ldots \ldots \ldots$

4.5 Multi-target planning includes individual RRT-based planning for each target. As a result, there are as many sequential insertions and retractions of the needle as the number of the targets. . . . . . . . .

4.6 Planner's efficiency in reaching targets $T_{3}$ and $T_{4}$, which are almost in the same direction of the needle bevel tip; after reaching target $T_{3}$, without necessarily having to retract, the needle is navigated toward $T_{4}$. 76

4.7 Multi-target planning under the novel RRT-based planner. The retraction states are tagged by small black bullets. The subscripts for both the targets and retraction states represent the order in which each is reached. . . . . . . . . . . . . . . . .

4.8 Comparison of our new multi-target RRT-based planner with the traditional one for the system configuration shown in Figs. 4.5 and 4.7. The cost and time data are normalized with respect to the corresponding mean value of the traditional planner. The results correspond to $n=100$ simulations. . . . . . . . . . . . . . . .

4.9 The planner is unable to visit all the 10 randomly-chosen targets $T_{i}, i=$ $1, \ldots, 10$. Regarding $T_{4}$, the planner fails in view of the nonholonomic constraints of needle motion and limited real clinical metrics. . . . .

4.10 The novel multi-target RRT-based planner for two sizes of workspaces: a) the smaller workspace, b) the larger workspace with doubled dimensions of the smaller one. . . . . . . . . . . . . . . . 
4.11 The ratios of the costs associated with the needle insertion procedure are compared for different values of $\kappa_{\min }$ for both the small and the large workspaces. The result for each value of $\kappa_{\text {min }}$ corresponds to 100 simulations. All the data are normalized with respect to the cost mean value of our new planner where $\kappa_{\min }=0 \ldots \ldots \ldots$

4.12 The ratios of the simulation run time are compared for different values of $\kappa_{\min }$ for both the small and the large workspaces. The result for each value of $\kappa_{\min }$ corresponds to 100 simulations. All the data are normalized with respect to the time mean value of our new planner where $\kappa_{\text {min }}=0$. To provide a clearer graph, the results for the marginal value of $\kappa_{\min }=.18$ are not presented. The ratios corresponding to $\kappa_{\min }=.18$ are 157 and 145 for the small and the large workspaces, respectively. This abrupt increase in the simulation run time reveals the noticeable degradation in the planner's efficiency while using almost constant-curvature trajectories. . . . . . . . . . . . .

5.1 Closed-loop configuration of a robotic needle steering system. The needle insertion device robotically inserts and rotates the needle base during intervals of $T_{\text {ins }}$ and $T_{\text {spin }}$ defined by the duty-cycled steering algorithm. The desired needle tip trajectory is computed by the RRTbased multi-target path-planning algorithm. The needle path curvature and the maximum curvature are both defined by the estimation algorithm. The needle tip pose is estimated online through the realtime ultrasound needle tracking system, adopted from [12] . . . . .

5.2 Geometric representation of a piece-wise circular arc segment $C_{i}$ which is characterized by $(l, r, \phi)$ where $\phi$ is the change of bevel direction, $l$ and $r$ are the arc length and radius, respectively, regenerated from [18]. 94 
5.3 Novel multi-target RRT-based motion planning for the steerable needle inside in-homogeneous tissue including moving targets and obstacles. The initial and the final locations of the targets are shown in blue dots and circles, respectively. Target indices show the order in which each target is reached. Moreover, the retraction states $r_{i}$ and $r_{i 2}$ in MT$\mathrm{RRT}_{3 \mathrm{D}}$ are mentioned in red and blue $\times$, respectively. The maximum and minimum targeting error is $1.023 \mathrm{~mm}, 0.388 \mathrm{~mm}$ respectively. . . 103

5.4 In-homogeneous properties of the tissue used in Fig. 5.3 . . . . . . . . 104

5.5 Evaluation of our new path planner for steerable needle motion inside in-homogeneous tissue in a simulated real-world application. a) targeting error per target and b) variation of the needle natural curvature during the path for each target. All results correspond to 50 simulations. 104

5.6 The performance of our new intraoperative RRT-based planner in a homogeneous tissue to reach five randomly chosen moving targets. a) The two left-sided plots show the targeting error of both our novel planner and the traditional one. b) The right-sided plot shows the cost ratio of our new planner vis-a-vis the traditional planner. All results correspond to 50 simulations. . . . . . . . . . . . . . 105

7.1 Demonstration of Breadth-First Search, regenerated from [1] . . . . . 112 


\section{List of Acronyms}

\begin{tabular}{ll} 
Acronyms & Definition \\
\hline \hline MIS & Minimally Invasive Surgery \\
RRT & Rapidly-exploring Random Tree \\
PRM & Probabilistic Road Map \\
PF & Potential Field \\
MSM & Mass Spring Model \\
FEM & Finite Element Method \\
HC & Hunt Crossly \\
KV & Kelvin Voigt \\
DC & Duty Cycle \\
SMR & Stochastic Motion Roadmap \\
DP & Dynamic Programming \\
DOF & Degree Of Freedom \\
RF & Reference Frame
\end{tabular}




$\begin{array}{ll}\text { SO } & \text { Special Orthogonal } \\ \text { SE } & \text { Spatial Euclidean } \\ \text { BFS } & \text { Breadth First Search } \\ \text { ST } & \text { Single Target } \\ \text { MT } & \text { Multiple Target } \\ \text { IRRT } & \text { Intra-Operative Rapidly-Exploring Random Tree } \\ \text { SVD } & \text { Singular Value Decomposition } \\ \text { PCA } & \text { Principle Component Analysis } \\ \text { LSA } & \text { Least Squares Algorithm }\end{array}$




\section{List of Symbols}

\begin{tabular}{ll}
\hline Acronyms & Definition \\
\hline \hline$v$ & needle insertion speed \\
$\omega$ & needle rotation speed \\
$r$ & radius of needle's circular path \\
$\kappa$ & curvature of needle's circular path \\
$\kappa_{0}$ & needle's natural curvature \\
$\phi$ & change of needle's bevel orientation \\
$R F_{w}$ & fixed world reference frame \\
$R F_{n}$ & moving reference frame attached to needle tip \\
$X$ & notation matrix of needle tip \\
& \\
& needle tip pose
\end{tabular}




\begin{tabular}{|c|c|}
\hline so $(3)$ & lie algebra of $\mathrm{SO}(3)$ \\
\hline$S E(3)$ & special Euclidean group of 3 \\
\hline$s e(3)$ & lie algebra of $\mathrm{SE}(3)$ \\
\hline$\wedge, \vee$ & isomorphic operators \\
\hline$v$ & linear velocity vector \\
\hline$\omega$ & rotational velocity vector \\
\hline$u_{1}, u_{2}$ & two control inputs \\
\hline$t$ & time \\
\hline$n(t)$ & needle tip position as a function of time \\
\hline$\alpha / D$ & duty cycle factor \\
\hline$T$ & duty cycling period \\
\hline$T_{\text {spin }}$ & spinning time interval per $T$ \\
\hline$T_{\text {ins }}$ & non-spinning time interval per $T$ \\
\hline$h$ & a function that represents needle path curvature \\
\hline$\theta$ & change of needle's tip orientation \\
\hline$\lambda$ & planar bevel direction parameter \\
\hline$X$ & needle tip pose \\
\hline$P_{\text {rand }}$ & random position \\
\hline$C$ & circular arc segment \\
\hline
\end{tabular}




\begin{tabular}{|c|c|}
\hline$l$ & arc length \\
\hline$P_{\text {goal }}$ & target position \\
\hline$P_{o b s t}$ & obstacle position \\
\hline$K$ & maximum number of iterations \\
\hline $\mathfrak{P}$ & needle tip path \\
\hline $\mathfrak{T}$ & RRT tree \\
\hline$Q_{\text {reach }}$ & reachable set from $P_{\text {rand }}$ \\
\hline$X_{\text {near }}$ & closest state to $P_{\text {rand }}$ in $Q_{\text {reach }}$ \\
\hline$X_{\text {new }}$ & potential new state \\
\hline$\rho$ & distance metric \\
\hline$J$ & cost function \\
\hline$J_{i}$ & cost of needle insertion and retraction \\
\hline$J_{o}$ & cost of obstacle collision \\
\hline$d_{o}$ & needle tip distance from the closest obstacle \\
\hline$\alpha_{i}, \alpha_{o}$ & scaling factors of $J_{i}$ and $J_{0}$ \\
\hline$S_{T}$ & set of targets \\
\hline$S_{r}$ & set of retraction states \\
\hline$r_{i}, r_{i 2}$ & retraction state \\
\hline$Q_{t, \text { reach }}$ & collision-free reachable set from $r_{i}$ \\
\hline
\end{tabular}




\begin{tabular}{ll}
$q_{t, \text { near }}$ & closest target to $r_{i}$ in $Q_{t, \text { reach }}$ \\
$Q_{t, 2 \text { reach }}$ & collision-free reachable set from $q_{t, \text { near }}$ \\
$e_{1}, e_{2}, e_{3}$ & standard basis of $\Re^{3}$ \\
$\Delta$ & replanning time interval \\
$X_{\text {nest }}$ & estimated needle tip pose at the end of each $\Delta$ \\
$X_{0 \text { mes }}$ & measured needle tip pose at the beginning of each $\Delta$ \\
$\phi_{\text {model }}$ & $\phi$ parameter of the model \\
\hline
\end{tabular}




\section{Chapter 1}

\section{Introduction}

\subsection{Motivation}

Needle insertion procedures have been gaining in popularity with medical communities over recent years due to their less invasive nature, resulting in decreased recovery time, cost, trauma, and complications compared to traditional methods [19]. Currently, their applications span a wide range of diagnostic and therapeutic procedures including biopsy, ablation, brachytherapy for cancer treatment, neurosurgery, drug injection and there is still a growing tendency toward integrating needle insertion into other minimally invasive surgeries (MIS) and procedures. Needles can also be used in virtual training systems with the aim of increasing the safety of the needle insertion

procedure [20]. In each one of them, the efficiency of the procedure highly depends on accurately localizing the needle tip towards a predefined location inside soft tissue while ensuring the safety of the procedure as well. For example, in brachytherapy, misplacement of implanted radioactive seeds can damage the healthy tissue. Also, an error in final needle tip positioning during biopsy can yield misleading information.

The ultimate goal in a needle insertion system is to control the needle navigation during insertion within soft tissue in order to reach a target or a set of targets while avoiding any collision with any potential obstacle with as little tissue trauma 
as possible. Manual needle insertions have such limitations during the procedure as the surgeon's limited precision, limited tool maneuverability, needle deflection and tissue deformation, imperfect imaging feedback, and the difficulty of performing for the surgeon. The latter one is due to the under-actuated characteristics of needle motion inside soft tissue. As a result, it is not always feasible to accurately perform needle insertion procedures manually. Consequently, for accurate needle steering inside soft tissue, more explicit methods are needed. It is little wonder, then, that the automation or semi-automation of manual insertions in the hope of raising the success rate has gained in popularity owing to higher accuracy, fewer needle insertions, faster and safer performance [21]. Fig. 1.1 shows the block diagrams of two different levels of autonomy in a closed-loop needle steering system based on online motion planning. Fig. 1.1a refers to a fully automated system, wherein both needle insertion and rotation are done automatically, while Fig. 1.1b refers to a semi-autonomous needle steering system, wherein the surgeon is in the loop. The surgeon is in charge of needle insertion using a robotic handheld instrument, while the instrument rotates the needle automatically. Unlike these two methods, in manual needle insertion, needle steering is done thoroughly by the surgeon based on visual and haptic feedback. As explained in [2], regarding the trade-off between safety, accuracy, and clinical implementation for different levels of automation granted to the needle steering system, manual needle steering systems are less accurate vis-a-vis semi- and fully-automated needle steering systems. Moreover, they offer higher levels of patient safety and are clinically more compatible than their semi- and fully-automated counterparts.

The current practice of needle insertion procedures is based on using straight rigid needles in operating rooms to reach specific targets inside soft tissue. This is because these needles are easier to control and the path is well defined. However, given the special anatomy of the human body, in some applications of needle insertion procedures, it is not possible to reach a target in a straight line. Rather, a steerable 

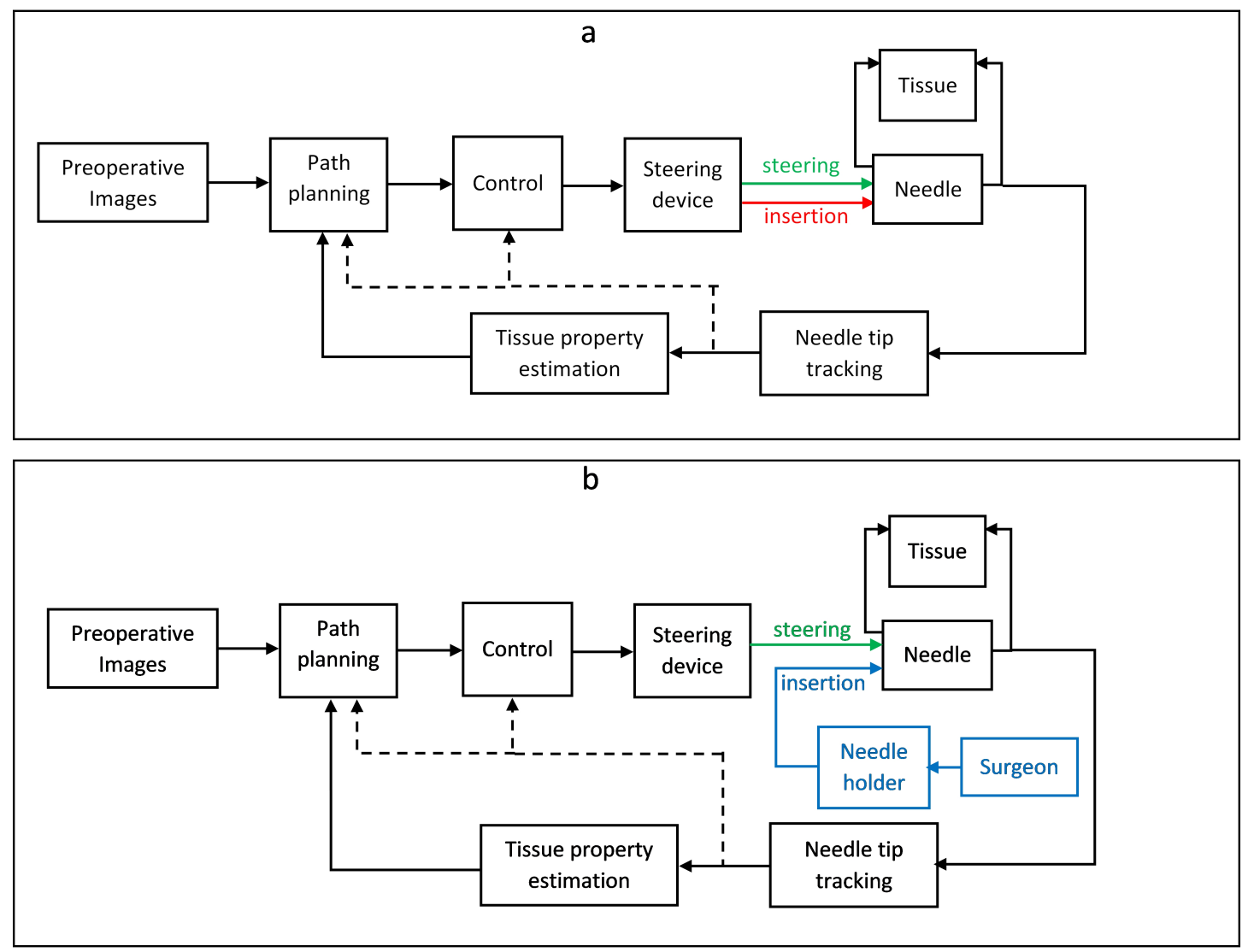

Figure 1.1: Different levels of autonomy of a closed-loop needle steering system. a) Fully automated needle steering system, b) Semi-automated needle steering system. In both figures, using feedback information, the needle is navigated inside soft tissue to reach a target while avoiding any collision with obstacles. Needle navigation is done based on an online path-planning algorithm that enables the real-time control of needle motion inside soft tissue, adopted from $[2]$.

needle is required to be actuated accurately to follow a more complex trajectory so that the needle does not collide with impenetrable objects such as bones and damage any sensitive tissue structures such as nerves or arteries. It bears noting that the main problem with controlling these needles is the cost associated with the improved mobility of steerable asymmetric-tip needle's motion. The advantage of a bevel-tip flexible needle over a rigid needle has been shown in [22]. Consequently, the concept 
of motion planning and control of steerable bevel-tip needles has garnered significant research interest in robotic needle steering, particularly, whenever any collision with impenetrable objects or sensitive tissue structures has to be avoided [23].

\subsection{Multiple-Target Path Planning}

The majority of the works introduced in the literature address the path-planning problem for a single target. On the other hand, multi-target path planning has a wide range of applications. In prostate brachytherapy, the needle has to visit multiple locations within the prostate gland to implant the radioactive seeds in predefined locations in order to eradicate the cancerous tissue. Moreover, in liver biopsy, multiple samples of the liver organ have to be examined. The main challenge facing a single target path-planning problem is increasing the needle's maneuverability during its motion while simultaneously ensuring less tissue trauma during the procedure as much as possible. However, when it comes to a path-planning problem with multiple targets, the main challenge relates to the amount of tissue trauma caused during needle insertion, rather than the needle's accurate steering. For example, in a prostate brachytherapy procedure, which is one of the major applications of needle steering, several radioactive seeds have to be implanted by the needle inside the prostate gland to eradicate the cancerous tissue. According to the current trend in operating rooms, which is based on manual needle placement, the needle has to be sequentially inserted to and completely retracted from each separate target. This method inevitably decreases the efficiency of the procedure and causes intensive tissue traumas. As stated in [3], the literature shows that reducing the surgical traumas of the prostate can help lessen the severity of edema and, consequently, improve implant dosimetry and reduce toxicity. Therefore, more explicit methods, which are less invasive, have to be examined during multiple-target needle insertion procedures. In this regard, through 
robotic needle steering, it is possible to obviate the need for an individual insertion to and complete retraction from each separate target, which in turn results in surgical trauma reduction. Our study addresses the robotic multiple-target path-planning problem for steerable needles.

\subsection{Robotic Flexible Bevel-tip Needle Steering}

In current manual needle insertion procedures, needle insertion is usually done by the surgeon through manipulating the tissue which requires considerable training and experience on the part of the surgeon. Based on the under-actuated characteristic of steerable needle motion inside soft tissue and the different inherent complications such as limited visibility, difficulty of needle maneuverability, needle deflection and tissue deformation arise during needle insertion, it is virtually impossible for the surgeon to manually provide an accurate control over the needle motion in the face of all these complications. Incorporating robots into manual insertions could help to overcome the inherent limitations of a manual needle insertion. Some studies focus on the use of robots along with surgeons, rather than the full automation of the procedure. However, due to the significant potential of robotic needle steering systems in the control and path planning of steerable needles within soft tissue, robotic flexible needle steering is frequently used in current research practices. As opposed to straight rigid needles, steerable needles have a great degree of maneuverability during their motion inside soft tissue. This property is a particular boon given the extreme significance of avoiding any collision with any potential obstacle. In particular, our interest is in flexible bevel-tip needles. In [24], the effect of the needle diameter and the tip type on the needle path curvature is explained. It shows that beveltip needles tend to bend more easily than the symmetric conic and triangular tip needles. Also bevel-tip needles are more sensitive to tissue variations, while the other 
two yield a more robust behavior. More importantly, bevel-tip needles deliver higher maneuverability over obstacles and sensitive tissue structures. Henceforth, whenever we refer to a needle insertion system in our work, we mean a robotic flexible bevel-tip needle insertion system.

\subsubsection{Nonholonomic Kinematics of Bevel-Tip Needle}

As explained in [14], while a flexible bevel-tip needle is pushed against soft tissue, due to tip asymmetry of the needle, the unbalanced reaction forces are applied at the needle-tissue interface respectively. This leads the needle to bend in the direction of the bevel as it is pushed into the tissue. This is illustrated in Fig. 1.2. As shown, the symmetric needle tip follows a straight line when it is inserted within soft tissue. On the other hand, path maneuverability can be achieved through asymmetric needle tips.

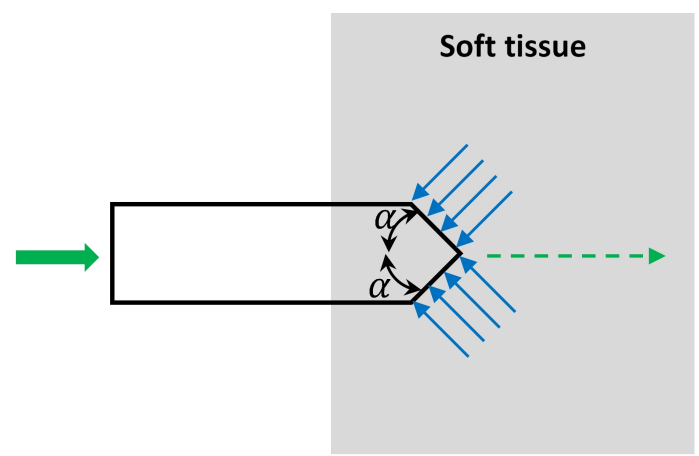

(a) Symmetric needle tip

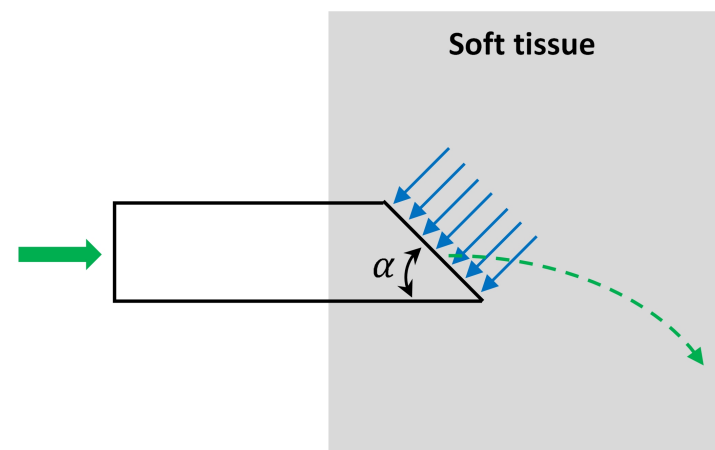

(b) Asymmetric (Bevel) needle tip

Figure 1.2: Effect of tip symmetry/asymmetry on needle path curvature during insertion into soft tissue. a) In symmetric-tip needles, the balanced tissue reaction force applied to the needle tip causes the needle to follow a straight path. b) In bevel-tip needles, the asymmetric force applied to the needle's bevel tip causes the needle to bend and follow a curved trajectory during its motion, adopted from [3].

Webster et al. in [14] experimentally validated that the bevel-tip needle can be 
modeled as a nonholonomic system with a steering constraint. Similar to unicycles and bicycles, there are certain directions in which the bevel tip cannot instantaneously move. Likewise, the bevel tip can reach any desired pose in the plane of interest through a more complex sequence of motions including needle insertion, needle retraction to a certain distance, reorientation of the bevel tip, and reinsertion inside the soft tissue. As steerable bevel-tip needles offer higher maneuverability compared to rigid needles, it is possible to reach previously inaccessible targets while at the same time avoiding collision with any sensitive or impenetrable objects. Therefore, bevel-tip needles are of high interest in needle insertion procedures and their path planning is an active research topic.

\subsubsection{Randomized Path-Planning Algorithms}

For the purpose of path planning of flexible bevel-tip needle, different approaches have been introduced in the literature. However, in recent years, sampling-based approaches have become far more popular in path planning problems [25]. Without having to explicitly characterize the configuration space, the time complexity of these randomized algorithms decreases significantly [26]. As explained in [27], among the randomized path-planning methods, the Rapidly-Exploring-Random-Tree (RRT) approach is a better candidate for a broad class of path-planning problems comparing to Probabilistic-Roadmap (PRM) [28] and randomized Potential-field (PF) [29] methods. Although both of the latter two randomized approaches (PRM and PF) are applicable in high-dimensional configuration spaces, the randomized potential filed method highly depends on the choice of a good heuristic potential function which in case of obstacle avoidance problem for systems with differential kinematic constraints proves a daunting task. Regarding the PRM, a graph is constructed based on the randomly chosen configurations and subsequent connection of the neighboring pairs of configurations through a local planner. In general, in nonholonomic systems, 
the connection problem could be difficult as designing a nonlinear controller, consequently for a graph with as many numbers of states, the path-planning problem may prove an involved task. On the other hand, RRT algorithms are well designed to handle nonholonomic constraints and are a proper candidate for systems with high dimensional configuration space. As explained in [11], the computational complexity of these algorithms is $\mathcal{O}(\log n)$, where $n$ is the number of samples. An RRT is incrementally expanded through necessary control inputs to drive the system towards randomly chosen points in the free configuration space. This process continues until the target is reached. Ultimately, the path connecting the target to the needle entry point is extracted from the tree. Since high dimensional configuration space and nonholonomic constrains are both the characteristics of a flexible bevel-tip needle motion within soft tissue, RRT methods are frequently adopted for the path-planning task of flexible bevel-tip needles inside soft tissue.

\subsubsection{Preoperative vs. Intraoperative Path Planning}

In a generic needle insertion procedure, different properties affect the performance of the procedure. In regard to the needle, its diameter, tip type/shape, flexibility, rigidity, and torsional compliance affect the performance. Regarding the tissue, its elasticity, linearity/non-linearity, homogeneity/heterogeneity, isotropy/anisotropy, and patient-specific properties such as age, gender, mass, temperature, etc. are important factors that can affect the procedure significantly. However, compared to needle-related properties, tissue-related properties are more difficult to quantify and less certain due to the inherent inevitable variations associated with a needle insertion procedure. Consequently, a great amount of variations and uncertainties surrounds needle-tissue interaction, making it almost impossible to obtain a comprehensive model that can capture all aspects of a real-world needle-tissue interaction. Also, as explained in [30], working with real specimens of soft tissue for experimental 
studies leads to different complications. Apart from ethical concerns and specimen scarcity, the data obtained is often unreliable or inconsistent due to the complex needle-tissue interaction. Thus, tissue phantoms are widely used as a surrogate for real tissue in different research practices. Tissue phantoms are easier to work with and do not represent sample-specific variations, enabling clinicians to practice medical procedures in a more controlled environment. Besides, it provides a better platform for new findings in the research area. While using phantoms, it is reasonable to express the respective needle-tissue interaction with a nominal model with a limited number of parameters. Particularly, one popular model that our work is based on is a single-parameter model [14]. Because of these facts, a great number of the works introduced in the literature are based on tissue phantoms that address the preoperative path-planning problem of steerable needles, which are based on deterministic models with fewer parameters. Drawing on tissue phantoms, preoperative path-planning approaches are good candidates when it comes to discovering promising directions in the realm of path planning for steerable needles. Thus, Chapter 4 focuses on the preoperative path planning of steerable bevel-tip needles. Yet, it is clear that the limited-parameter models, including the one-parameter model that our work is based on, cannot reflect the difficulties associated with the needle-tissue interaction in biological soft tissue including tissue deformation, needle deflection, tissue inhomogeneity, patient variability, and a great amount of uncertainty involved in real-world applications. Consequently, for better assessing the feasibility and performance of any proposed approach to clinical implementation, in-vivo tests have to be carried out, the focus of Chapter 5, where we will introduce the intraoperative path planning and control of bevel-tip needle motion within biological soft tissue in simulated real-world applications. 


\subsection{Contributions}

There has been a great deal of attention and studies on the path-planning problem of steerable needles, and many researchers are still working hard to either make an improvement to the already introduced works or introduce new methods. Due to the current improvements in medical imaging modalities and computer programming and analysis, most of the past burdens have been cleared away. However, it seems much more research still has to be done and the concerns have to be addressed more adequately before applying the introduced technologies into operating rooms. A great majority of the introduced works address the path-planning problem of a flexible needle insertion system in a single-target platform. On the other hand, undoubtedly, multi-target path-planning problem has important applications such as prostate brachytherapy for cancer treatment. In the context of multi-target path-planning problem for flexible bevel-tip needles various works have been carried out. However, most of them address one of the issues including the preoperative path-planning problem, path-planning problem under certain limitations, or path-planning problem without a proper clinical relevance. The limited number of targets, obstacle-free environments, and planar motion instead of spatial motion are some of the limitations associated with the path-planning problems. To the best of the our knowledge, intraoperative path planning of steerable needle inside soft tissue to reach multiple targets in a real-world application has never been presented anywhere in the literature before. Another salient feature of our planner is its practicality in terms of clinical metrics by focusing on more clinically relevant needle-tissue interaction parameters. Unlike global optimization-based methods with exponential time complexity to the number of targets and system complexity, our planner performs well independently of these factors. Finally, as the need for individual insertion and complete retraction of the needle for each target is obviated, the total tissue damage decreases significantly. The 
following publications are based on this thesis.

$\mathbf{J}_{\mathbf{1}}$ A. N. Aghdam and P. X. Liu, "Some Issues on Needle Insertion Procedures: A Review," Instrumentation, DOI: 10.15878/j.cnki.instrumentation.2019.02.009, vol.6, no.2, pp.71-89, Jun. 2019

$\mathbf{J}_{2}$ A. N. Aghdam and P. X. Liu, "A Novel Path Planner for Steerable Bevel-Tip Needles to Reach Multiple Targets with Obstacles," in IEEE Transactions on Instrumentation and Measurement, April 2020.

$\mathbf{J}_{3}$ A. N. Aghdam and P. X. Liu, "A Novel Real-Time Path Planner for a Steerable Needle to Reach Multiple Moving Targets in 3D Dynamic Environments in a Real-World Application," Journal paper in preparation.

\subsection{Thesis Organization}

The rest of the thesis is structured as follows. In Chapter 2, we present a literature review of steerable bevel-tip needle motion, path planning, and control inside soft tissue. In Chapter 3, steerable bevel-tip needle motion kinematics and differential kinematic constraints are discussed. In this regard, both the planar and the spatial kinematic models and constraints are presented. In Chapter 4, we introduce our new planar preoperative multi-target planner for the steerable bevel-tip needle. In Chapter 5, we develop our novel 3D multi-target planner for a simulated real-world application, where tissue and obstacles may move due to tissue deformation. Tissue variability is accounted for in our planner, too. Finally, Chapter 6 summarizes the thesis and proposes some potential directions for future research in the field of multitarget path planning for steerable needles. 


\section{Chapter 2}

\section{Related Work}

\section{$2.1 \quad$ Introduction}

Needle insertion procedures have dramatically increased in popularity in medical applications in recent years. Its less invasive nature, which is performed locally on the body, largely explains this growing trend, and consequently results in less intraoperative tissue damage and shorter post-operative recovery time. Many procedures like biopsy, deep brain stimulation, and cancer treatments are done using needles/catheters. However, despite all the advantages of needle insertion procedures, the inherent complications resulting from them, such as tissue deformation, needle deflection, tissue inhomogeneity, patient variability, and the inherent uncertainty in needle-tissue interaction, can hardly be missed. Therefore, a needle insertion procedure requires that we address promising aspects and associated concerns. In this chapter, we provide a literature review of the achievements made in percutaneous needle insertion procedures to date. In particular, our review highlights the pathplanning and control problem of steerable bevel-tip needles during insertion within soft tissue. 


\subsection{Modeling Needle-Tissue Interaction}

A rather comprehensive review of needle insertion into soft tissue can be found in [31]. In manual needle insertion procedures, it is not always feasible to perform a satisfactory procedure by the surgeon due to implicit associative complications. On the other hand, robotic or robotic-assisted surgery is becoming more and more popular among medical communities due to the amazing achievements made in robotics, automation, control, and visual-based measurement. Today, the robotic needle-based procedure is an extremely important issue in research practice, and researchers are making every attempt to increase the success rate of needle steering inside soft tissue. In [32], a robotic needle insertion system with active needle steering was developed. Their experimental setup provides stereo imaging feedback as well as controlled actuation for needle linear and rotation motions. Furthermore, needle buckling was avoided using a telescopic support sheath. The goal is to improve the performance of a needle insertion system by enhancing needle steerability while moving around obstacles. In [2] and [33], a review on issues associated with closed-loop robotic needle insertion systems is presented.

Previous robotic needle insertion systems including [34-36] have addressed reducing the targeting error through the initial alignment of the needle prior to insertion. The robotic system drives the stainless rigid needle along a straight trajectory. These systems are not capable of accounting for the needle bending during insertion. On the other hand and in recent years, steerable needle robotic systems which are less dependent on perfect initial alignment of the needle are become popular due to the ability of reducing targeting error as well as providing control on needle bending during insertion. As explained in [3], robotic needle steering based on needle bending can be achieved through a variety of mechanisms, including base steering (lateral manipulation) [37], [4], tip steering [38], and applying forces to the tissue during 
insertion [5], [39].

In the base-steering approach, using a symmetric-tip needle which is made of stainless steel, and is stiff relative to the tissue, steering is achieved through pulling on and angling the needle shaft outside the body. The method of steering via deforming the tissue can generate greater steering capability at shallow depth but this ability degrades as insertion depth increases. Therefore, in order to provide the same level of steering, a greater amount of tissue has to be deformed through applying increased force. Since there is a safety threshold on the applied force on the tissue before it is torn, this method is only applicable to certain depths. In contrast to base steering, in the tip steering method, an steering capability almost independent of the insertion depth can be achieved through an asymmetric bevel-tip needle that is flexible relative to the tissue. Steering is generated through an unbalanced tissue reaction force applied on the needle's bevel tip. In the latter approach [5] and [39], instead of manipulating the needle to reach a target inside soft tissue, tissue manipulation is performed through a robotic system to pull the target into the path of the needle and at the same time push the obstacles out of the path of the needle. Fig. 2.1 shows the robotic needle insertion systems that provide different types of steering. Enhanced steerability can be extended to pre-bent and pre-curved needles with higher potential for maneuverability comparing to bevel-tip needles. Fig. 2.2a shows different types of needle tip asymmetry including bevel tip, pre-bend (kinked) tip, pre-curved tip, and kinked bevel tip. Thomas in [7] showed that in a pre-curved needle, the tip path curvature is inversely correlated to the arc length of the pre-curved section. Apart from its increased steerability, there are some negative implications associated with pre-curved needles. Specifically, due to the increased stress imposed on tissue, lateral tissue deformation increases, and the greater discrepancy between the needle tip path and the needle shaft is seen. With kinked bevel-tip needles, even higher curvature can be achieved, but with increased tissue damage. This is because of 


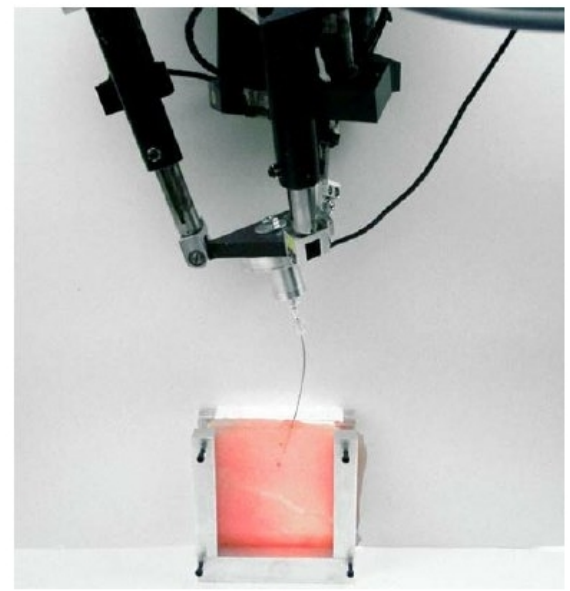

(a)

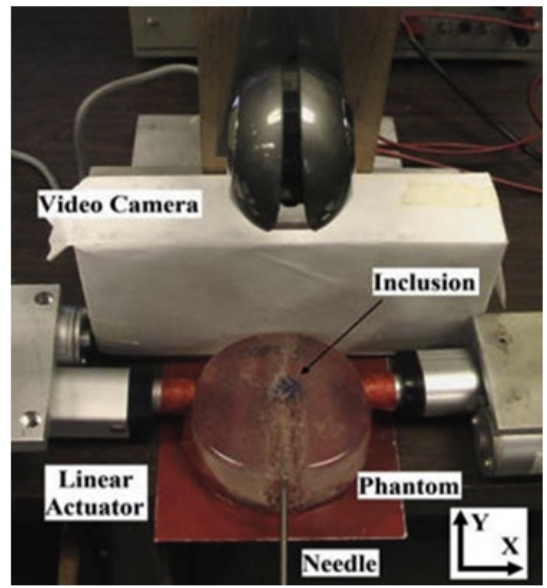

(b)

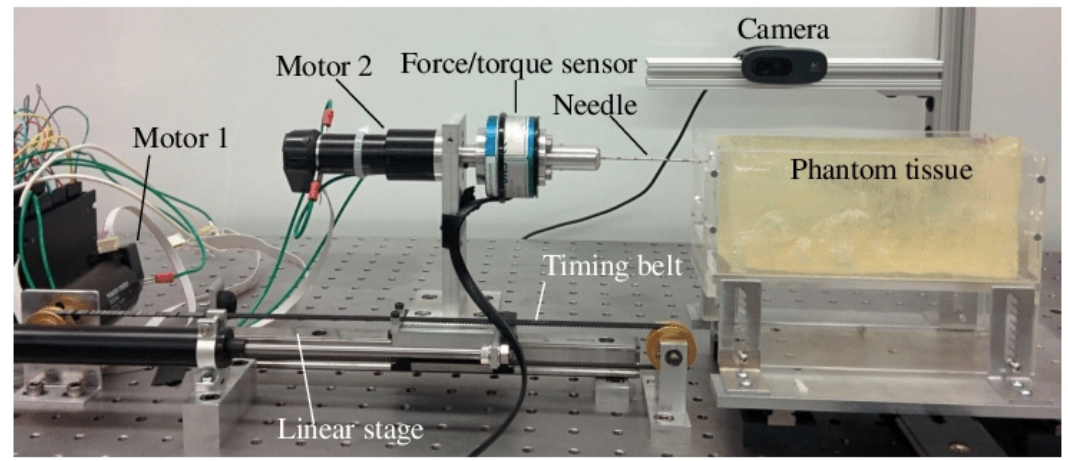

(c)

Figure 2.1: a) A 6-DOF parallel robot performs the spinal needle insertion through base steering, reprinted from [4] (with permission from the authors, D. Glozman and S. Moshe). b) Experimental setup for a needle insertion task in a real-time breast tumor biopsy. Linear actuators positioned along the $\mathrm{X}$-axis are used to steer the target toward the needle path (Y-axis) through phantom tissue manipulation, reprinted from [5] (c) 2009, IEEE). c) Experimental setup for controlling the bevel-tip needle trajectory inside the phantom tissue through tip steering. Two DC motors are used to insert and rotate the needle shaft during insertion, reprinted from [6] (C) 2015, IEEE).

the local helical path generated during the axial rotation of the needle base. This issue is addressed in [8] and solved by introducing flexure-based bevel-tip needles, which offer both high steerability similar to kinked bevel-tip needles and less tissue damage simultaneously. The bevel tip kinks during pure insertion in response to 
tissue reaction forces. Moreover, during axial rotation the flexure tends to bend the kinked bevel tip back to its initial straight configuration. Fig. 2.2b shows a flexure-based bevel-tip needle and Fig. 2.2c reveals the reduced tissue damage during insertion with a flexure-based bevel-tip needle compared to a kinked bevel-tip needle [8]. The main step in analytically analyzing the needle-tissue interaction is to obtain

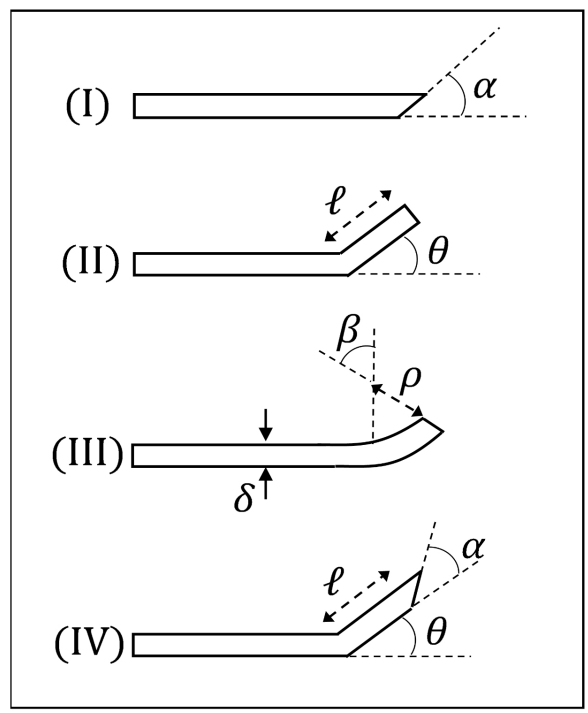

(a)

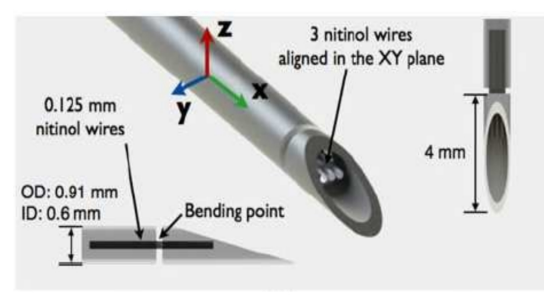

(b)

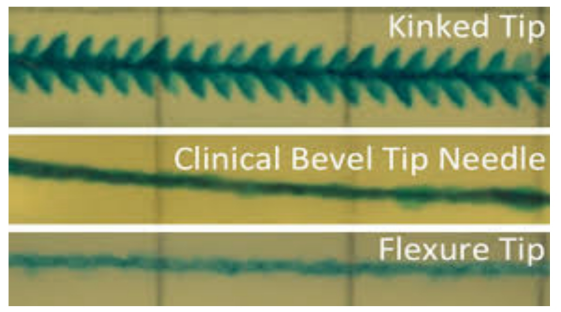

(c)

Figure 2.2: a) Different types of tip asymmetry: (I) bevel, (II) pre-bend, (III) precurve, and (IV) kinked bevel, adopted from [7]. b) Flexure-tip needle design. The nitinol wires that comprise the flexure joint bend at the gap between the needle shaft and the tip as forces are applied to the bevel tip by tissue. c) Tissue damage comparison of a kinked bevel-tip needle, a clinical bevel-tip needle, and a flexure-base bevel-tip needle. Figures (b) and (c) are both reprinted from [8] (c) 2013, IEEE).

a kinematic model of the needle tip motion inside soft tissue. Different models have been introduced in the literature to mathematically represent needle kinematics and dynamics during insertion inside soft tissue. A review on modeling of needle-tissue interaction can be found in [40].

Two common methods used for soft tissue modeling are the finite-element model 
(FEM) and the mass-spring-damper model (MSM). FEM is based on continuum mechanics focusing on precision, while MSM calculates discrete deformations at a higher numerical rate. DiMaio [37], for the first time, used the FE model to consider elastic tissue deformation caused by a relatively stiff needle during insertion. By placing markers on the tissue phantom surface, as shown in Fig. 2.3a, he experimentally monitored the planar nodal tissue mesh deformations. To ease the burden of numerical calculation, he assumed a quasi-static linear elastic tissue model. As a result, the tissue nodal forces were estimated by solving a set of linear equations. He used this estimated force distribution to simulate a virtual planar, shown in Fig. 2.3c, which helped surgeons and medical residents to improve their experience by virtually rehearsing various aspects of a needle insertion. Also, using the condensation technique and low-rank matrix updates, he improved computational efficiency. To achieve a more realistic model, he extended his previous work to [10], considering a slip-stick model in the needle-tissue contact interaction. His work also accounted for needle flexion and orientation changes by considering a large strain elastic model for needle. Moreover, varying velocity and nonconforming needle trajectories are considered. Fig. 2.3b shows the respective interactive virtual needle insertion in a planar environment based on the measured planar tissue deformations and needle insertion forces. Although FEM for tissue material has significantly contributed to modeling and simulation, its real-time application is limited to small linear deformations. For larger deformations, the nonlinear stress component has to be considered instead, and subsequent expensive numerical analysis has to be carried out. In [41], using a 2D dynamic FEM, a thin, rigid needle insertion is simulated during manual prostate brachytherapy. Barbé et al. in [42] proposed a method for the online robust estimation of needle interaction forces for in-vivo/in-vitro experiments based on a variant of recursive least squares algorithm. They broke down the insertion process into three phases: needle insertion before puncture, needle penetration inside tissue, and needle 


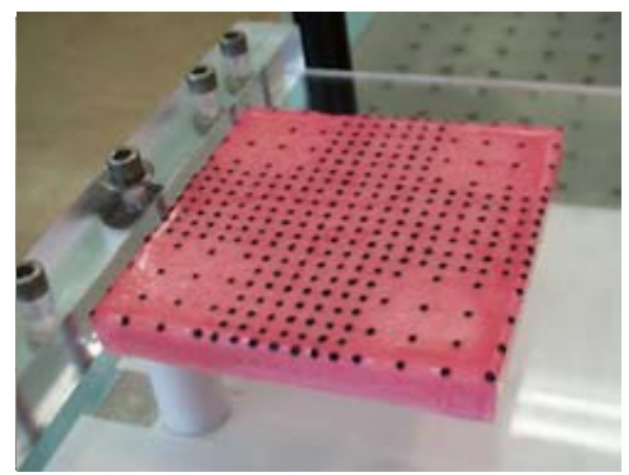

(a)
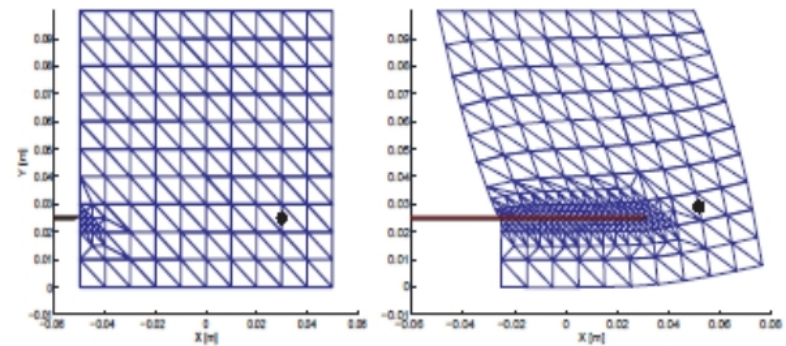

(c)

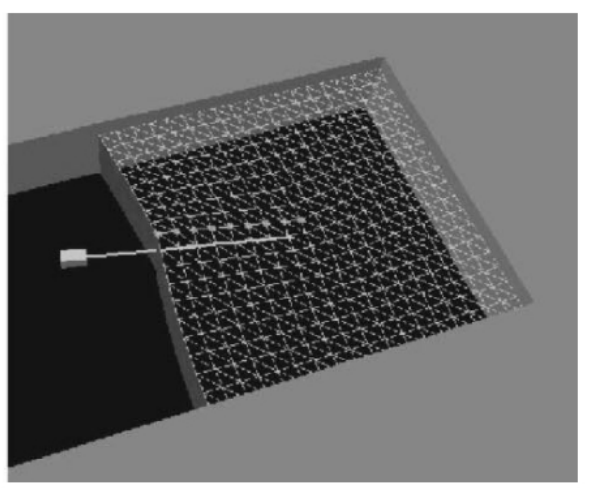

(b)

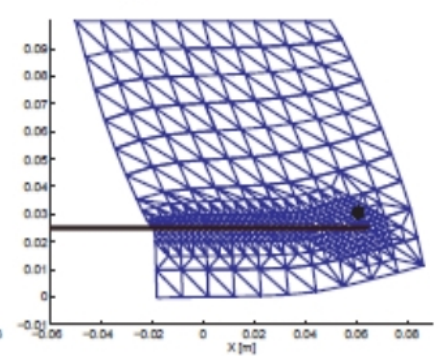

Figure 2.3: a) A tissue phantom with markers painted on its top surface, reprinted from [9] with permission (c) Simon P. DiMaio, 2003). b) Interactive virtual needle insertion in a planar environment, reprinted from [10] (C) 2005, IEEE). c) Simulated needle intercept of a small virtual biopsy target embedded within elastic tissue. The needle is inserted into the side of a tissue model that is rigidly fixed along one edge. Due to tissue deformation, the needle fails to intercept the target, reprinted from [9] with permission (C) Simon P. DiMaio, 2003).

retraction. The available measured data was needle tip position and velocity. Since the velocity was obtained by numerical differentiation of position data, Kalman filter was used to address the associated perturbations caused by quantization error. The authors compared two distinct models for the first phase, i.e. the Kelvin Voigt model and the Hunt Crossly model. HC model's generality in incorporating nonlinear interaction associated with larger deformations is its competitive edge over the KV model. During insertion, they modeled the insertion forces as a summation of cutting, friction and persisting tissue compression, the latter of which was not considered in [43]. 
Since only the position of the needle tip, which is completely different from the position of the needle entry point on the surface, is available, separation of different force components during insertion without fiducials is not feasible. For practical purposes, they modeled the needle insertion force by simply generalizing the KV model to the time varying version in which model parameters, $\mathrm{K}$ and $\mathrm{B}$ (stiffness and damping), depend on the needle tip position and velocity.

Unlike [37] and [10], which estimate the force distribution along the needle shaft nodes; Okamura [43] modeled the needle driving forces as a combination of three components: capsule stiffness, tissue cutting, and friction forces. The first component refers to the pre-puncture phase and was modeled by a nonlinear spring, while the other two refer to needle penetration inside soft tissue and were modeled by a constant parameter and a modified Karnopp friction model, respectively. The model parameters are then derived experimentally through proper curve fitting functions during ex-vivo tests.

As opposed to [37], [10] where steerability of the stainless steel needle is achieved through noticeable soft tissue deformations caused by manipulating the needle base, Webster et al. [14] modeled a highly flexible bevel-tip needle insertion in a relatively stiff material, which carried negligible tissue deformations, consequently inflicting less damage on the surrounding tissue. They developed 2D unicycle and bicycle models for steering the bevel-tip needle inside soft tissue. Furthermore, using the Lie group theory, the 3D generalization of unicycle and bicycle models is presented. If the needle-tissue pair is chosen correctly, in which the tissue is stiff relative to the needle, the curvature of the needle path is considered constant and tissue deformation is neglected. Moreover, needle-tissue friction and torsional compliance of the flexible needle are not taken into account in their model.

Since both translational and rotational speeds of the needle motion inside soft tissue are usually of low rate, studies often focus on needle motion kinematics and 
ignore dynamics by assuming that the needle is in an equilibrium state during each time step. However, in practice, insertion velocity does matter and can be used as a control degree of freedom. In this regard, some studies have looked at needle insertion dynamics. Drawing on fracture-based mechanics, Mahvash in [44] studied the dynamics of needle insertion into soft tissue with particular focus on transitions between different tissue layers. The fact that ruptures are developed with uncontrolled crack extension could lead to unexpected behavior of the needle tip with a negative influence on tip position accuracy. He also spotted an inverse correlation in that as the insertion velocity increases, both the rupture force and deformation decreases, and, as a result, less tissue damage and more controlled behavior is experienced during rupture. As insertion velocity cannot be raised beyond a certain point for safety and instability considerations, he obtained an optimal velocity for practical application. In a similar vein, Khadem et al. [24] studied the mechanics of inhomogeneous soft tissue cutting and broke down the whole insertion process into three phases similar to [43], with negligible tissue resistance/reaction forces. Tissue cutting forces, which are considered constant in [43], is reviewed analytically as a function of needle tip geometry, tissue mechanical properties, and insertion velocity. Khadem et al. in [45] derived the detailed dynamic equations of robotically assisted needle insertion into soft tissue and predicted both the needle shape and the tip position while considering tissue resistance forces. They proposed a coupled two-body rigid/flexible model for the needle carriage/shaft. The flexible part is modeled by a compliant beam, which undergoes tissue resistance forces as the external force. Their study, like many other mechanics-based modeling, is constrained to planar analysis.

From an experimental perspective; in view of current measurement limitations and model parameter identification challenges, researchers prefer using synthetic tissue phantoms with more consistent properties rather than using living tissue in their 
experimental studies. This is because tissue phantoms provide a translucent, homogeneous, and more controlled environment for repeated needle insertions. However, as discussed in [30], tissue phantoms do not mimic the complete behavior of real tissue and as a result, they are not perfect substitutes for real tissues. Therefore, for real-world clinical implementation, in-vivo tests have to be carried out. In addition to [30], Wedlick in [46] carried out a comparative study of needle insertion in artificial phantoms and ex-vivo tissues using a robotic needle insertion system. He experimentally observed that the common artificial phantoms do not closely mimic the ex-vivo behavior and exhibit different stiffness, friction, and relaxation properties. Furthermore, their study revealed that the torque measured during needle rotation is related to the needle shaft force measured during insertion. A similar trend is observed for the recorded motions. Based on their work, it is possible to obtain a needle-tissue interaction model without having to advance the needle through the skin, which leads to a less destructive model identification process.

\subsection{Needle Steering, Control, and Path Planning}

In many medical applications, it is desirable to control needle navigation inside the body to reach a target or a set of targets while avoiding collision with any potential obstacle. In the simplest form of needle steering, the goal is to steer the needle in such a way that it follows a straight line. Therefore, needle deflection has to be modeled and, then, compensated for. Some relevant works can be found in [47-50]. It is not always feasible to reach a target through a straight-line path. In fact, in most cases, the needle is required to provide some degree of maneuverability during its motion to avoid collision with any potential obstacle. This further brings to the fore the importance of the path-planning concept in a needle steering system. 
Regarding the compromise between optimality and time complexity in pathplanning methods, the optimization-based approaches [51-53] suffer from the timeconsuming feature; this problem worsens as the problem dimensionality increases. Consequently, restricts their application to open-loop planning. On the contrary, sampling-based approaches have become far more popular in the field of path planning. Without having to explicitly characterize the configuration space, the time complexity of these randomized algorithms decreases significantly. Two famous approaches are PRM [54] for multi-query path-planning problems and RRT [27] for single-query path-planning problems, shown in Fig. 2.4 and Fig. 2.5, respectively.

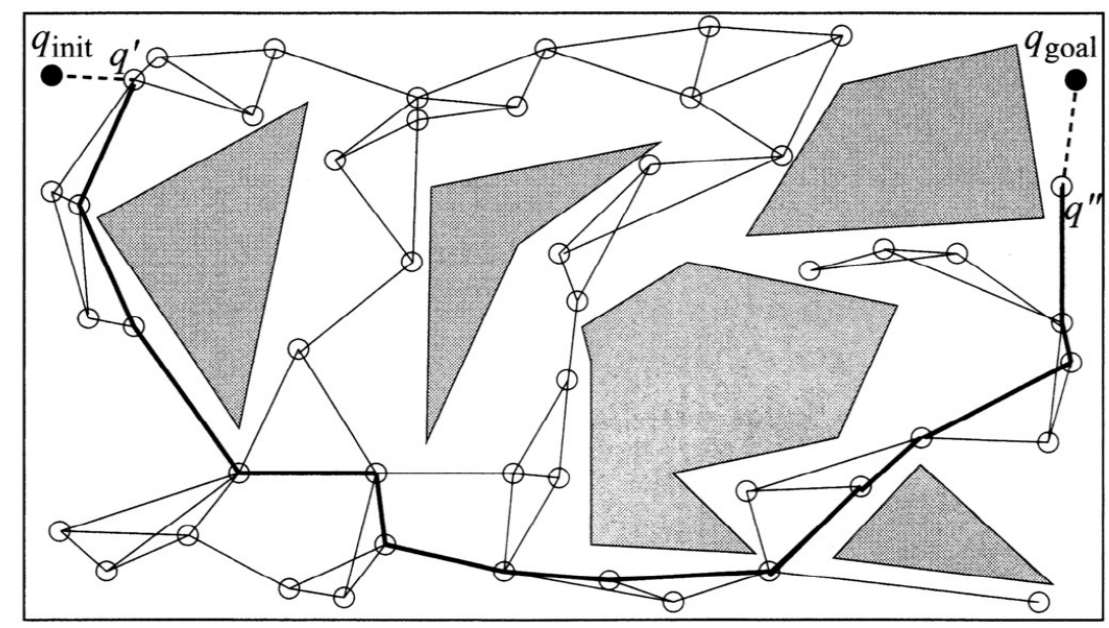

Figure 2.4: A graph (roadmap) is constructed once offline through sampling the free configuration space by nodes (empty circles) and providing the obstacle-free connections between nodes by edges (straight lines). The grey areas represent obstacles. The configurations $q_{\text {init }}$ and $q_{\text {goal }}$ are first connected to the roadmap through $q^{\prime}$ and $q^{\prime \prime}$. Then, a graph-search algorithm returns the shortest path denoted by the thick black lines, reprinted from [11] (ㄷ) under Creative Commons Attribution 4.0 International license).

Other modifications of the original algorithms are available in the literature such 

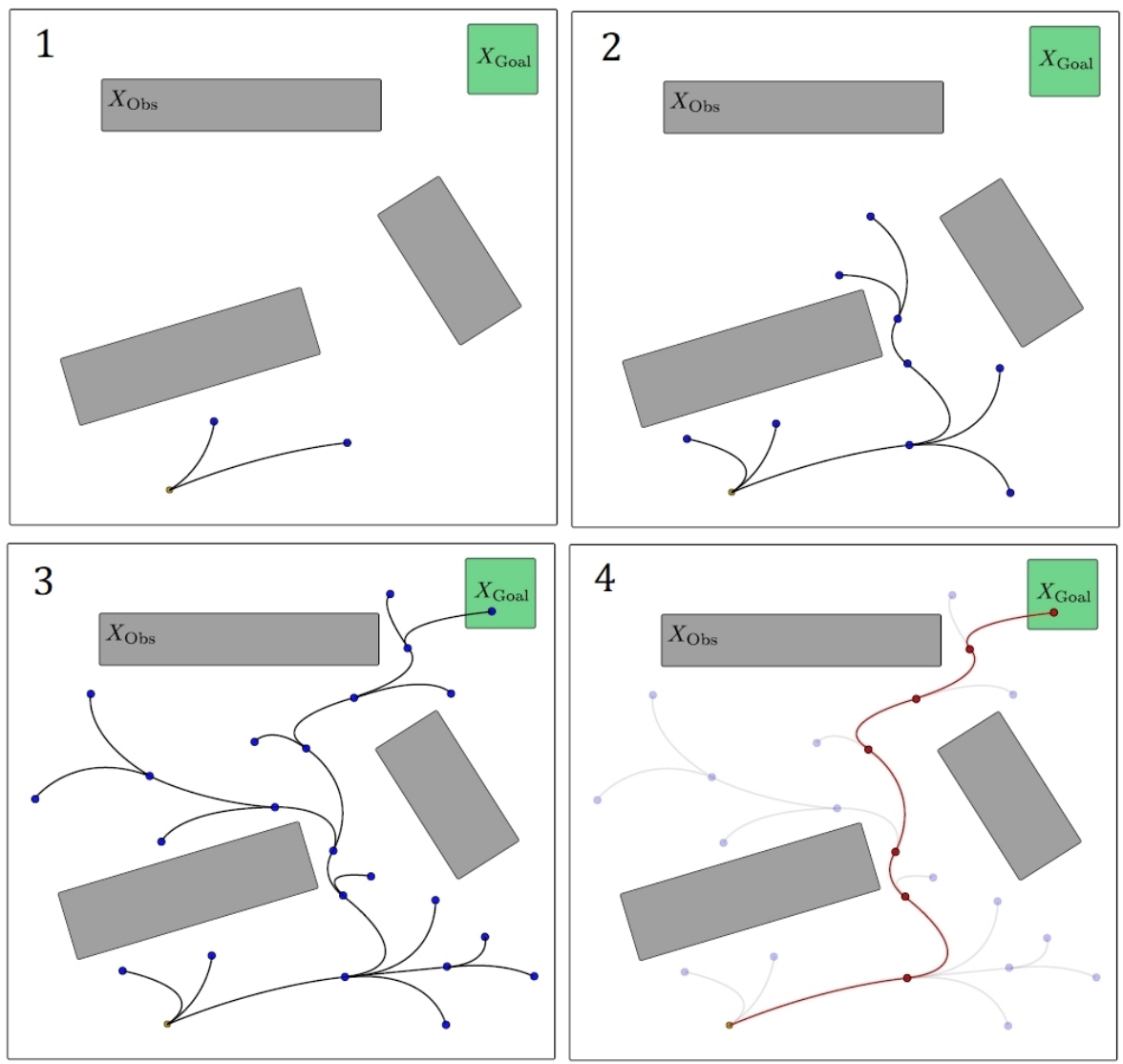

Figure 2.5: A tree is constructed online by sampling the free configuration space iteratively. The grey areas $X_{O b s}$ represent obstacles, while the green area $X_{\text {Goal }}$ refers to the goal region. (1-3) Relate to some intermediate iterations of the whole procedure until the RRT tree connects to $X_{\text {Goal }}$. (4) Using graph-search algorithm, the path connecting the initial configuration to $X_{\text {Goal }}$ is extracted from the tree, reprinted from [11] (C) under Creative Commons Attribution 4.0 International license).

as [55] introduced by Alterovitz et al. The authors in [55] developed a RapidlyExploring Roadmap (RRM) planning algorithm for a single-query optimal pathplanning problem. Their approach provides a user-specific balance of exploration versus refinement. 


\subsubsection{Single Target}

\section{Preoperative Planning}

In [51], a 2D planner uses extensive numerical optimization to find the locally optimum path for steerable needle inside deformable soft tissue with the presence of obstacles. The effect of soft tissue deformation is considered using finite element simulations. In [52], a screw-based motion planning for 3D environments with obstacles is developed through discretization of the control space rather than the configuration space. They introduced two different discretization strategies, namely stop-and-turn and helical strategies. Both methods result in a locally optimal path computed in less than a few seconds. In a different work, DiMaio in [53] developed a 2D potential-fieldbased path-planning approach for flexible symmetric-tip needles during insertion in deformable soft tissue in the presence of obstacles. The planner uses a needle manipulation Jacobian to relate base steering to tip velocities. Tissue deformation, needle flexibility, and contact behavior are also accounted for in the model. The scope of this work is limited to preoperative planning. In [56], Duindam et. al. introduced a shortdistance 3D path planning approach for steerable needles using inverse kinematics. The fast geometric-based planner originally computes a feasible path for obstacle-free environments. However, there is some possibility of both avoiding obstacles and obtaining a better solution among certain possible candidates using the concept of null motions. Like [52], they used a stop-and-turn strategy with the limitation of eight consecutive turnings and insertions. However, their planner provides no completeness guarantee. In [57], two RRT, one rooted at the origin and one at the target, grow simultaneously to explore the configuration space around them as well as toward each other using a greedy heuristic. Since it is possible that there is no feasible path found for given initial and target configurations, in [58], Xu et. al. addressed the problem of finding a feasible entry point for a given target and initial region. This is solved 
using RRT with back-chaining where the tree explores the 3D configuration space with obstacles backward from the given target configuration toward the specified initial region. They also introduced a new exploring strategy whereby the new state is added to the tree by random sampling of the control space rather than deterministic sampling [58]. This could ease the burden of computation considerably.

Although PRM planners are capable of addressing changes in target motion, since the roadmap is once computed preoperatively, they are not capable of handling obstacle motion and other sources of motion uncertainties. Similarly, Stochastic Motion Roadmap (SMR)-based methods, introduced by Alterovitz et al. in [59], [60] to address the issue of uncertainty in planar needle motion, are not proper candidates for intraoperative applications. Using SMR, the problem is formulated as a Markov Decision Process (MDP) and is solved through DP to return a stochastically optimal path. However, the computationally expensive feature of these methods limits their applicability to preoperative methods.

\section{Intraoperative Planning}

In any steerable needle path-planning approach, the objective is to predict the needle trajectory based on steering commands, and subsequently, by integrating the needle steering system into the needle tip tracking system, the intraoperative control of the needle trajectory can be achieved. Glozman and Shoahm in [61] developed a robotic system for steering a flexible needle under real-time fluoroscopic guidance. Using an inverse kinematics algorithm, based on a virtual spring model, they calculated the required base manipulation so that the needle tip follows the planned trajectory. A similar work with updated tissue stiffness parameters has been done in [62] under real-time ultrasound imaging. Sachin in [15] developed an RRT planning algorithm for steerable needles in complex 3D environments including obstacles and narrow passages at an interactive rate. Using 3D real-time imaging feedback, 
it is possible to edit the environment in real time by adding or changing obstacles. Instead of using a classic RRT algorithm, which tries to find the best control input to expand the tree and which is quite computationally expensive, they encoded the segments of the needle trajectory based on geometric quantities instead of control inputs and calculated the feasible geometric trajectory composed of piece-wise circular arcs with different curvatures. Afterward, this geometric trajectory is mapped onto its equivalent motion plan based on a duty-cycling (DC) approach introduced in [16]. Using reachability-guided sampling heuristic [63], the planner efficiently applies the nonholonomic kinematic constraints of needle motion. In a similar vein, Bernardes et al. in [64] and [65] introduced an adaptive 2D path planning algorithm using duty cycling. In [64], they presented an arc-based RRT planner to intraoperatively compensate for motion uncertainties and disturbances. Also in [65], instead of point-sampling, they used an input-sampling version of a 2D RRT planner to achieve even faster computation of the needle trajectory for intraoperative use. They used an adaptive motion planner to continuously update the needle path. However, the 2D scenario used in the simulations considers the target region excessively large. In [18], Sachin et al. introduced a needle steering algorithm in $3 \mathrm{D}$ via rapid RRT replanning. Their planner and controller are implemented in a closed-loop configuration and are capable of dealing with real-world uncertainties in needle motion due to tissue deformation, needle deflection, and imperfect model parameters. Their method is similar to [15], but with a modified distance metric. Instead of choosing Euclidean distance to find the closest node in the tree, the arc length of each sub-trajectory is chosen as a better candidate. They also relaxed the constant-curvature constraint using the DC approach to performing variable-curvature trajectory planning. In addition, needle buckling is accounted in their planner. They empirically and in an offline fashion obtained the relationship between the duty-cycle and needle curvature by fitting a polynomial function to the recorded data of the needle tip pose at the 
end of each duty cycling interval throughout its motion. A similar work has been done by Vrooijink et al. in [13]. They performed 3D RRT re-planning and steered the steerable needle in a non-static environment using the 2D ultrasound images. Their approach accounts for real-world uncertainties in needle motion as well as both target and obstacle motion. Moreover, Moreira et al. in [12] worked on the same context of adaptive 3D RRT-based path planning for dynamic environments without prior knowledge of needle curvature, which is estimated on-line through ultrasound images. The introduced intraoperative methods [13], [15], [12, 18,64,65] attempt to regularly adapt the planned motion based on the most recent information obtained from the closed-loop feedback. Fig. 2.6 shows the closed-loop configuration of the integrated system introduced in [12]. These methods do not directly model uncertainty.

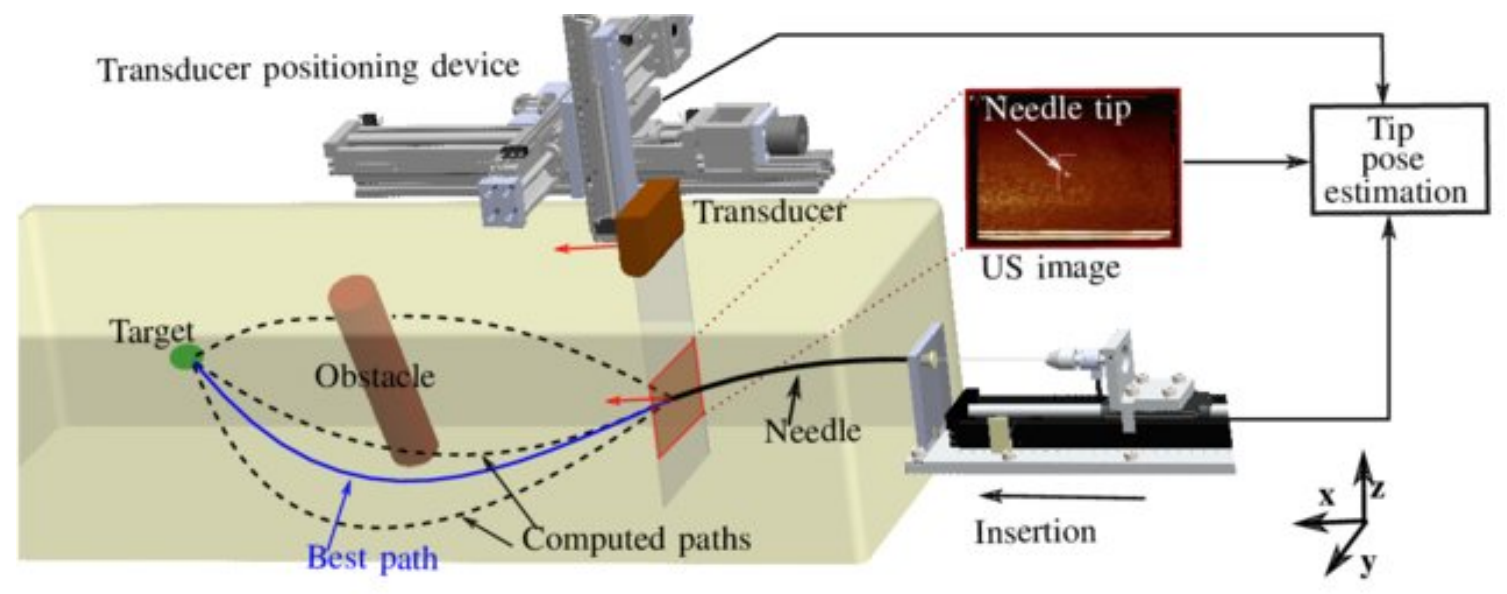

Figure 2.6: The ultrasound transducer is robotically controlled to move synchronously with the needle tip. The needle tip pose is estimated using the 2D US image, needle insertion device, and the transducer positioning device. The estimated needle tip pose is used by the motion planner at each re-planning step to generate many feasible paths to the target. The planner selects the best path such that the needle path length is minimized while avoiding obstacles, reprinted from [12] (C) 2014, IEEE). 
Instead, using deterministic models and monitoring the system response, uncertainties seen in the output are compensated. However, some shortest path model-based plans may lose efficiency and guide the needle through narrow corridors, which are less robust in the face of uncertainties and, consequently, may collide with obstacles. As a result, researchers started working on a different direction of accounting for uncertainties in needle motion based on the idea of explicitly modeling uncertainties in needle motion. The objective is to maximize the probability of success in a needle insertion procedure in terms of hitting the target without colliding with any obstacle. Alterovitz et al. in [60] first addressed the issue of uncertainty for planar needle motion. They sampled both the needle tip positions and the orientations and formulated the path-planning problem as a Markov Decision Process (MDP). They obtained the optimal sequence of discrete controls using Infinite Horizon Dynamic Programming (DP), while minimizing a user-specified cost function. Alterovitz et al. later in [59] introduced another sampling-based motion planning approach that explicitly considers uncertainty in needle motion in order to maximize the probability of success in terms of obstacle avoidance and target hitting. A Stochastic Motion Roadmap (SMR) is built similar to PRM by sampling collision-free states in a $2 \mathrm{D}$ configuration space. The collision-free connectivity of states is determined using distributions instead of binary values like PRM. Using SMR, the motions are locally sampled at each state for each control input and, then, the associated state transition probabilities are estimated. The problem is formulated as an MDP and is solved through DP to return a stochastically optimal path. A complete work of a 3D needle steering system that integrates an SMR planner [59] with a planar controller [66] to stabilize the needle in the desired plane with torsion compensation [21] has been developed by Reed et al. in [23]. Also, Alterovitz et al. in [67], based on a similar approach and just using the parameters extracted from the medical images, obtained intraoperative steering of needle motion inside soft tissue. Since SMR planners are 
based on a precomputed DP lookup table, the instantaneous closed-loop control of needle tip motion is possible only for static environments. Although Sovizi et al., in [68], relaxed the computational cost of Markov-Chain approach by simplifying the DP problem to LP formulation for intraoperative feedback control of flexible needles, their work is neither capable of addressing the obstacle and target motion nor multiple targets.

The above-mentioned control strategies require a limited number of model parameters in each of them. For an accurate control of the needle motion, the effect of model uncertainties has to be taken into account. In an attempt to remove the dependency of a method on a specific patient, or even on a certain organ, non-modelbased approaches have been proposed for needle steering and control. Bita et al. in [69] proposed a simple 2D image-guided sliding-based control algorithm to steer a bevel-tip needle toward a fixed location inside the tissue during brachytherapy. The scope of her work was a regulation problem where the needle has to move in a straight line or reach the desired deflection at the desired insertion depth in an obstacle-free environment. Out-of-plane deviations of the needle tip have been left out in their work. The needle tip deflection error, obtained from ultrasound imaging feedback, and its derivative are fed into the controller as inputs. Their method does not require any knowledge of needle or tissue characterizations except a rough estimate of needle tip curvature. Moreover, the algorithm is not sensitive to uncertainty in the needle curvature. During the needle's in-plane motion, the bevel tip orientation is discrete, either $0^{\circ}$ or $180^{\circ}$, and is characterized by the sign of curvature. Regarding the sliding-based controller, keeping the sliding surface as close as possible to zero is equivalent to maintaining zero error dynamics. A bevel flip is executed by the controller whenever the sign of the switching surface changes. This approach is in keeping with the increased number of bevel flips which can result in increased tissue trauma. To mitigate this observation, an alternative switching pattern with added 
hysteresis to the model is used. A higher choice of the switching threshold leads to a lower number of bevel rotations pointing to a trade-off between the targeting error and the number of bevel flips. The stability analysis and error convergence are carried out using the unicycle kinematic model and the Lyapunov stability method. Also, the 3D extension of this work with the presence of obstacle has been done by the same author in [70].

\subsubsection{Multi-Target Planning}

The majority of the works introduced in the literature address either the preoperative [51], [52], [59], [60] or intraoperative [15], [13,18,64,65] path-planning problem for a single target. Yet, the multi-target problem remains largely under-researched. Lee in [71] developed a 2D path planner for steerable needles with a single entry point through partial retraction, rotation, and reinsertion of the needle. They used the constant-curvature model [14] for needle motion inside tissue as well as the geometric relationship between multiple tangent circles. They found an optimal trajectory through expensive calculations. However, the scope of their work was limited to a maximum number of three targets and to obstacle-free environments. The extension of the geometric-based path planning to 3D environments is developed in [72] with similar assumptions. However, they discussed the extension to cases of more than three targets with obstacle avoidance in their paper. Lobaton et al. in [73] developed a constant-curvature path-planning algorithm for 2D environments with obstacles using PRM to reach multiple targets. Instead of point sampling as it is common in traditional PRM, they sampled circles of constant curvature and then generated the feasible transitions between them to construct the roadmap. They solved the multi-goal optimal planning problem using minimum directed Steiner tree over the roadmap. Using two heuristics, namely the shortest path first and the longest path first, they reduced the time complexity of the multi-target planning algorithm but 
at the same time lost optimality of the solution. Although they sacrificed optimality in favor of reaching lower time complexity, they obtained the solution within $\% 5$ of the global optimal solution. However, the assumptions they made in the simulations about the size of the workspace seems to be larger than what is often observed in real experiments with needles of more clinically relevant parameters.

$\mathrm{Xu}$ et al. in [74] introduced the extension of classic RRT planning to multiple needle insertions. They planned inserting multiple needles from a single small region to navigate steerable needles in 3D environments to reach multiple targets in an openloop fashion. Instead of growing every single tree independently and then connecting each goal to the entry region using constant-curvature sub-trajectories [14], a forest of rapidly exploring random trees, called fireworks trajectories, attempts to expand all trees simultaneously toward the entry region. Each of the trees is rooted at a specified target and using a back-chaining approach [58] the fireworks trajectories connects all targets to the needle entry region. However, inserting multiple needles simultaneously causes almost as much tissue damage as the sequential insertion of needles. Moreover, the interactions of different needles have not been considered in their work. [75] 


\section{Chapter 3}

\section{Needle Motion Kinematics through Soft Tissue}

\subsection{Introduction}

Modeling needle-tissue interaction is a key step in the simulation, control, and path planning of needle steering inside soft tissue in a robotic platform [3]. Different models have been introduced in the literature to mathematically represent needle kinematics and dynamics during insertion inside soft tissue. A comprehensive model that can fully capture the detailed features of needle-tissue interaction has yet to be developed. A review on modeling of needle-tissue interaction can be found in [40].

Modeling living tissue material and needle-tissue interaction forces are extremely challenging due to the inherent physiological and bio-mechanical variations of soft tissue before and during needle insertion. Even for a certain organ in a certain person, there are implicit uncertainties in soft tissue modeling due to periodic motions of breathing and heart beating, variation in body temperature and blood pressure, healthy versus sick tissue, etc. Moreover, soft tissue properties vary during insertion due to tissue inhomogeneity, nonlinearity, and anisotropy. Some of the introduced models consider more complex aspects of needle-tissue interaction during insertion 
inside soft tissue including needle's torsional characteristics, tissue deformations, and needle-tissue frictional behavior. Instead of explicitly considering these hard-to-model behaviors, which could be an involved task, alternative practical approaches have been introduced in the literature. Unlike needle insertion studies in [37] and [10], which are based on using symmetric-tip stainless steel needles with notable tissue deformations during needle insertion, Webster et al. in [14] used a highly flexible bevel-tip needle in a relatively stiff material. They experimentally observed that the needle tip trajectory looks like the final needle shaft shape. The data is obtained via a robotic system with two degrees of freedom: insertion and rotation about the insertion axis. In bevel-tip needles, steerability is mainly achieved through an unbalanced force distribution at the bevel tip. Hence, needle tip motion can be controlled by the axial rotation of its base. In view of the nonholonomic nature of bevel-tip needle kinematics in soft tissue, they developed 2D unicycle and bicycle models for steering the bevel tip needle inside soft tissue. Furthermore, using the Lie group theory, they extended their model to 3D spaces.

Chapter 2 reviewed research on needle insertion, steering, and control. This chapter introduces the flexible bevel-tip needle motion kinematics during insertion inside soft tissue. First, Section 3.2 looks at the necessary assumptions made in our work. Section 3.3, then, focuses on the mathematical expression of the 2D planar case, followed by the 3D generalization. More importantly, Section 3.4 introduces the variable-curvature needle motion kinematics through the Duty-Cycle approach. Finally, a summary of the chapter is presented in Section 3.5.

\subsection{Considerations and assumptions}

Webster et al. [32] experimentally verified that the constant-curvature motion of steerable bevel-tip needles is independent of needle insertion velocity for the correct 
choice of a needle-tissue pair, in which the tissue is stiff relative to the flexible beveltip needle. According to [32], we focus on needle motion kinematics and ignore the effect of needle dynamics in our work. Similarly, our work is based on the robotic needle insertion system using flexible bevel-tip needles to cause less tissue damage during insertion compared to traditional stiff needles. Fig. 3.1 shows the robotic

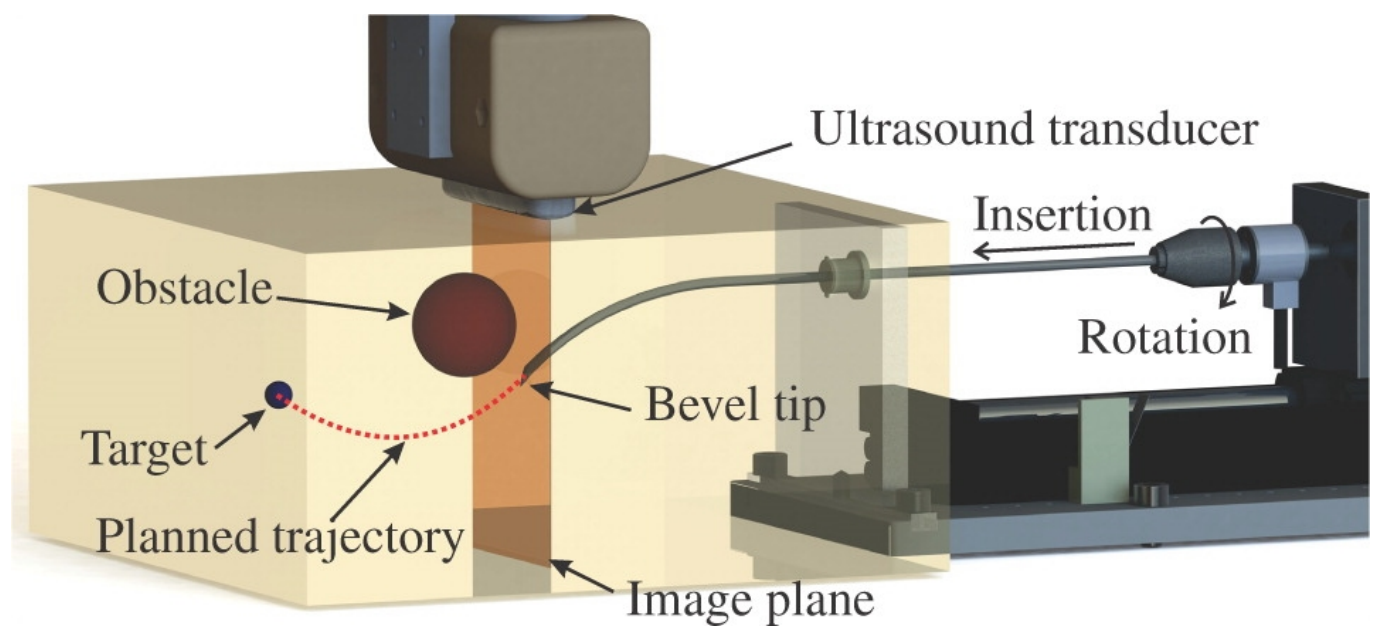

Figure 3.1: A robotic needle steering system, reprinted from [13] (C) SAGE Publications, 2014).

needle steering system used in [13], which has two control inputs: the insertion and rotation about the insertion axis, both actuated robotically at the needle base. As seen, a flexible bevel-tip needle is steered inside the soft-tissue phantom to reach the target while avoiding the obstacle. The closed-loop control is achieved through the imaging feedback obtained by the 2D ultrasound transducer. As explained in [14], by purely inserting the bevel-tip needle within the tissue, the tissue reaction force imposed on the bevel surface causes the needle tip to follow a circular path in the direction of the bevel, which is unchanged. By merely rotating the needle about its shaft while the tip position is unchanged, the bevel direction is reoriented which in turn reorients the plane containing future needle tip motion. Conjointly, the needle 
deflects along a curved trajectory in the direction of the bevel tip.

As explained in [14], the model is based on a few assumptions as follows.

- They assumed that the tissue is stiff relative to the needle through proper choice of needle material. Thus, the tissue is not displaced during needle motion. In other words, they ignored the macroscopic tissue deformation caused by the needle in their model.

- They ignored needle torsional compliance in their model and assumed that the needle stays in place through the surrounding tissue during rotation. As a result, any change in needle orientation at the base is transmitted perfectly to the needle bevel tip while the needle shaft stays in place.

Due to the above-mentioned assumptions, the needle shaft follows the path of the needle tip during its motion inside soft tissue. Also, the control inputs applied at the needle base are completely transmitted to the needle tip. Consequently, the needle motion is entirely characterized by its tip motion and controlled by two degrees of freedom (DOF), i.e., the insertion speed $v$ and the rotation speed $\omega$.

\subsection{Unicycle Model for Bevel-Tip Needle Motion}

Alike bicycles and unicycles, bevel-tip needles cannot move instantaneously in certain directions. Similarly, a bevel-tip needle can reach any desired pose in the configuration space through a combination of insertion, retraction, reorientation of the bevel tip, and reinsertion. Webster et al. in [14] developed a nonholonomic kinematics for flexible bevel-tip needle motion within tissue which is used frequently in control and path planning of these needles. The authors in [14] proposed a twoparameter bicycle and a one-parameter unicycle models for needle tip motion inside soft tissue. Since the needle path within the tissue is simply a single circular arc, 
the second parameter is inefficient and the needle motion can be sufficiently captured using a single-parameter unicycle model.

\subsubsection{Planar Needle Motion and nonholonomic constraints}

Fig. 3.2 shows the bevel-tip needle before and after changing the bevel orientation. In each case, a single wheel is located at the needle tip and a body reference frame $B$ is attached to the center of the wheel. In the standard unicycle planar model, the nonholonomic system has two independent control inputs (linear velocity $v_{z} \triangleq v$ and angular velocity $\omega_{x} \triangleq \omega$ are independent) and a single no-slip constraint $\left(v_{y}=0\right)$. However, in the bevel-tip needle motion, since the bevel angle is fixed, by applying

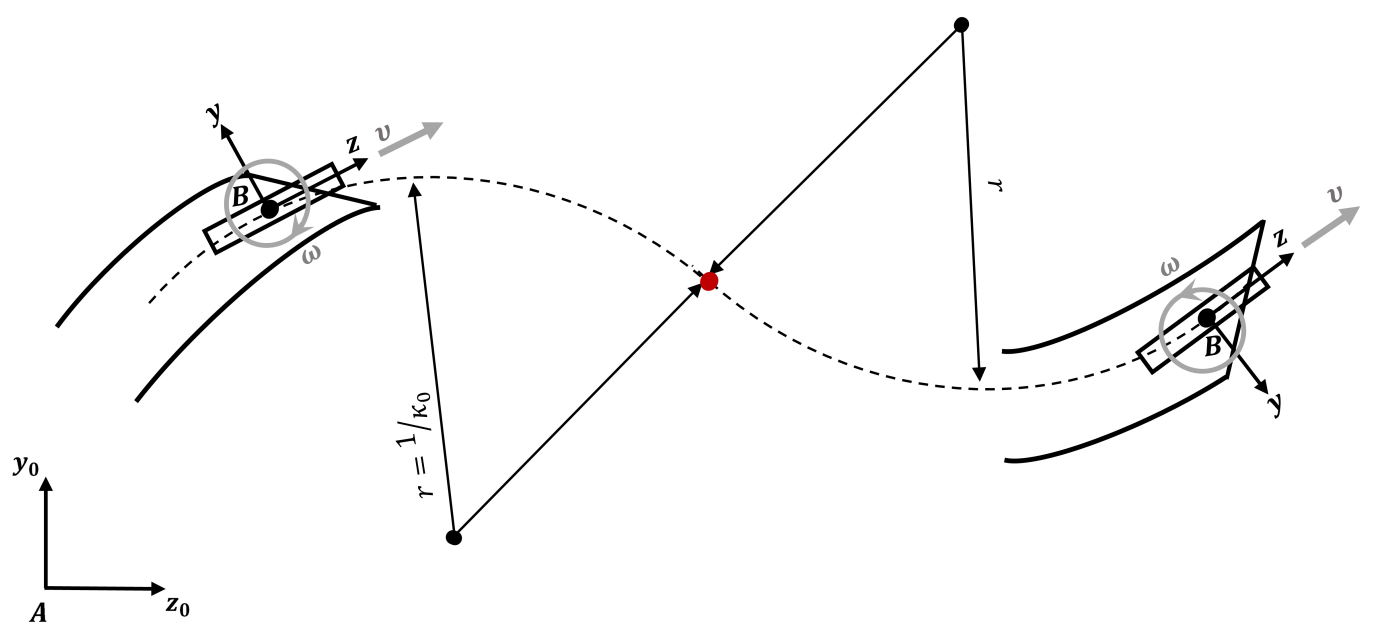

Figure 3.2: Planar unicycle model, adopted from [14].

$v$, the needle moves on a circular path with a constant radius of curvature $r=1 / \kappa_{0}$. Here we adapt the modified unicycle model for bevel-tip needle motion in which the angular velocity $\omega$ is proportional to the insertion velocity, i.e., $\omega=\kappa_{0} v$. While rotating the needle shaft by $180^{\circ}$ changes the bevel orientation and, consequently, changes the sign of angular velocity and curvature. As seen in Fig. 3.2, the $180^{\circ}$ change in bevel direction is applied at the center of the red bullet. Afterward, the 
needle tip continues to move along a circular path with the same radius of curvature but with a different rotational direction. It is clear that the two circular paths are tangent at the point of the intersection (red bullet).

As explained in [14], for planar motion, the needle tip configuration can be expressed by its pose $q=[y, z, \theta]^{T}$ where $y$ and $z$ are the coordinates of the origin of reference frame $B$ and $\theta$ is the orientation of $z$ axis of $B$, all expressed in inertial frame $A$. The deterministic motion of the bevel-tip needle is expressed as follows. $\lambda$ is either 1 or -1 depending on the bevel direction at $B$.

$$
\left[\begin{array}{c}
\dot{z} \\
\dot{y} \\
\dot{\theta}
\end{array}\right]=\left[\begin{array}{ccc}
\cos \theta & -\sin \theta & 0 \\
\sin \theta & \cos \theta & 0 \\
0 & 0 & \lambda
\end{array}\right] \times\left[\begin{array}{c}
v \\
0 \\
\kappa_{0} v
\end{array}\right]
$$

In the above kinematic model, by applying the insertion velocity in the $z$ direction at the needle base, needle tip follows a circular path in the $z$-y plane with an angular velocity of $\kappa_{0} v$ about an axis which is parallel to $x$-axis and located at $(y, z)=\left(-r_{0}, 0\right)$ expressed in the body frame $B$. The model parameter $\kappa_{0}$ depends on both some needle characteristics such as stiffness, diameter, bevel angle, and tissue properties such as stiffness and homogeneity. The dependency of $\kappa_{0}$ on the bevel angle and needletissue interaction has been studied in [32] and [76]. The authors in [14] obtained this parameter through different experiments on a tissue phantom. Chapter 5 discusses the importance of updating this parameter in a real-world application where the biological tissue has in-homogeneous properties. 


\subsubsection{Spatial Needle Motion}

In this section, we explain the 3D generalization of the standard nonholonomic 2D unicycle model as introduced in [14]. Two reference frames are shown in Fig. 3.3: $R F_{w}$, the fixed world reference frame and $R F_{n}$, the moving reference frame attached to the needle tip. In what follows, we have adapted a coordinate-free representation to exclude possible singularities in the system's equations. The orientation and position

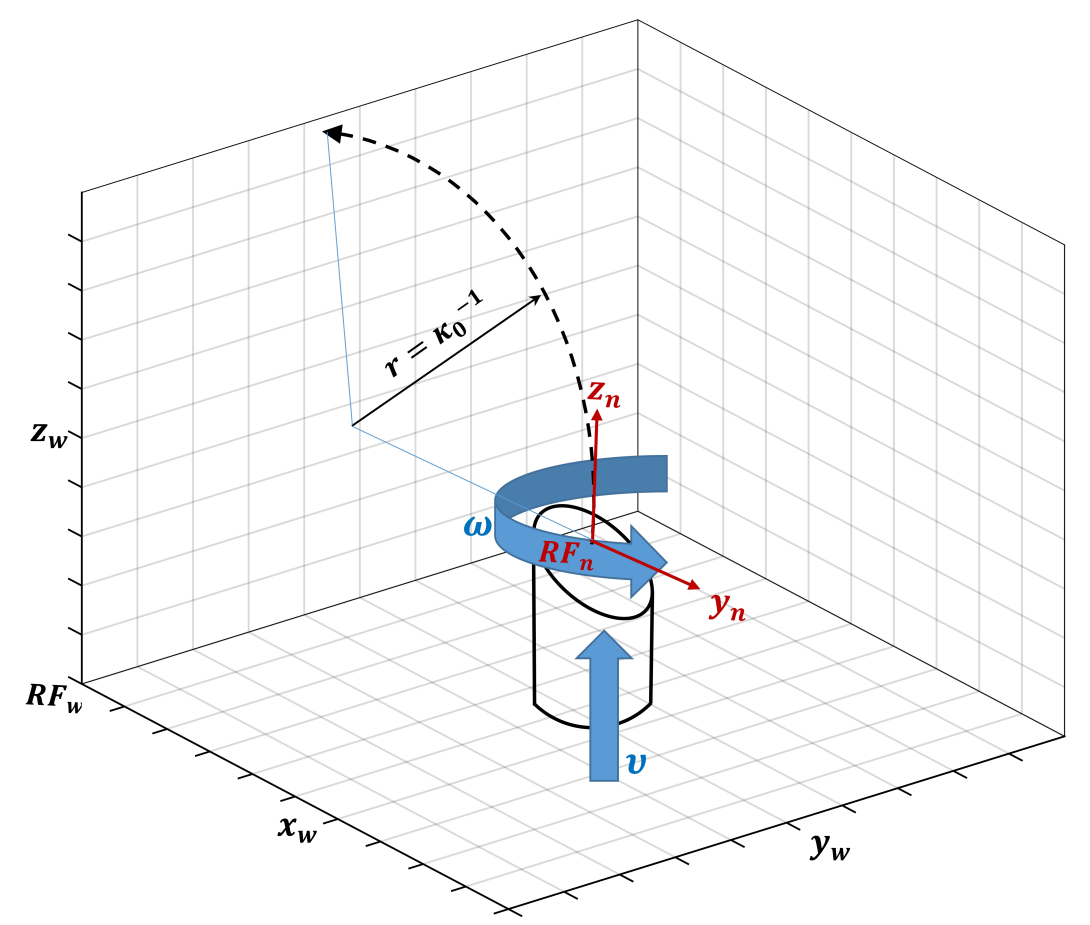

Figure 3.3: Spatial unicycle model.

of $R F_{n}$ with respect to $R F_{w}$ is denoted by $R_{w n} \in S O(3)$ and $p_{w n} \in \Re^{3}$, respectively. The associative rigid transformation is denoted through the homogeneous matrix $g_{w n} \in S E(3)$ as follows.

$$
g_{w n}=\left[\begin{array}{cc}
R_{w n} & p_{w n} \\
0^{T} & 1
\end{array}\right]
$$


Also, consider the following isomorphism

$$
\wedge:\left[\begin{array}{l}
v_{1} \\
v_{2} \\
v_{3}
\end{array}\right] \rightarrow\left[\begin{array}{ccc}
0 & -v_{3} & v_{2} \\
v_{3} & 0 & -v_{1} \\
-v_{2} & v_{1} & 0
\end{array}\right] \in s o(3), \vee:\left[\begin{array}{ccc}
0 & -v_{3} & v_{2} \\
v_{3} & 0 & -v_{1} \\
-v_{2} & v_{1} & 0
\end{array}\right] \rightarrow\left[\begin{array}{l}
v_{1} \\
v_{2} \\
v_{3}
\end{array}\right]
$$

where $s o(3)$ is the Lie algebra of $S O(3)$. The extension of the definition of the above mappings is according to the following.

$$
\wedge:\left[\begin{array}{l}
\boldsymbol{v} \\
\boldsymbol{\omega}
\end{array}\right] \rightarrow\left[\begin{array}{cc}
\hat{\boldsymbol{\omega}} & \boldsymbol{v} \\
0^{T} & 0
\end{array}\right] \in \operatorname{se}(3), \vee:\left[\begin{array}{cc}
\hat{\boldsymbol{\omega}} & \boldsymbol{v} \\
0^{T} & 0
\end{array}\right] \rightarrow\left[\begin{array}{l}
\boldsymbol{v} \\
\boldsymbol{\omega}
\end{array}\right]
$$

where $s e(3)$ is the Lie algebra of $S E(3)$, and $(\boldsymbol{v}, \boldsymbol{\omega}) \in R^{6}$. Based on the above definitions, $\boldsymbol{v}_{w n}^{b}$, the body-frame velocity of the needle tip $R F_{n}$ with respect to $R F_{w}$ is according to the following equation where the superscript b stands for body-frame.

$$
V_{w n}^{b}=\left[\begin{array}{c}
\boldsymbol{v}_{w n}^{b} \\
\boldsymbol{\omega}_{w n}^{b}
\end{array}\right]=\left(g_{n w}^{-1} \dot{g}_{n w}\right)^{\vee} \quad, \text { where } \quad \begin{gathered}
\boldsymbol{v}_{w n}^{b}=R_{w n}^{T} \dot{p}_{w n} \\
\boldsymbol{\omega}_{w n}^{b}=\left(R_{w n}^{T} \dot{R}_{w n}\right)^{\vee}
\end{gathered}
$$

\subsubsection{Nonholonomic Constraints and Control Inputs}

In this subsection, we extract the nonholonomic constraints of needle motion and introduce the kinematic model according to [14]. As shown in Fig. 3.3, it is observed the velocity of the origin of the frame $R F_{n}$ (needle tip) does not have any projections along the $x$ and $y$ axes of frame $R F_{n}$. This yields the following kinematic constraints 
where the unit vectors $e_{1}, e_{2}$, and $e_{3} \in \Re^{3}$ represent the standard basis.

$$
e_{1}^{T} \boldsymbol{v}_{w n}^{b}=e_{2}^{T} \boldsymbol{v}_{w n}^{b}=0
$$

As the needle is inserted inside the soft tissue, it moves along a curved trajectory with a radius of curvature $1 / \kappa_{0}$. The resultant angular velocity of the needle tip circular motion is about an axis parallel to the $x$-axis of frame $R F_{n}$ as follows.

$$
\frac{1}{\kappa_{0}} e_{1}^{T} \boldsymbol{\omega}_{w n}^{b}=e_{3}^{T} \boldsymbol{v}_{w n}^{b}
$$

Another source of needle motion is due to needle rotation about its shaft which results in a spin-like motion about the needle shaft. In fact, the needle tip does not have any angular motion about the $y$-axis of $R F_{n}$. This can be written as the following velocity constraint.

$$
e_{2}^{T} \boldsymbol{\omega}_{w n}^{b}=0
$$

The above four Pfaffian constraints can be rewritten in the matrix form as follows.

$$
\left[\begin{array}{cccccc}
1 & 0 & 0 & 0 & 0 & 0 \\
0 & 1 & 0 & 0 & 0 & 0 \\
0 & 0 & 1 & -1 / \kappa_{0} & 0 & 0 \\
0 & 0 & 0 & 0 & 1 & 0
\end{array}\right] V_{w n}^{b}=0 .
$$

It is simply can be investigated that the matrix in 3.9 is of rank 4 . Therefore, the right nullspace of the matrix in 3.9 is of rank 2. Consequently, we consider the basis 
$V_{1}$ and $V_{2}$ for the right nullspace of the matrix in 3.9 as follows.

$$
V_{1}=\left[\begin{array}{c}
\boldsymbol{v}_{1} \\
\boldsymbol{\omega}_{1}
\end{array}\right]=\left[\begin{array}{c}
e_{3} \\
\kappa_{0} e_{1}
\end{array}\right], \quad V_{2}=\left[\begin{array}{c}
\boldsymbol{v}_{2} \\
\boldsymbol{\omega}_{2}
\end{array}\right]=\left[\begin{array}{c}
0_{3 \times 1} \\
e_{3}
\end{array}\right]
$$

As seen in 3.10, vector $V_{1}$ corresponds to pure needle insertion, while vector $V_{2}$ corresponds to pure needle rotation about its shaft. In total, the needle tip velocity is the combination of the two permissible directions $V_{1}$ and $V_{2}$, which are defined in 3.10. As explained in section 3.2, it is assumed that the needle shaft motion is perfectly defined by the needle tip motion. In other words, the needle shaft solely replicates the control inputs applied at the needle base to the needle tip. We define the control input as $u=\left[u_{1}, u_{2}\right]^{T}$, where $u_{1} \triangleq v$ is the insertion speed and $u_{2} \triangleq \omega$ is the rotation speed about the needle shaft. As a result, the body-frame velocity of the needle tip has the form of

$$
V_{w n}^{b}=\left[\begin{array}{c}
0 \\
0 \\
u_{1} \\
\kappa_{0} u_{1} \\
0 \\
v \\
\kappa_{0} v \\
0 \\
u_{2}
\end{array}\right]=\left[\begin{array}{c}
0 \\
\omega
\end{array}\right] \Rightarrow\left\{\begin{array}{l}
\boldsymbol{v}_{w n}^{b}=\left[\begin{array}{l}
0 \\
0 \\
v
\end{array}\right] \\
\boldsymbol{\omega}_{w n}^{b}=\left[\begin{array}{c}
\kappa_{0} v \\
0 \\
\omega
\end{array}\right]
\end{array}\right.
$$

Therefore, the needle's one-parameter kinematic model can be expressed as follows 
where $n(t)$ is the needle tip position vector.

$$
\left\{\begin{aligned}
\dot{g}_{w n}(t) & =g_{w n}(t) \widehat{V}_{w n}^{b}(t), \quad \text { where } \widehat{V}_{w n}^{b}(t)=u_{1} \widehat{V}_{1}+u_{2} \widehat{V}_{2} \\
n(t) & =p_{w n}(t)
\end{aligned}\right.
$$

While $V_{w n}(t) \in s e(3)$ expresses the instantaneous linear and angular velocities of the needle tip in the world frame, the body-frame representation of $\widehat{V}_{w n}^{b}(t)$ has the following matrix form.

$$
\widehat{V}_{w n}^{b}(t)=\left[\begin{array}{cccc}
0 & -\omega(t) & 0 & 0 \\
\omega(t) & 0 & -v(t) / r_{0} & 0 \\
0 & v(t) / r_{0} & 0 & v(t) \\
0 & 0 & 0 & 0
\end{array}\right]
$$

When the body-frame needle tip velocity $\widehat{V}_{w n}^{b}(t)$ is constant, the equation in 3.12 turns to a linear ordinary differential equation as follows which has a relatively simple analytic solution.

$$
g_{w n}(t)=g_{w n}(0) \exp \left(t \widehat{V}_{w n}^{b}\right)
$$

Since the distribution $\Delta=\operatorname{span}\left\{V_{1}, V_{2}\right\}$ is not involutive, the system explained in (3.12) is nonholonomic with a degree of nonholonomy equal to 4 , which can be investigated by successive Lie brackets. Therefore, the system is controllable. In Chapters 4 and 5, we explore the path-planning problem for the system. 


\subsection{Variable-Curvature Needle Motion}

In this section, we explain the reason for adapting the variable-curvature model of needle motion. Also, we explicitly describe the method which generates the variablecurvature trajectories of the needle tip motion.

\subsubsection{Reachability Region}

It is experimentally observed in [14] that when a flexible bevel-tip needle is inserted inside the soft tissue and under the assumptions explained in 3.2, the needle tip follows a path of an approximately constant curvature $\kappa_{0}$, called the needle natural curvature or needle maximum curvature. It means when the needle motion is driven by pure insertion at the needle base, the path of the needle tip attains the maximum curvature. However, by incorporating rotation into insertion, other curvatures $\kappa$ rather than $\kappa_{0}$ can be achieved. Assume that for a given needle tip initial configuration, the needle tip is supposed to reach the desired position in a $3 \mathrm{D}$ space. This can be done through a correct choice of control input sequence applied at the needle base. In the case of constant-curvature assumption, it is intuitively clear that the task of reaching the desired position is more complicated through a more complex set of control inputs including insertion, retraction to a certain distance, reorientation of the bevel direction, and reinsertion. On the other hand, when the constant-curvature constraint is relaxed, due to more freedom in the needle motion, the task of reaching the desired position seems to be more feasible compared to the constant-curvature assumption. This is presented schematically in Fig. 3.4. As seen, when the needle is purely inserted at the base, the needle tip follows a path of a constant radius of curvature $r_{0}=1 / \kappa_{0}$ at the direction of the bevel. Rotating the needle shaft at its base changes the direction of the bevel and, accordingly, changes the plane containing the needle circular motion. Therefore, for a given initial configuration the needle tip 


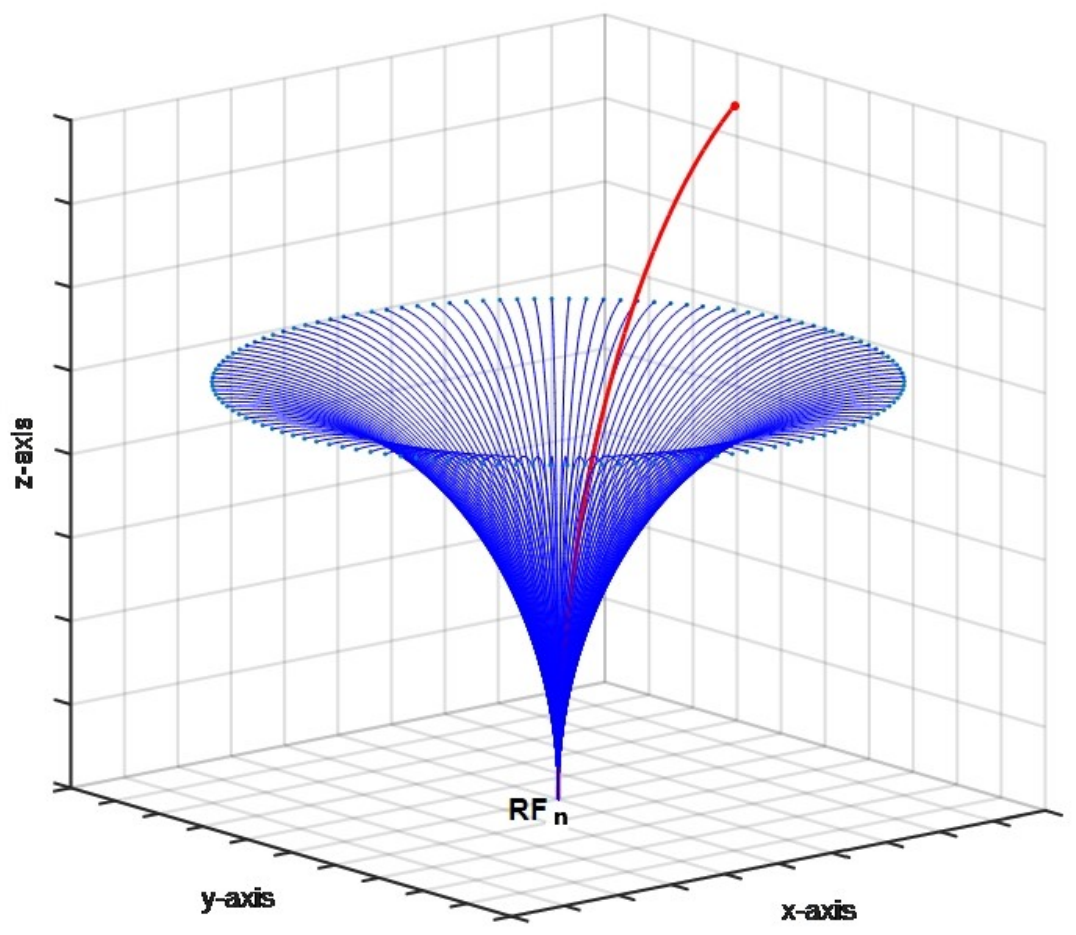

Figure 3.4: Different possibilities of the needle tip circular motion in the local coordinate frame $R F_{n}$ are shown in blue arcs of curvature $\kappa_{0}$. For the constantcurvature case, the reachability region belongs to the surface of the volume (blue arcs); however, using the DC approach, the extended reachability region includes the space inside the volume, too. A red curve is a representative path in the extended reachability region, adopted from [15].

only has access to the region located on the surface of the volume shown in Fig. 3.4. This is called the needle tip reachability region which is a very limited subset of the configuration space. Thus, in order to expand the needle tip reachability region which in turn eases the path-planning process significantly, it is necessary to generate needle tip trajectories of variable curvatures. We will experimentally verify the importance of the variable-curvature needle tip motion in Chapters 4 and 5 . 


\subsubsection{Duty-Cycle Approach}

The model introduced in 3.12 represents the constant-curvature needle motion inside soft tissue for the needle that is flexurally flexible and torsionally stiff. The constant-curvature constraint can severely reduce the needle tip path possibility of reaching a target in the configuration space. This constraint is first relaxed by Engh et al. [77] and later by Minhas et al. [16] through the duty-cycled spinning of the needle base. Engh et al. in [77] presented a novel active approach to creating variablecurvature trajectories for bevel-tip needles. It is shown in [14] that through pure needle insertion at the needle base, the flexible bevel-tip needle bends on a circular path of curvature $\kappa_{0}$, where the amount of needle maximum curvature $\kappa_{0}$ depends on the relative stiffness of the needle and the tissue as well as the bevel angle [76]. Through the needle shaft rotation at the needle base, the plane of circular motion varies respectively. This type of steering the bevel-tip needle controls the direction of the needle curvature but is not capable of changing the amount of the needle path curvature. On the other hand, by spinning the needle (simultaneously rotating the needle shaft at a rate greater than the insertion speed), straight trajectories can be achieved. Given these two facts, variable-curvature trajectories can be achieved by combining short segments of straight trajectories $T_{\text {spin }}$, followed by short segments of curved trajectories $T_{i n s}$. It should be noted that in order to keep the needle tip on the same plane of interest during spinning intervals, the needle tip has to maintain the same angle of rotation at the end of each spinning interval. By correctly adjusting these segments, any desired curvature $0 \leq \kappa \leq \kappa_{0}$ between the two limiting values, i.e., 0 and $\kappa_{0}$, with and without spinning, can be obtained, and, consequently, a proportional control of the desired needle curvature $\kappa$ can be achieved as: $\kappa=$ $\kappa_{0}(1-\alpha)$ where $\alpha=T_{\text {spin }} /\left(T_{\text {spin }}+T_{\text {ins }}\right)$ is the duty-cycle factor and $0 \leq \kappa \leq \kappa_{0}$. This method is called the Duty-Cycle spinning of the bevel-tip needle (DC approach). 
It is clear that longer non-spinning intervals lead to paths with a curvature closer to $\kappa_{0}$, while longer spinning intervals lead to paths closer to a straight line. As a result, by correctly choosing the spinning interval out of the duty-cycle period, not only can the proportional control of the needle tip direction be achieved as before in [14], but also the proportional control of the amount of the needle tip curvature can be attained. This observation is qualitatively addressed in [77], while later Minhas et al. in [16] developed the kinematic model for needle steering using the DC approach. They quantitatively obtained the needle tip trajectory using the needle natural curvature and the duty-cycled spinning of the needle. We follow their notation in the rest of this subsection to express the kinematics of needle steering based on the DC approach.

Let the duty cycle of spinning as $D=\tau / T$, where $\tau$ is the time duration of the spinning interval while $T$ is the duty cycle period, i.e., the summation of time duration spent on both the spinning and non-spinning intervals. In the new kinematic model, the control input related to the insertion speed is similar to that of $3.12, u_{1}=v$, however the control input related to the needle shaft angular motion $u_{2}$ is modified as follows, while ensuring that $\widehat{\omega} \gg v$.

$$
u_{2}(t)=\omega(t)= \begin{cases}\widehat{\omega}, & j T \leq t \leq T(j+D), \quad j=0,1,2, \ldots \\ 0, & \text { otherwise }\end{cases}
$$

The modified kinematic model for duty-cycled needle motion has the following form.

$$
\left\{\begin{aligned}
g_{w n}(t) & = \begin{cases}g_{w n}(0) \exp \left(v \widehat{V}_{1}+\widehat{\omega} \widehat{V}_{2}\right) t, & j T \leq t \leq T(j+D), j=0,1,2, \ldots \\
g_{w n}(0) \exp \left(v \widehat{V}_{1}\right) t, & \text { otherwise }\end{cases} \\
n(t) & =p_{w n}(t)
\end{aligned}\right.
$$


Using the DC approach, the reachability region of the needle tip for a given initial configuration varies. Regarding Fig. 3.4, the new reachability region includes both the surface of the volume as well as the space inside the volume. A representative curve in red is added to the figure, which belongs to the extended reachability region of the needle tip. This substantial increase in the reachability region eases the task of reaching a desired position in the 3D space, using a sequence of control actions, which in turn facilitates the path-planning task of the bevel-tip needle motion. Fig. 3.5 shows the experimental results obtained in [16] for different trajectories of a flexible bevel-tip needle at various values of the duty cycle. Besides, Fig. 3.6 shows the simulated results for bevel-tip needle trajectories obtained at different values of the duty cycle. Both figures confirm the ability of the DC method in generating variable curvatures up to the needle maximum curvature. As mentioned later by Patil et al. in [18], the relationship between $\kappa$ and $\alpha$ is not always linear. In general, it has the form of $\alpha=h[\kappa]$ where $\mathrm{h}$ is a function of needle-tissue mechanical properties. From an experimental point of view, since the DC approach is associated with the continuous rotation of the bevel-tip needle, using some instrumentation such as forcetorque sensors and electromagnetic trackers is prevented due to the cable wind-up problem. The authors in [78] presented two control methods to overcome hardware limitations such as cable wind-up in the duty-cycled spinning of steerable needles.

\subsection{Summary}

This chapter provides a mathematical representation of the bevel-tip needle motion during insertion inside soft tissue. The necessary assumptions made are discussed as well. As mentioned earlier, the needle tip follows a path of constant curvature during insertion inside soft tissue. Subsequently, both the planar and spatial unicycle models, including the nonholonomic constraints and control inputs, are elaborated 

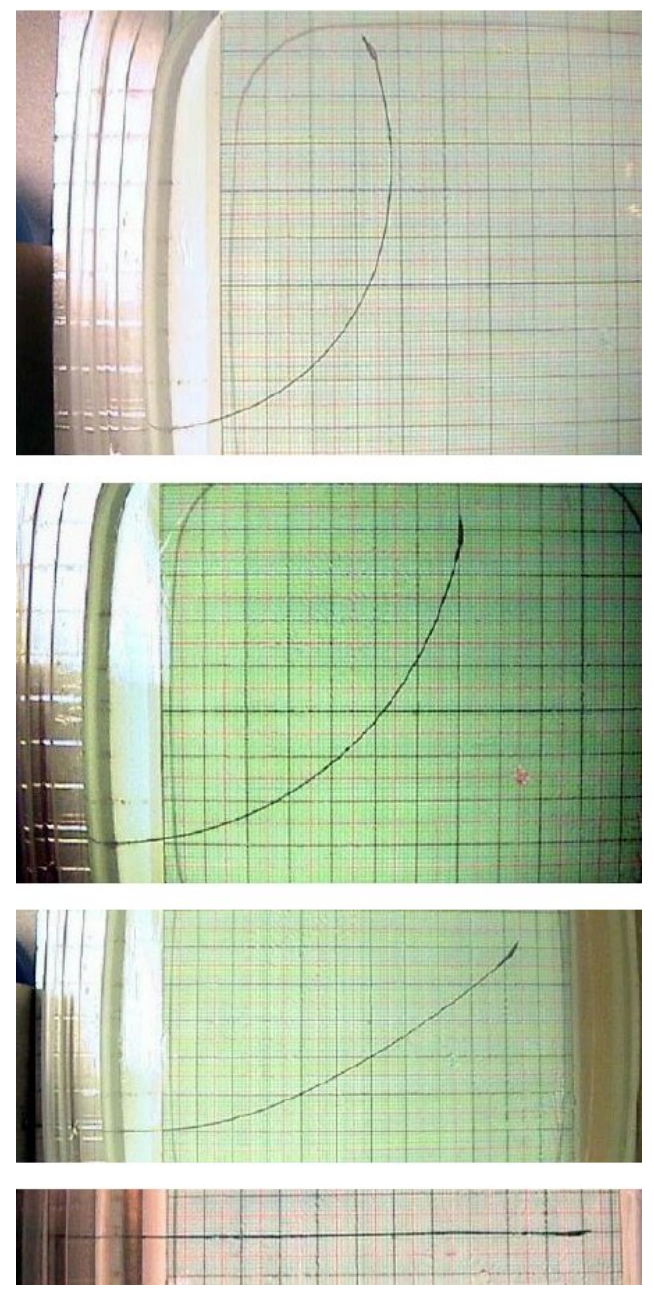

Figure 3.5: Experimentally obtained trajectories of a flexible bevel-tip needle at various duty cycles. (a) 0 duty cycle (no spinning). (b) 33 duty cycle. (c) 67 duty cycle. (d) 100 duty cycle (constant spinning), reprinted from [16] (c) 2007 IEEE).

on. The concept of the reachability region, which plays an instrumental role in pathplanning algorithms, is explained, as well. More importantly, the active method of the duty-cycled spinning of the needle in generating variable-curvature needle paths is introduced. 


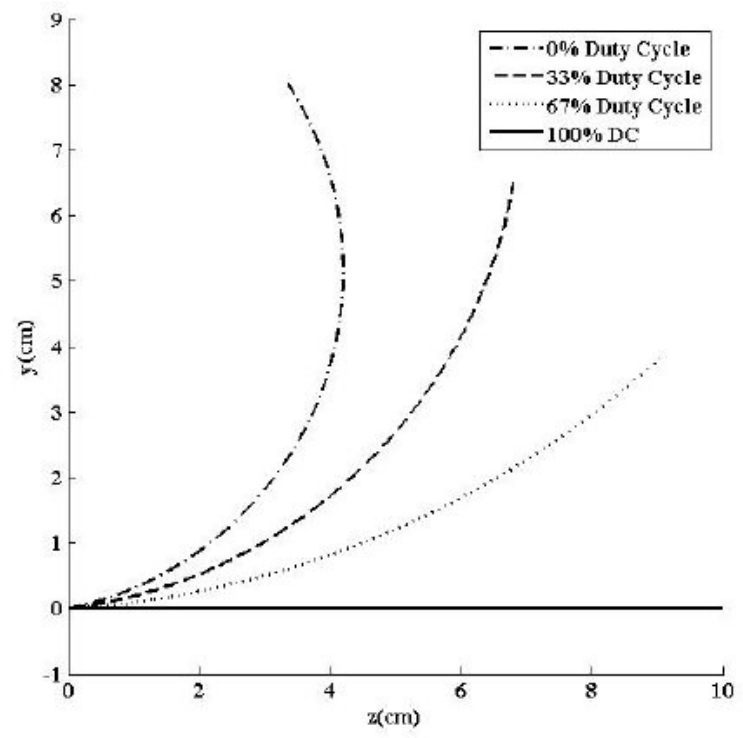

Figure 3.6: Simulated trajectories of a flexible bevel-tip needle at various duty cycles, reprinted from [16] (C) 2007 IEEE). 


\section{Chapter 4}

\section{D Preoperative RRT Path Planner for Steerable Bevel-Tip Needles in Multiple-Targets Soft Tissue}

\subsection{Introduction}

This chapter discusses the preoperative planning for the planar motion of a steerable bevel-tip needle. Chapter 3 presented both planar and spatial motion kinematics of the flexible bevel-tip needle inside soft tissue. The needle-tissue interaction model is characterized by a single parameter, $\kappa_{0}$, which is the needle's natural curvature and depends on the mutual characteristics of the needle and soft tissue. However, in real-world applications, the one-parameter model does not reflect the inherent complications of the needle-tissue interaction during insertion in biological soft tissue. Tissue deformation, needle deflection, tissue inhomogeneity, patient variability, and associated uncertainty are some of the major complications. Therefore, for real-world applications, closed-loop control and intraoperative planning is required, which is the focus of Chapter 5. Yet, preoperative planning has received a great deal of scholarly attention. Most of the proposed preoperative planning methods are experimentally 
validated using tissue phantoms, which have more consistent properties and provide a more controlled environment to study and analyze the system performance.

The rest of the chapter is organized as follows. First, Section 4.2 introduces the preoperative planar single-target RRT-based planning algorithm, which starts with a discussion of Rapidly Exploring Random Trees as a useful tool in path planning of bevel-tip needle motion in Subsection 4.2.1. The multiple-target version of our planner is developed in Section 4.3. Section 4.4 highlights the significance of the DC approach through simulation results. Finally, Section 4.5 concludes the chapter.

\subsection{Single-Target RRT-Based Path-Planning Al- gorithm}

One issue featuring prominently in robotic needle insertion systems is steering and path planning of the steerable needles inside soft tissue [23]. Currently, needle insertion procedures are based on using straight rigid needles in operating rooms to reach targets. However, in more general cases where targets are not accessible through straight-line trajectories, a steerable needle is required to follow a more complex trajectory in order not to collide with impenetrable objects and damage any sensitive tissue structures. Hence, the concept of trajectory planning for steerable needles plays a crucial role in needle steering systems. Compared to symmetric-tip needles, higher maneuverability can be achieved through tip steering of steerable bevel-tip needles with an asymmetric tip shape, particularly in deeper insertion depths, and the task of path planning proves more feasible [14], [79]. This section introduces our planner for a single target, which is an improved variant to the $2 \mathrm{D}$ version of [18]. 


\subsubsection{Rapidly Exploring Random Trees}

One major categorization of path-planning approaches is based on the trade-off between optimality and speed. In the former case, a cost function is usually defined to numerically represent the path-planning problem. It could be any combinations of different objectives such as minimum control effort, minimum needle inserted length, maximum clearance with obstacles, and minimum targeting offset. However, one serious limiting factor associated with optimization-based planning approaches is their time-consuming feature which increases with an increase in problem dimensionality. Consequently, this restricts their applications to open-loop planning.

On the other hand, in recent years, sampling-based approaches have become more popular in path-planning problems. These algorithms are advantageous in that they provide fast solutions to difficult problems; however, the solutions they provide are not optimal. They do not provide guarantee in finding a solution if one exists. This property is called the completeness of an algorithm. On the other hand, these algorithms provide a weaker version of completeness, called probabilistic completeness. It means that given sufficient time (which could be infinite time), the algorithm provides a solution if any exists. Two most commonly used sampling-based algorithms are Probabilistic Roadmap Method (PRM) [26], [28] and Rapidly-exploring Random Trees (RRT) [27]. Both RRT and PRM are based on random sampling of the free configuration space but with different specifications. PRM mainly focuses on optimality by randomly sampling the free configuration space. Then using a local planner, it checks for collision-free connections of the already sampled states. Once the roadmap has been constructed in an off-line fashion, the globally optimal path can be computed for the given grid, using a fast graph-based search algorithm. Moreover, PRM algorithms are complete. However, both optimality and completeness are guaranteed up to the given grid resolution. It is known that there is a trade-off between the size 
of the grid and computation time. In other words, a high-resolution grid, where the solution is more convergent to the true optimal solution, has higher time complexity.

In contrast to PRM, the RRT method concentrates on a fast exploration of the configuration space without providing any guarantee of optimality. Instead of discretizing the configuration space and, consequently, using a graph-based search algorithm, in the RRT, each edge and vertex is created incrementally by sampling the free continuous configuration space. The RRT is biased toward exploring the unexplored configuration space by progressively growing a tree from the root node toward a target. For the recent obstacle-free randomly sampled state, the RRT planner checks for a feasible connection between the sampled state and the most updated version of the constructed tree. If one is found, then both the sampled state and the associated connection are added to the tree as its new vertex and edge. The solution of an RRT path-planning algorithm is either a failure or a feasible path. In other words, in limited time, it does not provide a guarantee of completeness. However, the algorithm is probabilistically complete. The RRT Path-Planning algorithms work well under kinematic differential constraints and especially for systems with high dimensional configuration spaces, consequently, making them a good candidate for steerable bevel-tip needle path-planning problems under geometric and nonholonomic constraints.

\subsubsection{Biasing the Algorithm}

For high-dimensional problems like the one for the needle steering system, different heuristics have been introduced in the literature for easing computational expenses. It is known that the distribution of the vertices in the tree depends on the sampling distribution [57]. As a result, instead of sampling the tree uniformly, biasing the sampling toward the goal is preferred to improve the planner's efficiency. In this regard, two types of biasing are introduced in [18], which have been used in our work, 
too. First, the growth of the tree is biased toward the goal by sampling from the goal region with a higher probability than the rest of the free configuration space. This way of biasing could be more effective, particularly when the target region is greater than a circle with a radius of a millimeter. Second, once each node has been added to the tree, the algorithm tries to connect the recently added node to a randomly sampled point in the target region. If the connection is found, then the algorithm terminates since it has reached the target region. Otherwise, the algorithm continues until either it reaches the target region or the maximum number of iterations is violated. In the former case, the algorithm returns a feasible path as its solution, while in the latter case, the algorithm returns failure for the prespecified maximum number of iterations.

In what follows, we first introduce our planner for a single target, which is similar to the $2 \mathrm{D}$ version of [18] but with two added sampling biases: one for $\theta_{0}$ and the other for $P_{\text {rand }}$. The planar bevel-tip needle motion kinematics is explained in detail in Subsection 3.3.1. The needle tip configuration is denoted by its pose in the plane through $q=[y, z, \theta]$. As shown in Fig. 4.1, the needle tip can be explicitly characterized by its tip position $P=(y, z)$ and tip orientation $\theta$, while parameter $\lambda$ represents the binary status of the bevel direction. For a desired needle tip configuration $q_{A}=\left[y_{A}, z_{A}, \theta_{A}\right]^{T}$ and any arbitrary sampled position $p_{B}=\left(y_{B}, z_{B}\right)$, there exists a unique circular arc of radius $r_{i}$ connecting $q_{A}$ to $p_{B}$ in the plane containing $q_{A}$ and $p_{B}$. However, due to the mentioned constraint on the needle's circular motion, it is not always feasible for any arbitrary sampled point $p_{B}$ to be reached by the needle tip at configuration $q_{A}$ through a single curve. In other words, the needle tip at $q_{A}$ only has access to a certain portion of the configuration space, which is called the reachability region. Mathematically, it is defined by $r_{i} \geq 1 / \kappa_{0}$.

Like [15], [18], [12], we use variable curvature trajectories to navigate the needle toward the target. As mentioned in [52], steerable needle kinematics equations are invariant with respect to time scaling. It means that the path traced by the needle 

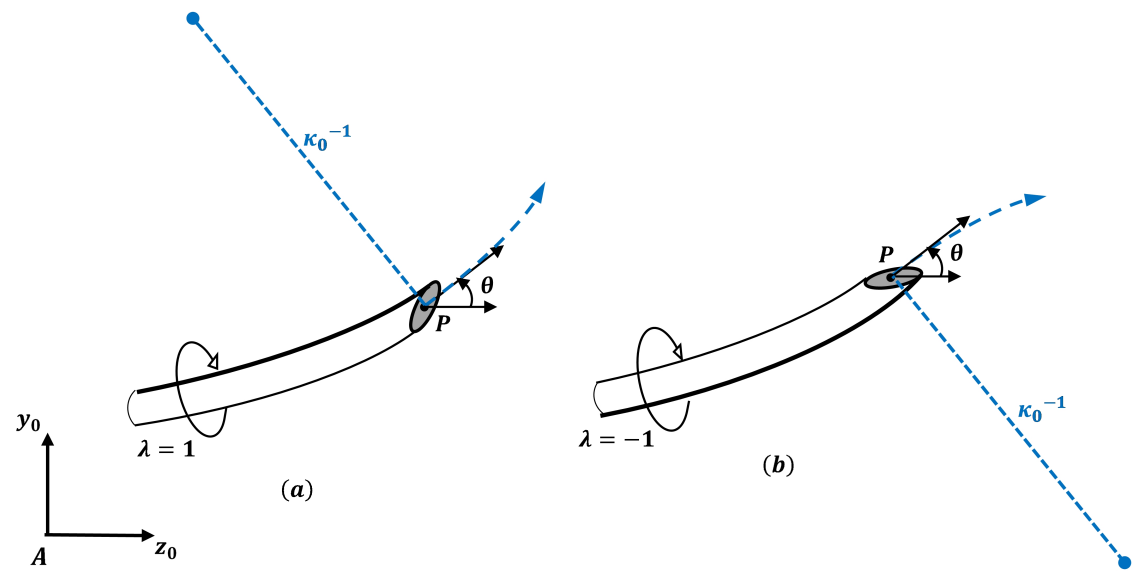

Figure 4.1: Bevel-tip needle configuration in a plane. Parameters $P$ and $\theta$ represent the needle tip position and orientation, respectively. a) Relates to the bevel left direction, $\lambda=1$, where the needle tip follows a path of constant curvature $\kappa_{0}$ counterclockwise. b) By flipping the bevel direction to the right, $\lambda=-1$, the needle tip position does not change; however, by further inserting the needle, it follows the circular path with the same curvature but clockwise, adopted from [17].

does not change if the control inputs $v(t)$ and $\omega(t)$ are scales by the same factor. As a result, for path planning purposes, one can assume without loss of generality that $v(t)=v_{\text {fixed }} \equiv 1$ and, then, parameterize the needle trajectory with respect to the needle insertion length instead of time. This geometric representation of the needle path facilitates planning calculations. Consequently, the needle path to the target will be the concatenation of circular arc segments with different curvatures. As explained in [80], a typical piece-wise circular arc segment $C_{i}$, characterized by $\left(l_{i}, r_{i}, \phi_{i}\right)$ is shown in Fig. 4.2. We define the needle tip pose at $B$ relative to $A$ by $\left[y_{i}, z_{i}, \theta_{i}\right]^{T}$, where $\theta_{i}=\theta_{A}-\theta_{B}$ and $\left(y_{i}, z_{i}\right)$ are the relative coordinates of $B$ expressed in $A$. Therefore, given the needle tip pose at $A$ and randomly sampled point's position at $B$, the parameters of the respective circular arc segment $C_{i}$ can be uniquely obtained 


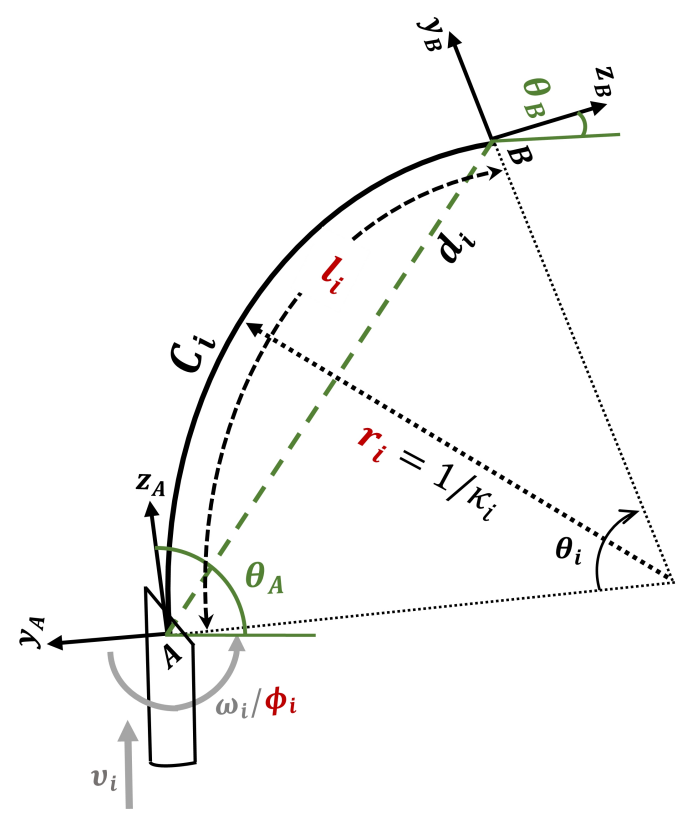

Figure 4.2: Geometric representation of a piece-wise circular arc segment $C_{i}$ which is characterized by $\left(l_{i}, r_{i}, \phi_{i}\right)$. Given the needle tip configuration at $\mathrm{A}\left(X_{A}=\right.$ $\left.\left[y_{A}, z_{A}, \theta_{A}\right]^{T}\right)$ and sampled point's position at $\mathrm{B}\left(P_{B}=\left[y_{B}, z_{B}\right]^{T}\right)$, arc parameters can be uniquely obtained and used in the planner, adopted from [18].

according to (4.1)-(4.2).

$$
\begin{aligned}
& r_{i}=\frac{y_{i}^{2}+z_{i}^{2}}{2\left|y_{i}\right|} \\
& l_{i}=r_{i} \theta_{i}=r_{i} \operatorname{atan} 2\left(z_{i}, r_{i}-\left|y_{i}\right|\right)
\end{aligned}
$$

The parameter $\phi_{i}$ represents the change in the bevel tip orientation and is equivalent to flipping the bevel to left or right. To make the transition of the needle tip from $A$ to $B$ possible using control inputs $u_{i}=\left[v_{i}, \omega_{i}\right]^{T}$ at the needle base, first, the needle bevel tip is reoriented by $\phi_{i}{ }^{\circ}$ about the $z_{A}$-axis. For planar motion, this change of bevel orientation is either $0^{\circ}$ or $180^{\circ}$ depending on the sign of the curvature. If there is a change in the curvature sign, the bevel tip is flipped by $180^{\circ}$. Otherwise, it is 
kept unchanged $\left(\phi_{i}=0^{\circ}\right)$. Afterwards, the needle tip has to move along $C_{i}$ over time interval $\Delta_{i}$. Using the DC approach in [58], time interval $\Delta_{i}$ is split into a number of duty-cycling periods $T_{i}$, where $T_{i}=T_{\text {ins }_{i}}+T_{\text {spin }_{i}}$ is the summation of spinning and nonspinning intervals $T_{\text {spin }}$ and $T_{i n s_{i}}$, respectively. This is shown in Fig. 4.3. According to [58], during $T_{s p i n_{i}}$, the rotational velocity of the needle $\omega_{s p i n_{i}}$ has to be much greater than the insertion velocity $v_{i}\left(\omega_{s p i n_{i}} \gg v_{i}\right)$. For given $\omega_{s p i n_{i}}$, the spinning interval is $T_{\text {spin }}=2 k \pi / \omega_{\text {spin }}$. Therefore, $T_{i}=T_{\text {spin }} / \alpha_{i}$ and $T_{i n s_{i}}=T_{i}\left(1-\alpha_{i}\right)$. For given

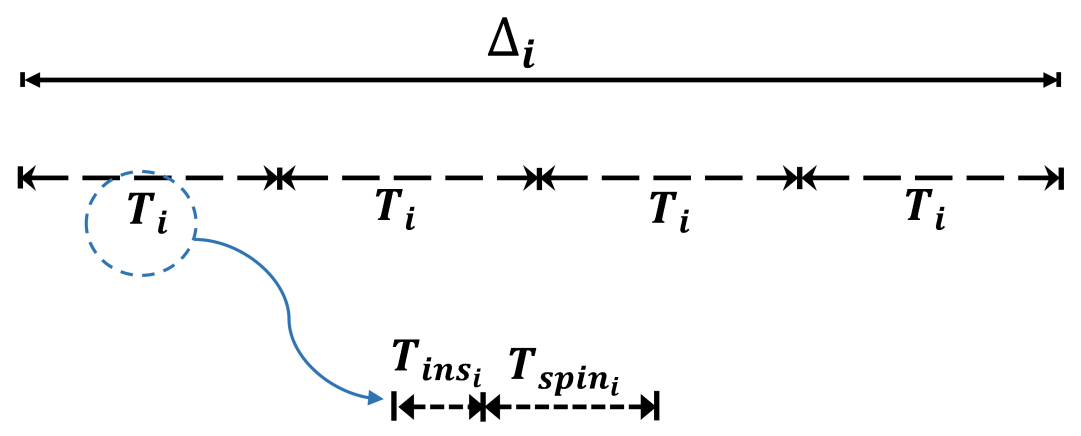

Figure 4.3: Time duration $\Delta_{i}$ is split into four duty-cycled intervals $T_{i}$. Each $T_{i}$ is composed of two intervals: spinning interval $T_{\text {spin }_{i}}$ and non-spinning interval $T_{\text {ins }}$, adopted from [18].

$\Delta_{i}$ and duty-cycle factor $\alpha_{i}$, the control input $v_{i}(t)=l_{i} / \Delta_{i}$ and $\omega_{i}(t)$ is according to (4.3), where $j \in\left\{0,1, \ldots, \Delta_{i} / T_{i}\right\}$.

$$
\omega_{i}(t)= \begin{cases}\omega_{\text {spin }_{i}}, & \text { if } j T_{i}<t \leq j T_{i}+T_{\text {spin }} \\ 0, & \text { if } j T_{i}+T_{\text {spin }_{i}}<t \leq(j+1) T_{i}\end{cases}
$$




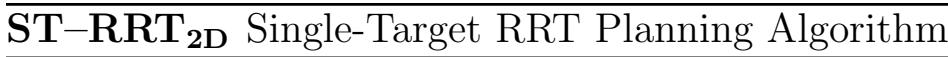

$$
\begin{aligned}
& \text { 1: } \mathfrak{T} \leftarrow \text { Initialize_path }\left(P_{0}, P_{\text {target }}, K\right) \\
& \text { 2: while }\left(\mathfrak{T} \cap P_{\text {target }}=\emptyset \& k \leq K\right) \\
& \text { 3: } \quad P_{\text {rand }} \leftarrow \text { obstacle-free_random_position }\left(\Re^{2}\right) \\
& \text { 4: } \quad Q_{\text {reach }} \leftarrow \text { reachable_set }\left(P_{\text {rand }}, \mathfrak{T}\right) \\
& \text { 5: } \quad X_{\text {near }} \leftarrow \text { closest_neighbor }\left(Q_{\text {reach }}, P_{\text {rand }}, \rho(.)\right) \\
& \text { 6: } \quad(l, r, \phi) \leftarrow \text { circular_arc }\left(X_{\text {near }}, P_{\text {rand }}\right) \\
& \text { 7: } \quad u \leftarrow \text { control_inputs }((l, r, \phi), \Delta) \\
& \text { 8: } \quad X_{\text {new }} \leftarrow \text { new_state }\left(X_{\text {near }}, u, \Delta\right) \\
& \text { 9: } \quad \text { if collision_free }\left(X_{\text {near }}, u, \Delta, X_{\text {new }}\right) \\
& \text { 10: } \quad \mathfrak{T} \leftarrow \text { add_vertex }\left(X_{\text {new }}\right) \\
& \text { 11: } \quad \mathfrak{T} \leftarrow \text { add_edge }\left(X_{\text {near }}, u, \Delta, X_{\text {new }}\right) \\
& \text { 12: } \quad \text { end } \\
& \text { 13: } \quad \text { if } P_{\text {new }} \in P_{\text {target }} \\
& \text { 14: } \quad \mathfrak{P} \leftarrow \text { extract plan }(\mathfrak{T}) \\
& \text { 15: end } \\
& \text { 16: } \quad k=k+1 \\
& \text { 17: end } \\
& \text { 18: return } \mathfrak{P}
\end{aligned}
$$

Therefore, by applying the control inputs $\omega_{i}$ and $v_{i}$ during the time interval $\Delta_{i}$, the needle tip follows a circular path with a curvature $\kappa_{i}=1 / r_{i}$. As mentioned earlier, by choosing the right sequence of control actions for the whole insertion time, a path connecting the needle entry point to the target region can be calculated as an integration of the different circular arc segments of variable curvatures $\kappa_{i}$. In the following, the RRT planning algorithm for a single target, which is outlined in $\mathrm{ST}-\mathrm{RRT}_{2 \mathrm{D}}$, is elaborated.

Inputs: the needle's entry position $P_{0}$, target region $P_{\text {target }}$ which is considered as a circle with a radius of $1 \mathrm{~mm}$ (the radius of the minimum-sized detectable object through ultrasound measurements [81]), and the maximum number of iterations $K$. 
Outputs: needle planned trajectory $\mathfrak{P}$, extracted from tree $\mathfrak{T}$, which connects $P_{0}$ to $P_{\text {target }}$.

First, given $P_{0}=\left[y_{0}, z_{0}\right]$, needle initial orientation $\theta_{0}$ is randomly sampled through a biased strategy. The critical effect of biasing the algorithm toward the goal to improve the planner's efficiency has been emphasized in [18]. Otherwise, the planner's efficiency degrades substantially to the point of rendering the algorithm unresponsive. Table 4.1 shows the results of the planner's performance in the absence of biasing. The parameters of cost, time, and $N_{n d s}$ refer to needle insertion length, computation time, and the number of nodes in the tree, respectively. Two different scenarios are chosen for a single target in the configuration shown in Fig. 4.4, namely $T_{i o}=(1,8.5)$ and $T_{b o}=(4,8.5)$, which refer to the cases where the target is in front of and behind the obstacle, respectively. This is to verify the performance of the planner with respect to the target location. Also, in order to see the sensitivity of the planner to the target region, we have also provided the results for the case, where the radius of the target circle has doubled, i.e. $R=0.2 \mathrm{~cm}$. The results correspond to 100 simulations, in each of which the maximum number of iteration $K$ and needle natural curvature $\kappa_{0}$ are set to 10000 and $0.2 \mathrm{~cm}^{-1}$, respectively. The following implications can be deduced from Table 4.1.

1. Since the results relate to running the algorithm one time, the large numbers for both the average time and the average number of nodes, $N_{n d s}$, in the tree point to the necessity of biasing the algorithm.

2. The standard deviations for the cost values are low, while the standard deviations for the time and $N_{n d s}$ are very large. Although the algorithm is based on uniform random sampling of the configuration space, regarding the cost values, there are limited options for the planner to choose the path toward the target for a given needle initial location; this is due to needle poor maneuverability in 
the limited size of the workspace. As either the workspace dimensions or the needle natural curvature increases, so does the standard deviation of the cost.

3. For the smaller target region $(r=0.1 \mathrm{~cm})$, the time values are considerably large. This is because as $N_{n d s}$ increases, the planner's efficiency may drop accordingly due to the increased volume of computations (this is especially true when the tree expands uniformly in the configuration space).

4. A comparison of the computation time for the larger target region with that for the smaller one points to a significant improvement for the larger target region. Although the average $N_{n d s}$ in the tree for the larger target region is about 2 or 3 times less than that for the smaller one, item 3 , as stated above, explains the great improvement in computation time.

Table 4.1: Numerical analysis of the performance of the RRT-based planner for a single target of different locations and target regions under no algorithm biasing condition. The results correspond to $n=100$ simulations.

\begin{tabular}{|c|c|c|c|c|c|c|c|}
\hline \multicolumn{2}{|c|}{$n=100$} & \multicolumn{2}{c|}{ cost $(\mathrm{cm})$} & \multicolumn{2}{c|}{ time $(\mathrm{s})$} & \multicolumn{2}{c|}{$N_{\text {nds }}$} \\
\hline target radius & target choice & mean & StDev & mean & StDev & mean & StDev \\
\hline \multirow{3}{*}{$r=1 \mathrm{~mm}$} & $T_{i o}$ & 8.68 & 0.105 & 21.82 & 80.64 & 1667.66 & 1390.28 \\
\cline { 2 - 8 } & $T_{b o}$ & 9.87 & 0.331 & 32.41 & 108.58 & 1702.84 & 1388.71 \\
\hline \multirow{3}{*}{$r=2 \mathrm{~mm}$} & $T_{i o}$ & 8.71 & 0.15 & 0.88 & 0.40 & 498.74 & 511.85 \\
\cline { 2 - 8 } & $T_{b o}$ & 9.99 & 0.39 & 0.99 & 0.49 & 674.14 & 676.46 \\
\hline
\end{tabular}

Apart from biasing the algorithm toward the target as explained in [18], here we limit the height of the sampled point to be around the target. In addition, we apply a normal distribution to sample $\theta_{0}$ and set the mean value to the tangent line angle between the start and target locations. It should also be stressed that the effect of parameter $\theta_{0}$ on the planning performance is critical. In most of the works introduced 
in the literature [58], [15], [64], and [18], parameter $\theta_{0}$ is considered as a given to the problem and is obtained through preoperative medical images. However, in a realworld scenario, where the target and obstacles may move and the tissue may deform, the desired value of $\theta_{0}$ cannot be necessarily taken for granted. In particular, for the planar motion, when the needle entry region is very limited due to the anatomy of the body and there is no direct access to the target within the tissue from the needle entry location (a potential obstacle may be located between the needle entry location and the target), the desired value for $\theta_{0}$ cannot be necessarily assumed a priori. This is because the needle motion inside soft tissue is constrained by its natural curvature. Nonetheless, for example, if this constraint is relaxed, then there is not any feasibility problem in reaching a target for a given initial location and $\theta_{0}$. The authors in [15] modeled the environment as a cubical region measuring $20 \mathrm{~cm}$ along each axis. Also, they considered a large needle entry region in their simulations, which in turn resulted in an increased possibility of needle motion in reaching a target for a given $\theta_{0}$. In our work, we mainly focused on a fixed needle entry location to reduce the tissue trauma incurred during the multi-target needle insertion procedure. Therefore, the assumption of considering a given $\theta_{0}$ is discarded in our algorithm, and, subsequently, a random value for $\theta_{0}$ is picked each time a random position in the configuration space is sampled. Finally, at the end of the algorithm, the correct value of $\theta_{0}$ is simply extracted from the planner.

Regarding sampling $\theta_{0}$, two scenarios can be considered. In the first case, once $\theta_{0}$ is set to a random value, it is kept constant for each time a random point in the configuration space is sampled. By choosing $\theta_{0}$ this way, the planner virtually defines the direction in which the tree is supposed to grow. Although there is some degree of maneuverability in needle motion, due to the limited value of the needle maximum curvature as well as the limited size of the needle workspace, it is almost impossible to compensate for the picked value of $\theta_{0}$, which is far from the right choice and, 
consequently, the planner returns failure. Table 4.2 shows the result of the planner's performance for the system configuration shown in Fig. 4.4 in which $\theta_{0}$ is randomly chosen just once during each algorithm execution.

Table 4.2: Numerical analysis of the performance of the RRT-based planner for different types of random sampling $\theta_{0}$, for each of which $\theta_{0}$ is set once during the algorithm execution. Two different target locations are considered for both of which $r=1 \mathrm{~mm}$ and $\kappa_{0}=0.2 \mathrm{~cm}^{-1}$. The results correspond to the mean values of $n=100$ simulations, where cost and time are in $\mathrm{cm}$ and $\mathrm{ms}$, respectively.

\begin{tabular}{|c|c|c|c|c|c|c|c|c|c|}
\hline \multirow{3}{*}{$\begin{array}{l}n= \\
100\end{array}$} & \multirow{3}{*}{$\begin{array}{l}\text { target } \\
\text { choice }\end{array}$} & \multicolumn{8}{|c|}{ type of sampling $\theta_{0}$} \\
\hline & & \multirow{2}{*}{$\begin{array}{l}\text { uniform } \\
\text { dist. }\end{array}$} & \multicolumn{7}{|c|}{ normal distribution $(\mu, \sigma)$} \\
\hline & & & $\sigma_{1}=10^{\circ}$ & $\sigma_{2}=20^{\circ}$ & $\sigma_{3}=30^{\circ}$ & $\sigma_{4}=40^{\circ}$ & $\sigma_{5}=50^{\circ}$ & $\sigma_{6}=60^{\circ}$ & $\sigma_{v}$ \\
\hline cost & \multirow{4}{*}{$T_{i o}$} & 8.80 & 8.65 & 8.70 & 8.73 & 8.72 & 8.75 & 8.73 & 8.72 \\
\hline time & & 242 & 64.7 & 92.8 & 101.2 & 233.4 & 241.1 & 247.4 & 173.4 \\
\hline$N_{n d s}$ & & 302.73 & 8.02 & 57.42 & 75.18 & 279.7 & 291.9 & 306.5 & 188.6 \\
\hline$N_{\text {fail }}$ & & 45 & 0 & 8 & 10 & 42 & 45 & 51 & 30 \\
\hline cost & \multirow{4}{*}{$T_{b o}$} & 10.53 & 9.82 & 9.93 & 9.97 & 10.16 & 10.15 & 10.16 & 10.04 \\
\hline time & & 201.4 & 48.6 & 45.4 & 59 & 59.6 & 74.6 & 98.3 & 66.7 \\
\hline$N_{n d s}$ & & 238.4 & 18.6 & 13.4 & 36.6 & 33.5 & 60.6 & 97.2 & 49 \\
\hline$N_{\text {fail }}$ & & 34 & 0 & 0 & 4 & 5 & 10 & 20 & 7 \\
\hline
\end{tabular}

In both uniform and normal sampling of $\theta_{0}$, the algorithm is biased toward the target. However, for normal sampling, two heuristics are added to the algorithm. For all the columns of normal distribution in Table 4.2, parameter $\mu$ is set to the tangent line connecting the needle initial location to the target. Nonetheless, different values are considered for standard deviation parameter $\sigma$ which starts from $\sigma_{1}=10^{\circ}$ to $\sigma_{6}=60^{\circ}$. The last column of the table refers to the case where parameter $\sigma_{v}$ is variable and chosen through uniform sampling of values between $10^{\circ}$ and $60^{\circ}$. The following implications can be deduced from Table 4.2.

1. As in the results of Table 4.1, the cost value is relatively robust with respect to changes in parameters. 
2. By adding two more biases to the algorithm, the planner's performance improves clearly. The improvement includes a smaller number of nodes in the tree and, consequently, less computation time, and a smaller number of the failures of the algorithm. By decreasing the $\sigma$ value, the improvement is more pronounced. Indeed, by narrowing the normal distribution (decreasing $\sigma$ ), the algorithm is narrowing the scope of sampling $\theta_{0}$ toward the ideal choice. On the contrary, increasing the $\sigma$ interval conducts the algorithm towards uniform sampling of $\theta_{0}$. In order to reduce the sensitivity of the algorithm to specific anatomy in terms of the relative location of the target and obstacle, a reasonable interval is chosen for $\sigma_{v}$.

3. As shown in Table 4.2, for most of the cases, the algorithm fails several times for every 100 simulations. On the other hand, in a complete version of the planning approach, as mentioned in the $\mathrm{ST}-\mathrm{RRT}_{2 \mathrm{D}}$ algorithm, the RRT algorithm is executed 50 times and, subsequently, an optimization is performed over a set of 50 results. Thus, any potential failure is removed from the set of outputs. Nevertheless, it would be worth our while to adapt sampling $\theta_{0}$ to remove any potential failure per each execution of the algorithm. This can directly enhance the planning efficiency.

Base on the above discussion, in the following, $\theta_{0}$ is sampled once at each iteration of the algorithm. Similarly, there are two options for sampling $\theta_{0}$, i.e. based on uniform distribution and normal distribution, as the new way of biasing $\theta_{0}$, which is used in our work. Table 4.3 shows the effect of biasing $\theta_{0}$ on the performance of the RRTbased planner for two different target locations in the system configuration shown in Fig. 4.4. Two types of biasing bias 2 and bias $_{4}$ are used. Regarding bias 2 , as explained in Subsection 4.2.2, the growth of the tree is biased toward the target by applying two heuristics [18]. Regarding bias $_{4}$, two more heuristics are added to bias 2 , which is 
to limit the height of the sampling point to the height of the target and to sample $\theta_{0}$ based on normal distribution whose standard deviation is randomly selected between $10^{\circ}$ and $60^{\circ}$ based on uniform distribution $\left(\sigma_{v}\right)$. Finally, when the tree reaches the target region, the path is extracted from the tree. However, concerning $\theta_{0}$, the right candidate can be computed from $P_{0}$ and the information of the path generated by the planner, which is the position and orientation data of the first node of the path.

Table 4.3: Numerical analysis of the performance of the RRT-based planner for a single target of different locations under two different ways of biasing the algorithm. The results correspond to $n=500$ simulations.

\begin{tabular}{|c|c|c|c|c|c|c|c|}
\hline \multicolumn{2}{|c|}{$n=500$} & \multicolumn{2}{c|}{ cost $(\mathrm{cm})$} & \multicolumn{2}{c|}{ time $(\mathrm{ms})$} & \multicolumn{2}{c|}{$N_{n d s}$} \\
\hline target choice & type of bias & mean & StDev & mean & StDev & mean & StDev \\
\hline \multirow{3}{*}{$T_{i o}$} & bias $_{2}$ & 8.71 & 0.14 & 53.68 & 9.66 & 17.64 & 19.65 \\
\cline { 2 - 8 } & bias $_{4}$ & 8.69 & 0.13 & 37.40 & 5.69 & 8.17 & 5.82 \\
\hline \multirow{3}{*}{$T_{b o}$} & bias $_{2}$ & 10.16 & 0.41 & 53.40 & 10.02 & 15.85 & 19.80 \\
\cline { 2 - 8 } & bias $_{4}$ & 9.99 & 0.39 & 39.27 & 7.23 & 11.27 & 10.13 \\
\hline
\end{tabular}

The following implications can be deduced from Table 4.3.

1. More importantly, there is no failure return at all.

2. As in the results of Table 4.1 and Table 4.2, the standard deviation for the cost values are low, which confirms the limited maneuverability of the needle motion.

3. The mean values of the computation time and the number of nodes in the tree are lower for both target locations in bias $_{4}$ which confirms the efficiency of the added heuristics in bias $_{4}$.

4. Concerning the standard deviations of time and $N_{n d s}$ for both target locations, 
the results of bias $_{4}$ are smaller than those for bias 2 which, through adding two more heuristics, confirms the branching of the tree is more objective. This can be verified in Fig. 4.4 as well.

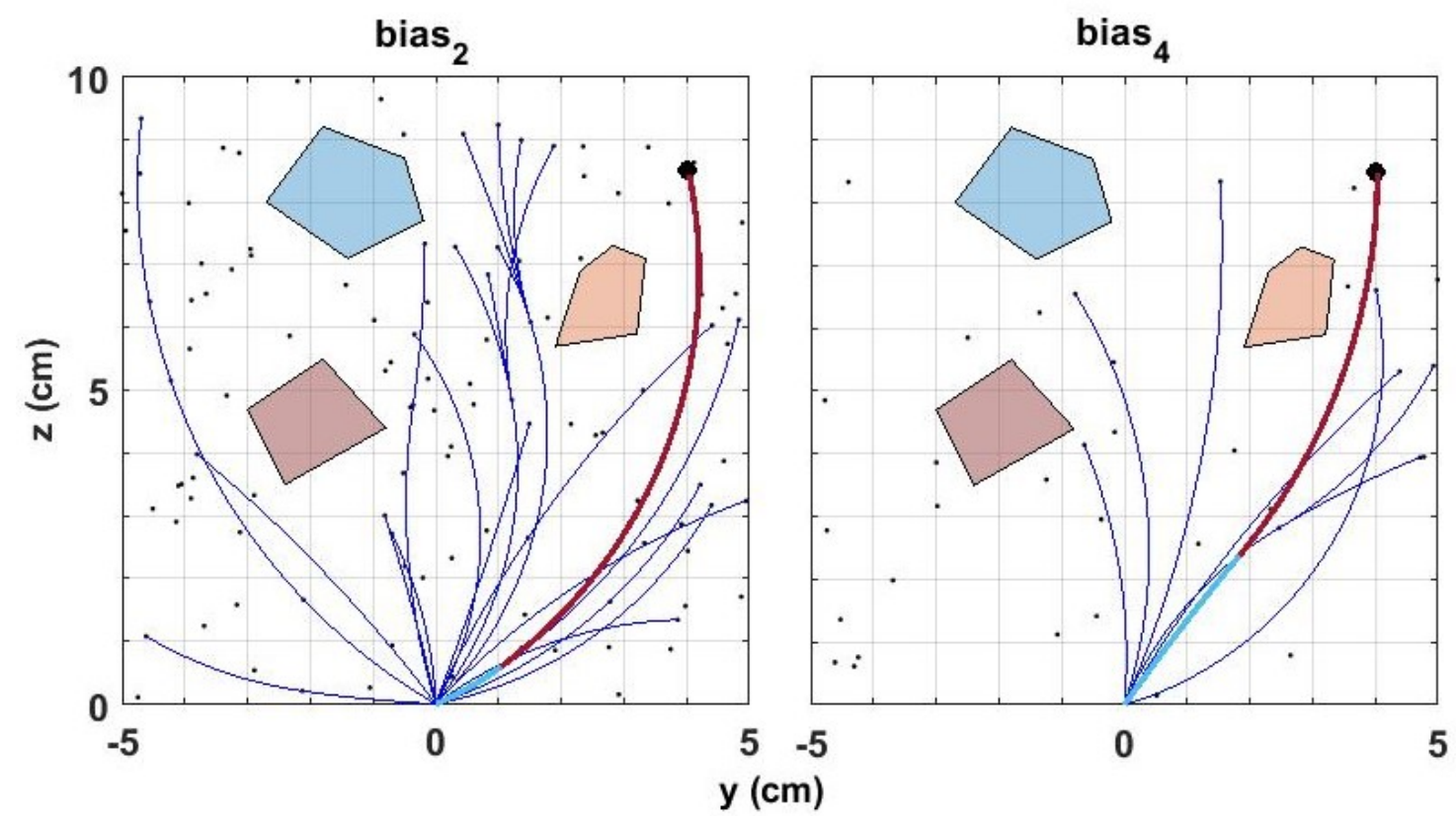

Figure 4.4: Comparison of the performance of the RRT-based planner for two types of biasing the algorithm, i.e., bias 2 and bias $_{4}$. The target is shown in black bullet $\left(T_{b o}\right)$.

As shown in Fig. 4.4, there are two different scenarios for a similar path-planning problem, with the only difference being in the way sampling is done, i.e. bias $_{2}$ and bias $_{4}$. For both plots, the path returned by the planner is depicted in bold in order to differentiate it from the other edges of the tree. As observed, the tree in plot $b i a s_{2}$ is expanded more uniformly in the configuration space with more nodes and, subsequently, more edges comparing to the plot in bias $_{4}$. The small dots in both plots refer to the sampled points that are not in the reachability region of any of the nodes in the tree at the time of sampling, and, therefore, they are not added to the tree. As can be deduced from Table 4.1 through Table 4.3 and Fig. 4.4, sampling $\theta_{0}$ based on normal distribution once each point in the configuration space is sampled 
and limiting the height of the sampled point to the height of the target result in fewer attempted samples before hitting the target, which, consequently, increases the planning efficiency.

After sampling $\theta_{0}$, from the initial state $X_{0}=\left[P_{0}, \theta_{0}\right]^{T}$ the planner incrementally builds $\mathfrak{T}$ toward $P_{\text {target }}$ while avoiding obstacles and complying with the nonholonomic constraints of the needle motion. At each iteration $k \leq K$, an obstacle-free position $P_{\text {rand }}$ is sampled from the free configuration space $\Re^{2}$. After sampling $P_{\text {rand }}$, the planner checks for feasible connectivity of $P_{\text {rand }}$ with the current vertices in the $\mathfrak{T}$ to construct the reachable set $Q_{\text {reach }}$. In this regard, for $P_{\text {rand }}$ and each vertex of $\mathfrak{T}$, the respective arc parameter $r_{i}$ is obtained through (4.1) and checked by $r_{0}=\kappa_{0}^{-1}$. To comply with the nonholonomic kinematics of the needle motion, $r_{i} \geq r_{0}$, or equivalently, $\kappa_{i} \leq \kappa_{0}$. If this is the case, the concerned vertex is added to $Q_{\text {reach }}$. In other words, $P_{\text {rand }}$ is in the reachability region of the concerned vertex. Therefore, $Q_{\text {reach }}$ represents the set of all vertices that $P_{\text {rand }}$ lies in their reachability region. Afterward, among the reachable set $Q_{\text {reach }}$, the planner finds the closest state to $P_{\text {rand }}$, denoted by $X_{\text {near }}=\left[P_{\text {near }}, \theta_{\text {near }}\right]^{T}$, based on a specific distance metric $\rho($.$) . As opposed to [15]$ with a Euclidean distance metric, here the arc length is chosen as the distance metric. Then the planner tries to expand the $\mathfrak{T}$ from $X_{\text {near }}$ toward the potential new state $X_{\text {new }}$, which has the same position as $P_{\text {rand }}\left(X_{\text {new }}=\left[P_{\text {rand }}, \theta_{\text {new }}\right]^{T}\right)$. $\theta_{\text {new }}$ can be obtained according to $\theta_{\text {new }}=\theta_{\text {near }}-\theta_{i}$, where $\theta_{\text {near }}$ is known and $\theta_{i}$ can be calculated from (4.2). Finally, if this connection is obstacle-free and inside the permissible workspace, $X_{\text {new }}$ and the corresponding arc segment connecting $X_{\text {near }}$ to $X_{n e w}$ are added to the $\mathfrak{T}$ as the new vertex and edge, respectively. This process goes on until either the $\mathfrak{T}$ is connected to $P_{\text {target }}$ or the maximum number of iterations is exceeded.

Once the needle has reached the target region, we used the breadth-first search (bfs) algorithm [82] to extract the plan from the constructed tree (See Appendix A for bfs algorithm). Thanks to fast computation of RRT planner, we iterate the 
planner 100 times in order to have a set of planned trajectories. Afterward, based on a clinician's specified criterion, which in our work is chosen to be the shortest path, the best plan can be selected as the solution. Although it is known that RRT planners do not provide optimality in a limited amount of time [27], iterating the planner for a certain number of times generates a high-quality path. To address the obstacle avoidance problem in the simulations, obstacles are modeled as polygonal shapes. In this regard, each circular arc segment is divided into 50 equally separated intervals. Then, any likely obstacle collision of each of these 50 locations is checked. Furthermore, to provide some robustness toward obstacle motion, a safety buffer is considered to be capable of providing proper clearance from the obstacles. Also, as explained in [23], needle buckling is one of the most prevalent complications in percutaneous needle interventions. To account for this issue, according to Reed et al. in [23], we limited the maximum change of the needle orientation in each circular arc segment to be less than $90^{\circ}\left(\theta_{i} \leq 90^{\circ}\right)$. The longer the curved bevel-tip needle is inserted, the greater the applied force by the needle against the tissue, helping the needle slice through the tissue which in turn causes needle buckling. Also, to increase the planning efficiency, we limit the height of the sampled point to be around the height of the target. The reason is obvious: as sampling points that are above the target require the needle to return to the target and the curvature of the needle is limited, this is almost impossible in clinical metrics. 


\subsection{Multiple-Target RRT-based Path-Planning Algorithm}

This section proposes a heuristic 2D RRT-based path-planning algorithm given its high computational speed in finding a high-quality path, which connects the needle's initial configuration toward the set of target locations in the minimum possible time for environments with obstacles. The current trend in operating rooms is to sequentially insert and retract the needle toward each separate target. In other words, after accessing the first target, the needle has to be retracted completely and then reoriented and reinserted toward the next target and proceeded with this procedure until all the accessible targets are reached. This is shown in Fig. 4.5. This method inevitably decreases procedure efficiency and increase tissue trauma. In view of the great amount of tissue damage in this approach, alternative approaches are preferred. Two common approaches are introduced in [73]. First, after the needle reaches the first target, without retracting the needle, try to reach the second target and this procedure continues until all the targets are reached. Instead of a tree-like path, the needle cuts through the tissue in a curved trajectory. Although it looks like a more reasonable and plausible approach, it has significant clinically imposed limitations. In view of the nonholonomic nature of the needle kinematics inside soft tissue, for a given workspace and at a given state it is not always feasible to reach the neighboring target in the configuration space, much less through minimum-length trajectories. In some special cases where the next target lies approximately in the same direction of the current needle tip's orientation, it may be possible. However, in other cases, it is necessary for the needle to be retracted partially from the currently reached target and then inserted toward the next target. This leads to the second approach, in which needle retraction is allowed. Our method is based on the second clinically-relevant approach. 


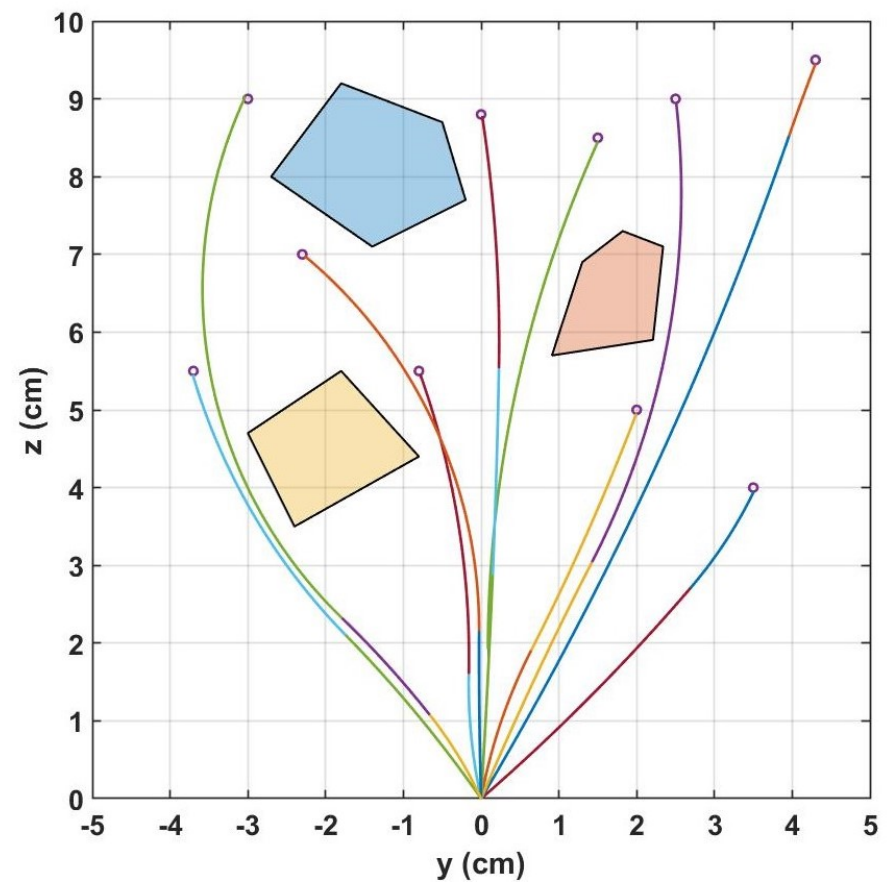

Figure 4.5: Multi-target planning includes individual RRT-based planning for each target. As a result, there are as many sequential insertions and retractions of the needle as the number of the targets.

\subsubsection{Clinical Relevance of the Algorithm}

A key point in steerable needle trajectory planning is its clinical relevance and practicality through a proper choice of system parameters [83]. It is known that needle trajectory during its motion in biological tissue is affected by its natural curvature [14] and using the DC method [58] could only help to generate variable curvatures up to needle natural curvature. Intuitively, the larger the needle natural curvature $\left(\kappa_{0}=\right.$ $\left.1 / r_{0}\right)$, the more maneuverability the needle has during its motion. This consequently results in a greater possibility of accessing any neighboring target. However, obtaining a clinical needle with large $\kappa_{0}$ is challenging. Different works have addressed the singletarget path-planning problem of flexible bevel-tip needles with a radius of curvature equal to $6 \mathrm{~cm}$ [13], [76], [80], [84] and $5 \mathrm{~cm}$ [67], [85]. However, these works are based 
on nitinol wires, which are incapable of delivering load to the predefined locations inside soft tissue. Adebar et al. in [86] observed that in order to reach the majority of the liver organ, the minimum radius of curvature below $5 \mathrm{~cm}$ is required. Unlike the previous work [87], which had tried to obtain a similar radius of curvature but with nitinol wires of a $0.5 \mathrm{~mm}$ diameter, they designed a clinically relevant articulated-tip steerable needle capable of both delivering a similar radius of curvature as well as providing practicality in delivering payloads such as fluids and ablation electrodes to the target. Moreover, their needle can pass through the liver sheath and different layers and membranes. As explained in [71], the path-planning methods that generate complicated paths with many turns during needle motion are not practical choices for the needle in a clinical application. Strictly speaking, to realize a clinically consistent method, the correct choice of workspace dimensions is an important factor to be accounted for in the planner. The work in [73] modeled the environment as a unit square and the minimum radius of curvature was assumed to be 0.1 unit. As a result, in order to realize a clinically relevant needle trajectory, the square has to be of half a meter long on each side, which is much greater than real dimensions. The value of the needle radius of curvature $5 \mathrm{~cm}$ alone is a serious limiting factor in the needle's motion in terms of accessing a neighboring point from a given state in a given clinical workspace. As a result, we focus on the multi-target path-planning problem where retraction is permissible.

It has been observed that by partially retracting the needle inside soft tissue, it follows the same path that the needle has cut through before retraction [23]. Therefore, inserting the needle from the initial configuration toward a target, and then, partially retracting, reorienting, and reinserting it toward the next neighboring target until all targets are reached can go a long way toward mitigating tissue damage. This mitigation in damage is down to a reduction in the needle inserted length. With partial retraction, the final needle trajectory takes a tree-like path instead of a curved one. 
As shown by Reed et al., in [23], through integrating three independent controllers, namely a 2D motion planner [67] that guides the needle around obstacles to reach a target in a desired plane, a planar controller [88] that maintains the needle in the desired plane, and a torsion compensator [21] that controls the needle tip orientation about the needle shaft, a real-world 3D robotic-assisted needle-steering system can be achieved.

Besides the main difference between the two above-mentioned approaches (where retraction is admissible and inadmissible) in view of practicality in clinical metrics, another source of difference emanates from different costs associated with each method. Totally, the cost $J$ is usually formulated in such a way that it ensures less tissue damage as well as provides some robustness in needle tip's minimum clearance from the obstacles during the total insertion time $T$. The weighted summation of these two objectives, $J$, can be formulated as follows.

$$
J(v, \omega, T)=\alpha_{i} \int_{0}^{T} J_{i}(f(v(t))) d t+\frac{\alpha_{o}}{T} \int_{0}^{T}\left(-J_{0}\left(d_{o}(t)\right)\right) d t
$$

In (4.4), $d_{o}$ is the distance of the needle tip from the closest obstacle. $J_{i}$ in the first term in (4.4) in general reflects the costs associated with both needle insertion and retraction and the function $f$ could differ for either of them, while $J_{o}$ in the latter term refers to the costs associated with obstacle collision. Depending on the application at hand, the trade-off between these two terms can be adjusted by a proper choice of $\alpha_{i}$ and $\alpha_{o}$, accordingly. Due to the assumptions made in [14], the tissue does not significantly deform during the needle's motion inside soft tissue. Consequently, during needle partial retraction, the needle follows the same path as it is cut during insertion and, therefore, it is reasonable to assume that there is a negligible cost involved during retraction intervals. In our work, we set $\alpha_{o}=0$ and only focus on the needle inserted length in the interests of minimizing the costs. To address the obstacle 
avoidance problem, as in the single target algorithm, each circular arc segment is divided into 50 equally separated intervals. Then, any likely obstacle collision of each of these 50 locations on the circular arc segment is checked. Furthermore, to provide some robustness toward obstacle motion, a safety buffer is considered by fattening the polygonal shapes to be capable of providing proper clearance from the obstacles.

Our Multi-Target RRT-based Planning Algorithm is outlined in MT-RRT 2 . The input to $\mathrm{MT}-\mathrm{RRT}_{2 \mathrm{D}}$ is the same as the one to ST-RRT $2 \mathrm{D}$; however, instead of a single target we have a set of targets defined as $S_{T}$. Regarding the outputs, instead of a single curved trajectory as in $\mathrm{ST}-\mathrm{RRT}_{2 \mathrm{D}}$, here in $\mathrm{MT}-\mathrm{RRT}_{2 \mathrm{D}}$, we have a tree-like trajectory that connects $P_{0}$ to $S_{T}$. 


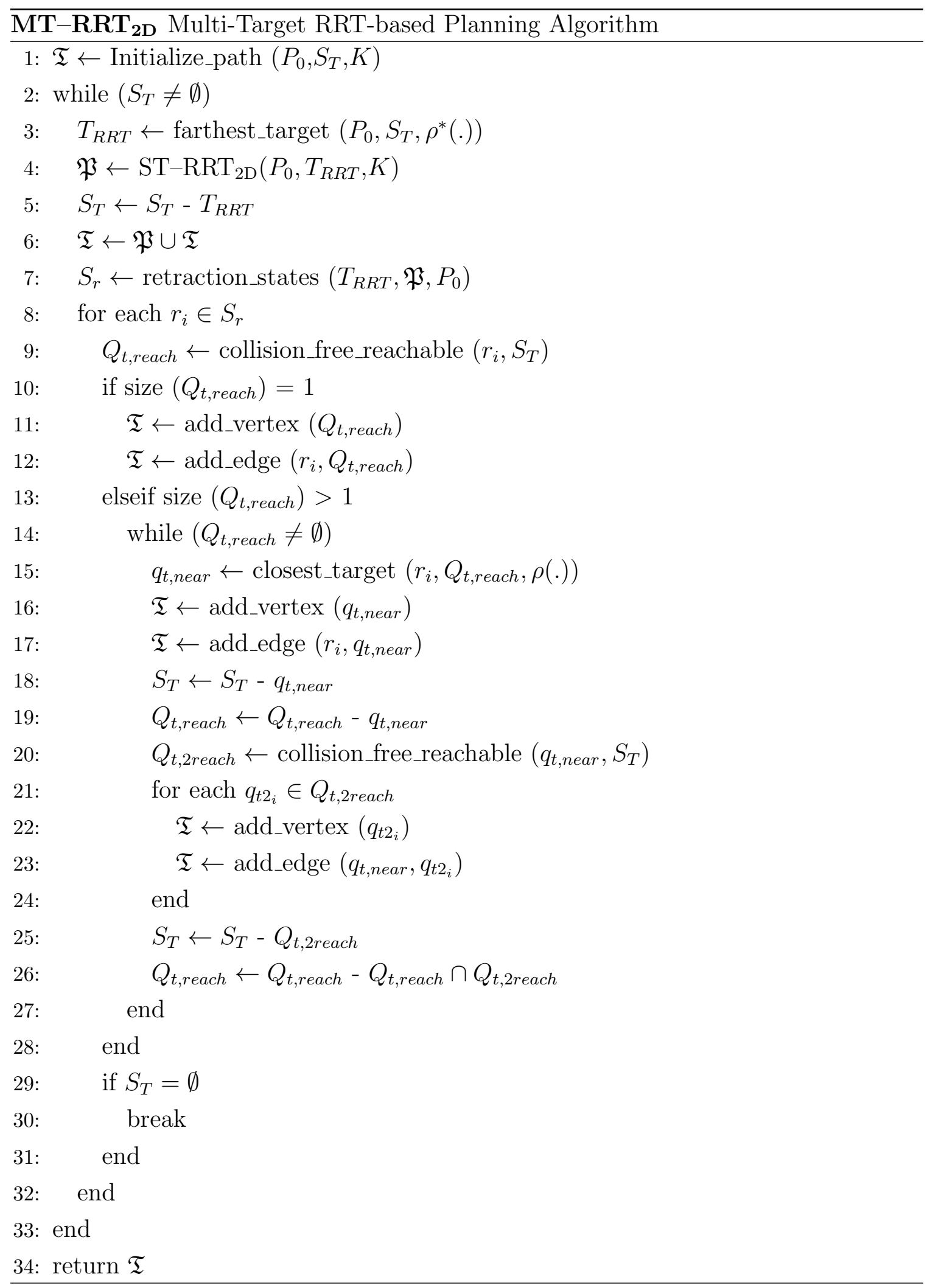


As described in MT- $\mathrm{RRT}_{2 \mathrm{D}}$, to begin with, based on the Euclidean distance metric $\rho^{*}($.$) , the planner selects the farthest target as the first target T_{R R T}$ to execute the conventional RRT planning algorithm, as explained in $\mathrm{ST}-\mathrm{RRT}_{2 \mathrm{D}}$. The RRT planner calculates the shortest path among the available candidates as a high-quality solution $\mathfrak{P}$, which will be used during the needle retraction phase to access the other targets. Each time a target is visited, it is removed from $S_{T}$; therefore, $S_{T}$ represents the set of targets not reached yet. In the case of no solution to the RRT planner for the single target $T_{R R T}(\mathfrak{P}=\emptyset)$, instead of terminating the whole process, the almostunreachable target is removed from $S_{T}$ and the planning process continues with the rest of the unvisited targets.

After accessing the first target, the needle retracts along RRT-planned trajectory $\mathfrak{P}$ incrementally up to the root node. In doing so, the planner calculates a certain number of states, called retraction states, which are equally spaced per circular arc segment. In fact, a retraction state is a location in the free configuration space where the needle might stop retracting on the RRT-planned trajectory and be inserted toward the other targets. We define the set of retraction states, starting with $T_{R R T}$ and finishing with $P_{0}$, as $S_{r}$, where $S_{r} \in \mathfrak{P}$. At each retraction state $r_{i} \in S_{r}$, the planner checks the collision-free reachability of all the unvisited targets $S_{T}$ through function collision_free_reachable in $\mathrm{MT}-\mathrm{RRT}_{2 \mathrm{D}}$, where the respective collision-free reachable set is denoted by $Q_{t, \text { reach }}$. It is worth noticing that besides constraining each circular arc length through bounding $\theta_{i}$ to be less than $90^{\circ}$ to prevent buckling, the length of each circular arc is further limited through this function. The reason is to exclude the lengthy arcs from being added to $\mathfrak{T}$ with the hope of being visited through shorter paths by the rest of the algorithm. If there is a single collision-free reachable target in $Q_{t, \text { reach }}$ at the current retraction state $r_{i}$, the planner connects that target to $r_{i}$ through a single curve; otherwise, if nothing is found while $S_{T} \neq \emptyset$, the algorithm proceeds to the next retraction state $r_{i+1}$. In the case of more than one collision-free 
reachable target at each retraction state $r_{i}$, the planner first checks for the closest target $q_{t, \text { near }}$ in $Q_{t, \text { reach }}$, where the arc length is chosen as the distance metric $\rho($.$) .$ Then, the planner connects $q_{t, \text { near }}$ to the current retraction state $r_{i}$ through a single curve. Each time a target in $Q_{t, \text { reach }}$ is reached, it is removed both from $S_{T}$ and $Q_{t, \text { reach }}$ to update $Q_{t, \text { reach }}$ as the set of collision-free reachable targets from $r_{i}$, not reached yet.

Subsequently, we added an extra level of examining the collision-free reachability for all the unvisited targets $S_{T}$ from $q_{t, n e a r}$. This set of collision-free reachable targets from $q_{t, \text { near }}$ is defined by $Q_{t, 2 \text { reach }}$. The planner sequentially connects each $q_{t 2_{i}} \in$ $Q_{t, 2 \text { reach }}$ to $q_{t, \text { near }}$ through a single-curve trajectory. In other words, at each stage, the needle is inserted towards a state $q_{t 2_{i}}$ from $q_{t, \text { near }}$ and, it is, then, retracted toward $q_{t, n e a r}$. This procedure continues till all the targets $q_{t 2_{i}} \in Q_{t, 2 \text { reach }}$ are visited by the planner. It should also be noted that there might exist some common states between $Q_{t, \text { reach }}$ and $Q_{t, 2 \text { reach }}\left(q_{t 2_{i}} \in Q_{t, \text { reach }} \cap Q_{t, 2 \text { reach }}\right)$. Therefore, $Q_{t, \text { reach }}$ has to be updated by removing that common state. Since the reachability test is nothing just a trigonometric relationship, this extra level improves the planner's efficiency. Moreover, in cases where two targets can be reached by almost one single circular arc segment, this added level eliminates the need for passing through the same arc two times, which reduces the cost of the procedure. This issue is depicted in Fig. 4.6. As shown, target $T_{4}$ is reached from target $T_{3}$. Therefore, instead of retracting the needle after reaching target $T_{3}$, the needle is navigated toward the next target $T_{4}$ from $T_{3}$, which clearly results in a reduced cost for the whole needle insertion procedure. In case of no collision-free reachable target from $q_{t, n e a r}$, the needle retracts toward the current retraction state $r_{i}$. The planning procedure continues till all targets belonging to $Q_{t, r e a c h}$ are accessed. If there are still targets that have yet to be visited, a similar procedure is done at the next retraction state $r_{i+1}$; this trend continues till it reaches the root node. In case of $S_{T} \neq \emptyset$, the algorithm is repeated with the set 


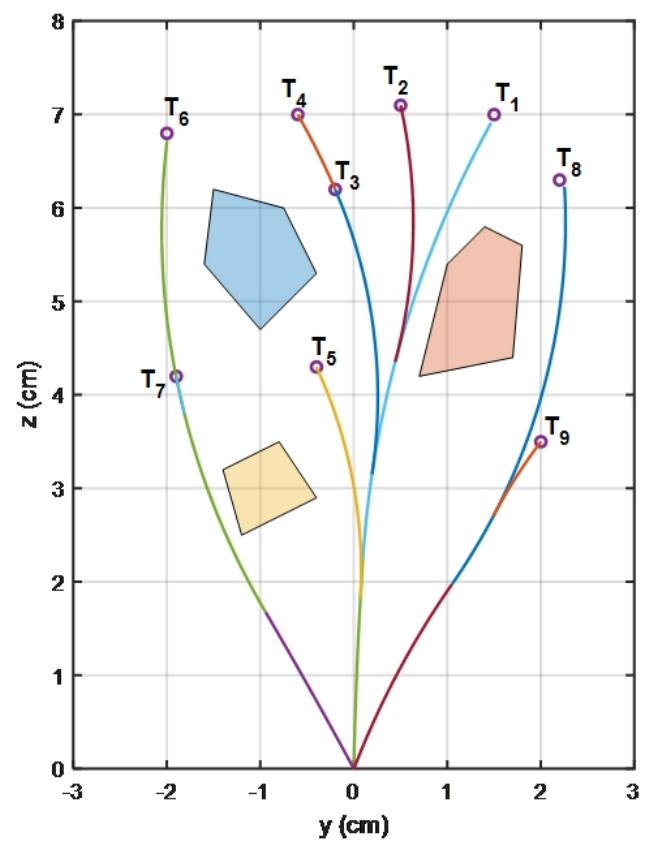

Figure 4.6: Planner's efficiency in reaching targets $T_{3}$ and $T_{4}$, which are almost in the same direction of the needle bevel tip; after reaching target $T_{3}$, without necessarily having to retract, the needle is navigated toward $T_{4}$.

of unvisited targets $S_{T}$. Again, first, among the unvisited nodes in $S_{T}$, the planner finds RRT-based Path $\mathfrak{P}$ for the closest unvisited target $T_{R R T}$ and, subsequently, retracts on this path incrementally to access the reachable targets. As mentioned earlier, the whole planning procedure consists of two phases: (I) reaching target $T_{R R T}$ through RRT-planned trajectory $\mathfrak{P}$ and (II) retracting on path $\mathfrak{P}$ to access the rest of reachable targets. The algorithm continues until all the targets are visited, which could be either reached or declared as almost impossible to reach. To exemplify the algorithm, as shown in Fig. 4.6, the whole planning process consists of three RRTplanned trajectories $\mathfrak{P}$, each of which connects $P_{0}$ to $T_{R R T}$. The first $\mathfrak{P}$ connects $P_{0}$ to $T_{R R T}=T_{1}$, the second $\mathfrak{P}$ connects $P_{0}$ to $T_{R R T}=T_{6}$, and the last and third $\mathfrak{P}$ connects $P_{0}$ to $T_{R R T}=T_{8}$. Each time, after reaching $T_{R R T}$, the retraction phase starts and the needle reaches all the reachable targets during the retraction phase, 
which for the first $\mathfrak{P}$, the needle reaches targets $T_{2}$ through $T_{5}$, while for the second $\mathfrak{P}$, the needle reaches target $T_{7}$, and for the last $\mathfrak{P}$, the needle reaches target $T_{9}$.

\subsubsection{Experimental Results}

We implemented our motion planner in Matlab R2018a and tested it on a $3.6 \mathrm{GHz}$ Core i7 CPU. We set the needle natural curvature to be $\kappa_{0}=0.2 \mathrm{~cm}^{-1}$. First, according to [58], [67], [85], [89] we set the workspace dimensions and needle entry location $P_{0}$ as $10 \times 10 \mathrm{~cm}^{2}$ and $[0,0]^{T}$, respectively to address the path-planning problem in prostate brachytherapy. Fig. 4.7 shows the tree-like planned trajectory by our new multi-target planner for the needle to reach targets $T_{1}$ through $T_{10}$. The planning

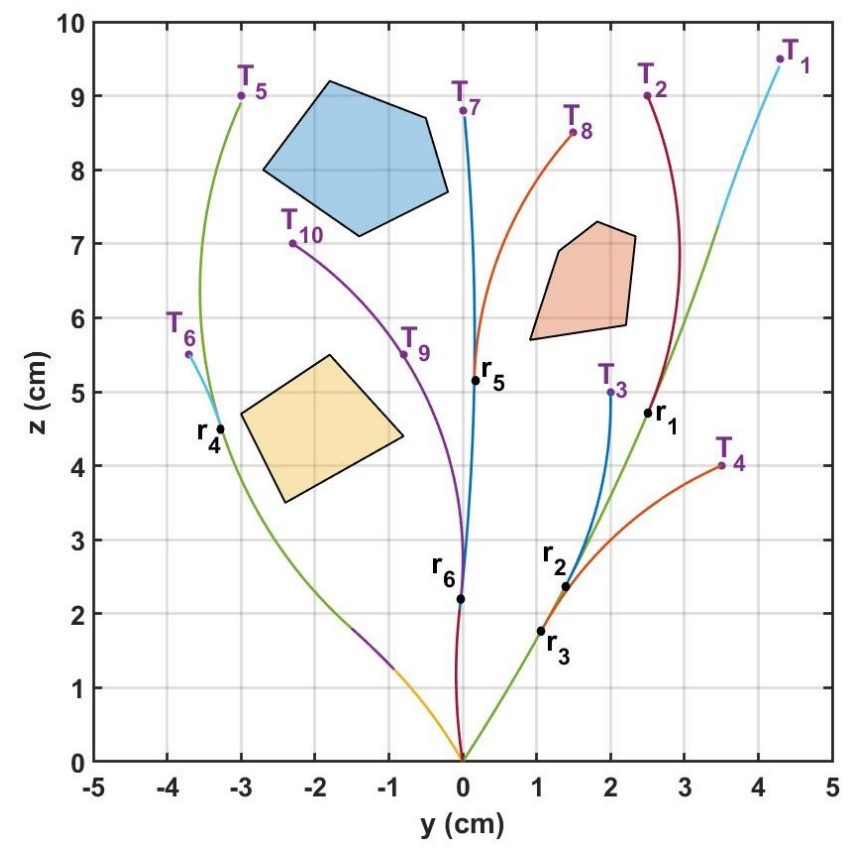

Figure 4.7: Multi-target planning under the novel RRT-based planner. The retraction states are tagged by small black bullets. The subscripts for both the targets and retraction states represent the order in which each is reached.

process combines the single target RRT-based planning algorithm, described in ST$\mathrm{RRT}_{2 \mathrm{D}}$, with collision-free reachability tests along the planned trajectory during the needle retraction phase. As shown in Fig. 4.7, first, $T_{1}$ is selected as $T_{R R T}$. Then, the 
planner performs the ST-RRT 2 D Algorithm to reach $T_{1}$. After accessing $T_{1}$ through path $\mathfrak{P}$, the needle is retracted incrementally along this path till retraction state $r_{1}$. At $r_{1}$ the needle is inserted toward the next target $T_{2}$, which is the only collision-free reachable target from $r_{1}$. Since there is no more reachable target at $T_{2}$, the needle is retracted from $T_{2}$ till $r_{1}$. Retraction along path $\mathfrak{P}$ continues till the retraction state $r_{2}$, where the only reachable target is $T_{3}$. After reaching $T_{3}$, the needle is retracted on the recent curve till $r_{2}$. A similar procedure is considered for $r_{3}$ and $T_{4}$. While the needle is retracted till the needle entry position, the whole above-mentioned procedure is repeated for targets $T_{5}$ and $T_{6}$ and retraction state $r_{4}$. For the second time, the needle is inserted from $P_{0}$ to reach the farthest unvisited target $T_{5}$ and later retracted incrementally till the needle entry position, while during the retraction procedure and at retraction state $r_{4}$, the needle is navigated toward target $T_{6}$. For the third and last time, a similar procedure is reiterated for targets $T_{7}$ through $T_{10}$ and retraction states $r_{5}$ and $r_{6}$. Later, since all the targets have been visited and reached, the algorithm is terminated and the needle is retracted completely. A rather close comparison of Fig. 4.5 with Fig. 4.7 clearly shows that through using our new multi-target RRT-based planning algorithm in Fig. 4.7, the costs associated with the needle insertion procedure decrease substantially and, as a result, there is less tissue damage during the procedure. The results of comparing the cost and computation time for our new RRT-based planner in Fig. 4.7 and the traditional one in Fig. 4.6 are shown in Fig. 4.8, which attest to a significant improvement when our new planner is used. There could be some special cases during a general needle insertion procedure where a target or a set of targets is not reachable. Fig. 4.9 shows the case where the target is both so close and right behind the obstacle. Therefore, the planner fails to reach the target due to the nonholonomic constraint of needle motion and not the planner's inefficiency. In this case, the planner puts aside the inaccessible target and continues the process with the remaining targets. 

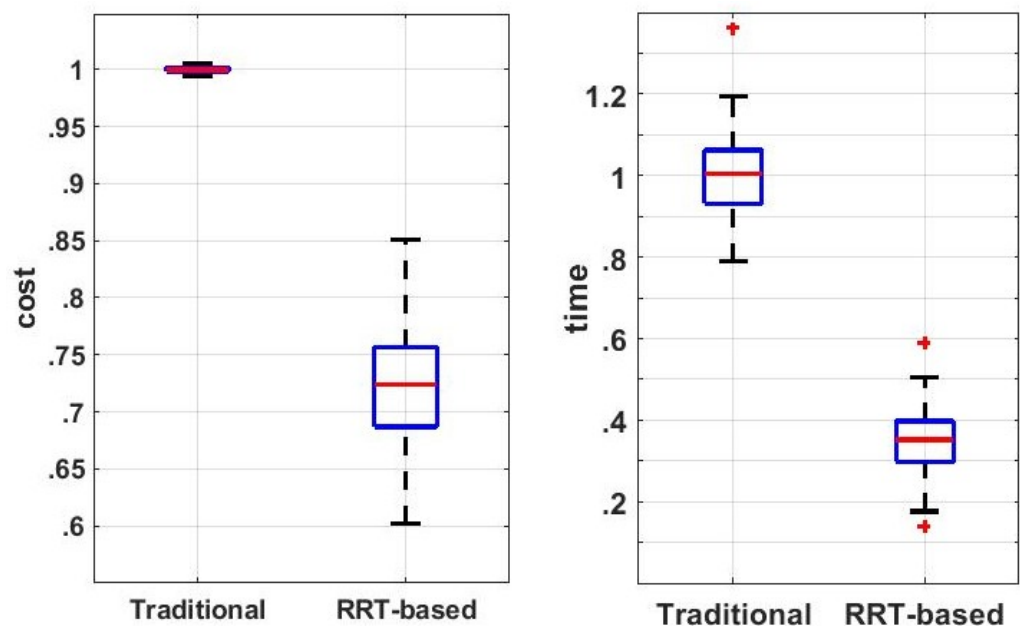

Figure 4.8: Comparison of our new multi-target RRT-based planner with the traditional one for the system configuration shown in Figs. 4.5 and 4.7. The cost and time data are normalized with respect to the corresponding mean value of the traditional planner. The results correspond to $n=100$ simulations.

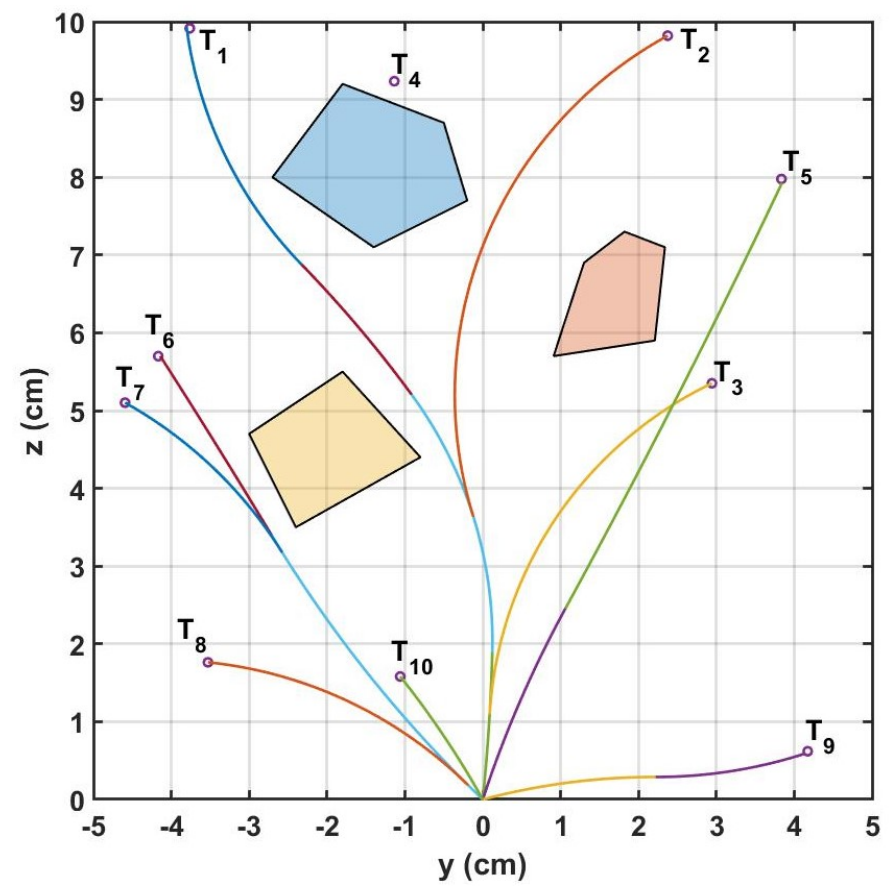

Figure 4.9: The planner is unable to visit all the 10 randomly-chosen targets $T_{i}, i=1, \ldots, 10$. Regarding $T_{4}$, the planner fails in view of the nonholonomic constraints of needle motion and limited real clinical metrics. 
To address the issue of workspace size in different applications, according to [90-92], for liver biopsy and [83] for breast biopsy, we picked two different sizes of workspaces in the simulations, namely, a small and a large workspace with dimensions of $5 \times 7.5 \mathrm{~cm}^{2}$ and $10 \times 15 \mathrm{~cm}^{2}$, respectively. Fig. 4.10 shows the performance of our new RRT-based planner to reach multiple targets in two different sizes of workspaces. Since, we define the larger workspace by double sizing of the smaller one, the cost of needle insertion procedure is almost doubled for the larger workspace.

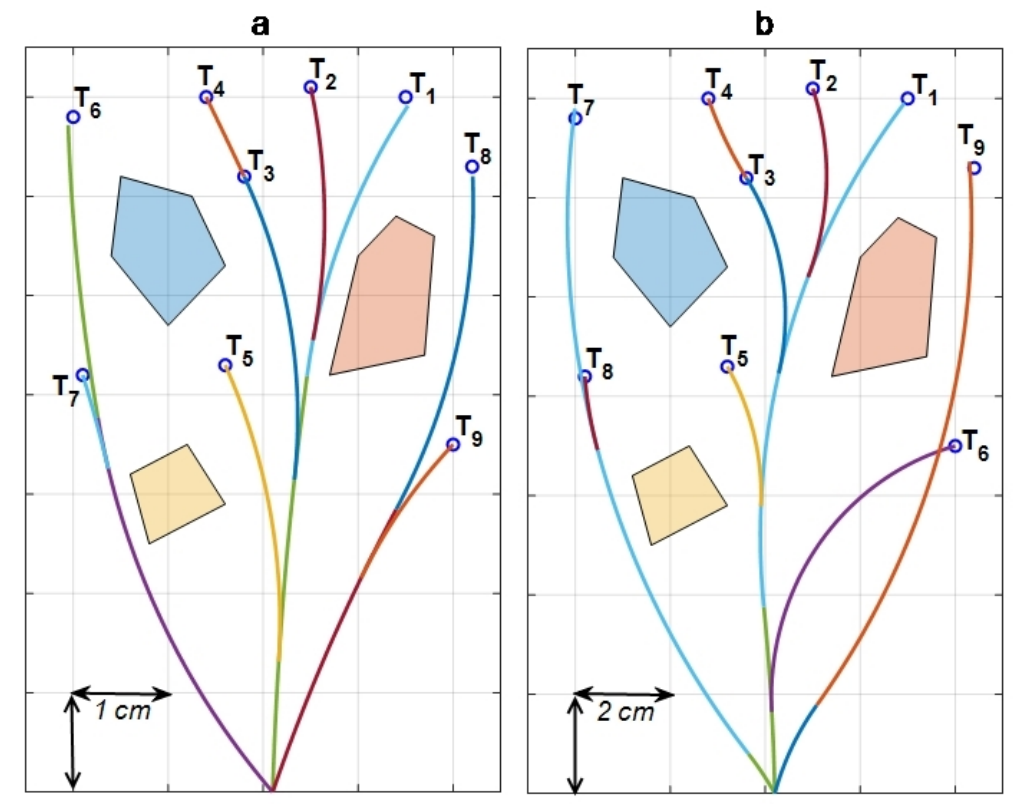

Figure 4.10: The novel multi-target RRT-based planner for two sizes of workspaces: a) the smaller workspace, b) the larger workspace with doubled dimensions of the smaller one.

It is clearly observed that the constructed tree is composed of more curved trajectory segments for the larger workspace, which is due to the increased maneuverability of the needle motion. Table 4.4 provides a numerical comparison of the results obtained through our new multi-target RRT-based planning approach and the traditional approach which is based on sequential insertion and retraction of needle for each separate target. As shown, in both the small and the large workspaces the time and 
Table 4.4: Numerical analysis to compare the performance of our new multi-target RRT-based planner with that of the traditional planner for both the small and large workspaces. The results correspond to $n=100$ simulations.

\begin{tabular}{|l|c|c|c|c|c|c|c|c|}
\hline \multirow{2}{*}{$n=100$} & \multicolumn{3}{|c|}{ New RRT-based Planner } & \multicolumn{3}{c|}{ Traditional Planner } \\
\cline { 2 - 9 } & \multicolumn{2}{|c|}{ cost $(\mathrm{cm})$} & \multicolumn{2}{c|}{ time $(\mathrm{ms})$} & \multicolumn{2}{c|}{ cost $(\mathrm{cm})$} & \multicolumn{2}{c|}{ time $(\mathrm{ms})$} \\
\cline { 2 - 9 } & mean & StDev & mean & StDev & mean & StDev & mean & StDev \\
\hline small & 35.89 & 2.13 & 361.49 & 87.75 & 54.06 & 0.10 & 979.08 & 107.85 \\
\hline large & 70.10 & 3.64 & 133.56 & 56.05 & 108.66 & 0.28 & 544.32 & 62.57 \\
\hline T-Test & \multicolumn{3}{|c|}{$3.2818 \mathrm{E}-149$} & $8.91659 \mathrm{E}-71$ & \multicolumn{2}{|c|}{0} & $3.20032 \mathrm{E}-89$ \\
\hline F-Test & \multicolumn{3}{|c|}{$8.52921 \mathrm{E}-06$} & \multicolumn{3}{|c|}{0.187683575} & \multicolumn{3}{c|}{$7.16051 \mathrm{E}-20$} & \multicolumn{3}{c|}{$4.56425 \mathrm{E}-06$} \\
\hline
\end{tabular}

the cost associated with the needle insertion is decreased considerably using our new RRT-based planner. The average reduction in cost for both the small workspace and the large workspace is $35 \%$. The average processing time of the algorithm in the small and large workspaces is $0.36 \mathrm{~s}$ and $0.13 \mathrm{~s}$, respectively, which according to [23], [18], is the required time interval (less than 1s) for the intraoperative control of the needle insertion system. Moreover, the statistical results of $\mathrm{T}-$ Test and $\mathrm{F}-$ Test reveal the difference between the small and large workspaces for both the new and the traditional planners. Along with the workspace dimension issue, it is important to note that in both workspaces shown in Fig. 4.10, the targets are scattered in a rectangular area, where the ratio of the vertical dimension to the horizontal dimension is 1.5. However, by increasing the horizontal dimension, this ratio decreases and, accordingly, the deviation of the targets in the horizontal direction increases. Consequently, the effectiveness of our new planner in terms of reducing the cost associated with needle insertion decreases as well. This can be verified by comparing the results shown in Fig. 4.8, where the reduction in cost is less than the cost associated with the results presented in Table 4.4 (related to Fig. 4.10). This is because based on the limited 
maneuverability of needle motion, for workspaces with greater horizontal dimensions, the needle has to retract for a longer distance to be able to reach the next target and, subsequently, to pass through a longer insertion distance for that target. On the other hand, when the targets are scattered more in vertical dimensions, the needle can reach other targets through a shorter insertion length and without having to be retracted significantly. It should also be noted that in Fig. 4.8 and Table 4.4, the standard deviations associated with the cost of the new multi-target RRT-based planner for three different sizes of the workspace are greater comparing to the sequential planner. This is due to the freedom of the new multi-target RRT-based planner in choosing the order in which targets are reached. For example, by running the algorithm at different times, the order in which a specific target is reached may vary, which could directly lead to a different output of the planner, and subsequently, a different cost.

\subsection{Importance of the DC approach}

The significance of the DC approach described in Section 3.4, cannot be overemphasized. Instead of choosing a constant curvature path, by considering $0 \leq \kappa \leq \kappa_{0}$, the DC approach makes different curvatures of trajectories possible. On the other hand, limiting the path curvature inevitably reduces the planner's efficiency. To illustrate the critical effect of the path curvature, we limit it to be lower bounded such as $\kappa_{\min } \leq \kappa \leq \kappa_{0}$. Equating $\kappa_{\min }=0$ results in the DC approach, while, on the other hand, setting $\kappa_{\min }=\kappa_{0}$ is equivalent to having constant curvature paths of curvature $\kappa_{0}$, which is the most limiting case in path planning. Therefore, by approaching $\kappa_{\min }$ from 0 to $\kappa_{0}$, the planner's efficiency drops significantly. This concept is illustrated in Fig. 4.11 and Fig. 4.12. As can be observed, as $\kappa_{\min }$ increases, the costs and the planning time increase for both the small and the large workspaces. Interestingly, the degradation in planner's performance for the smaller workspace is 
more pronounced due to the rather poor maneuverability of the needle motion inside smaller workspaces. Moreover, exponential increase in simulation run time in Fig. 4.12 , as a result of increasing $\kappa_{\min }$ makes the planner an inappropriate candidate for intraoperative applications.
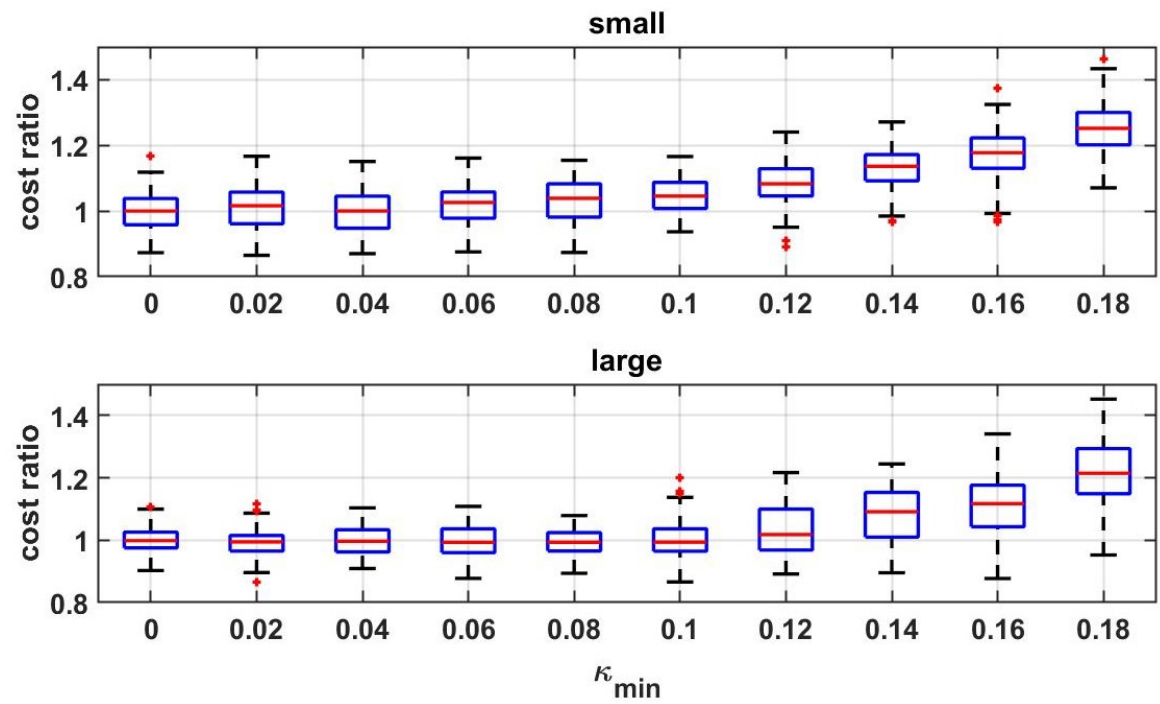

Figure 4.11: The ratios of the costs associated with the needle insertion procedure are compared for different values of $\kappa_{\min }$ for both the small and the large workspaces. The result for each value of $\kappa_{\min }$ corresponds to 100 simulations. All the data are normalized with respect to the cost mean value of our new planner where $\kappa_{\min }=0$.

\subsection{Summary}

In this chapter, we described our novel 2D RRT-based planning algorithm for a single target as well as multiple targets at great length. Regarding the preoperative single-target 2D RRT-based path-planning problem, different works in the literature discuss planner's satisfactory performance under some given assumptions. In particular, needle initial orientation $\theta_{0}$ is considered as a known parameter obtained through preoperative medical images. In our work, we relaxed this assumption by 

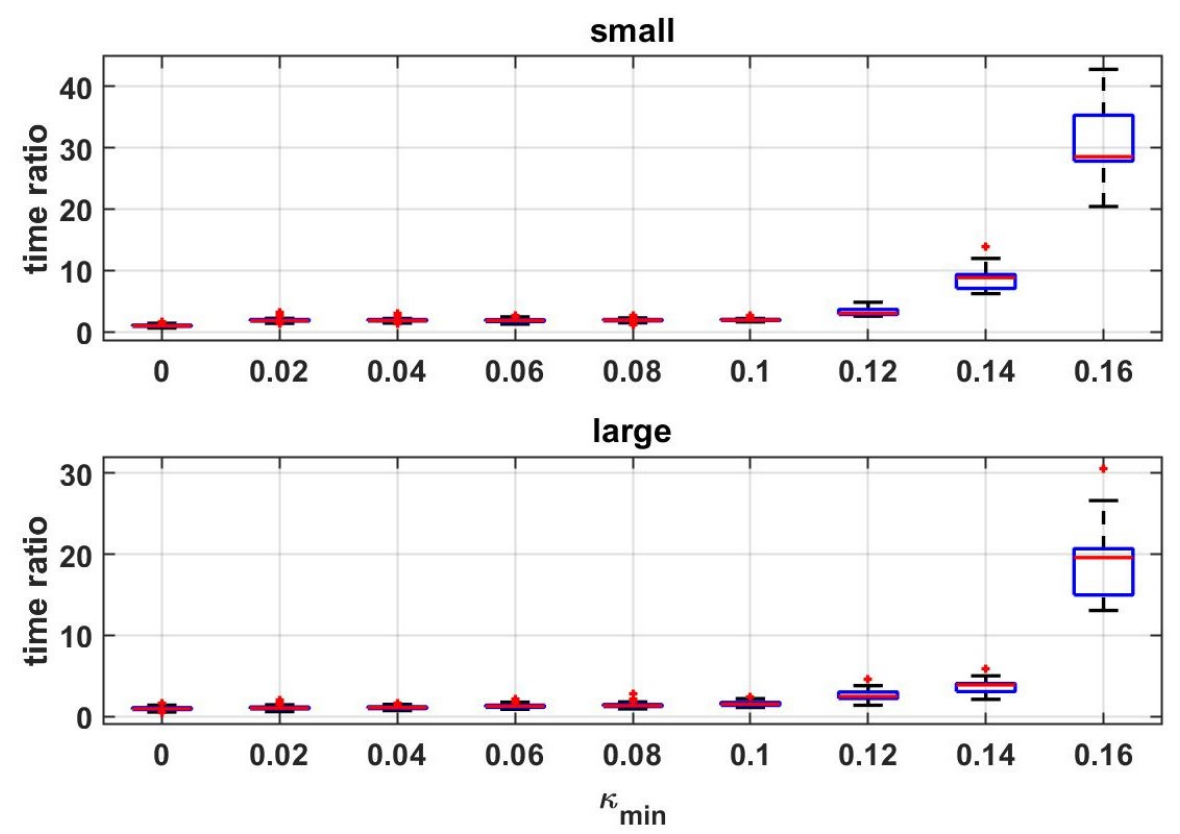

Figure 4.12: The ratios of the simulation run time are compared for different values of $\kappa_{\text {min }}$ for both the small and the large workspaces. The result for each value of $\kappa_{\text {min }}$ corresponds to 100 simulations. All the data are normalized with respect to the time mean value of our new planner where $\kappa_{\text {min }}=0$. To provide a clearer graph, the results for the marginal value of $\kappa_{\min }=.18$ are not presented. The ratios corresponding to $\kappa_{\min }=.18$ are 157 and 145 for the small and the large workspaces, respectively. This abrupt increase in the simulation run time reveals the noticeable degradation in the planner's efficiency while using almost constant-curvature trajectories.

considering $\theta_{0}$ as an unknown variable, which is calculated by the planner. Moreover, we added two more heuristics to the RRT planning algorithm in order to increase its efficiency. Several simulation results have been provided to verify the efficiency of the proposed planner. Concerning the preoperative multi-target 2D RRT-based path-planning problem, our planner is able to reach as many targets as required in an environment with obstacles, where the needle has to go through narrow passages within the tissue to avoid collision with any potential obstacle. Despite some works considering system parameters excessively large, as the core element in our planning 
algorithm, we focused on the clinically-relevant choice of parameters including the needle natural curvature, workspace dimensions, target region, and needle entry location. The simulations provided in this chapter confirm that our planner works well under practical applications where real-world dimensions and metrics are chosen for the system. In contrast to global optimization-based methods, our planner works well independently of the number of targets and system complications. More importantly, the total tissue trauma experienced through our planning approach decreases significantly as compared to the traditional method, which is being currently used in operating rooms. In view of the ever-increasing interest in visual-based measurement [93], our planner can be integrated into a real-time needle tracking system. Consequently, by performing fast path re-planning at specific time intervals using real-time imaging data, the intraoperative control of the multi-target needle insertion system can be achieved. 


\section{Chapter 5}

\section{D Real-Time RRT Path Planner for Steerable Bevel-Tip Needles to Reach Multiple Moving Targets in a Simulated Real-World Application}

\subsection{Introduction}

A real-world needle insertion procedure cannot be anticipated perfectly beforehand since there is always a certain degree of uncertainty in needle-tissue interaction, and possible disturbances are likely to occur during needle insertion. A biological tissue is inhomogeneous with a varying stiffness property. Also, due to tissue deformation during needle insertion, the target and potential obstacles may move. Any disturbances in needle motion may be observed due to real-world uncertainties such as patient breathing or any unexpected physiological changes. The works discussed in Chapter 4 relate to the preoperative 2D path planning of needle motion, where 3D needle steering can be achieved through a proper controller, which stabilizes the needle to the plane of interest. In fact, 3D paths would allow more possibilities than planar paths [52]. Also, preoperative path-planning approaches are not suitable 
candidates for real-world applications. However, in this chapter, we focus on achieving autonomous needle steering within a simulated biological soft tissue and in a simulated real-world scenario through integrating the needle tip tracking system with intraoperative needle tip path replanning. Thanks to the low time-complexity of RRT planners, it is possible to perform intraoperative fast path re-planning, a necessary step for systems undergoing unpredicted changes, as it is the case with real-world applications.

Placing the needle tip accurately during needle motion inside a biological soft tissue is, at once, challenging and critical for exact robotic needle steering. In the context of closed-loop control of the needle tip, different image-guided control methods have been proposed. The authors in [65] and [94] used charge-coupled device (CCD) cameras to provide the needle tip position and orientation. However, the use of CCD cameras as an imaging modality is not clinically applicable given the invisibility of targets. Other various medical imaging modalities including ultrasound, CT, fluoroscopy, EMS, and MR imaging have been widely used in different needle steering systems. However, there are certain complications with many of these imaging techniques. The use of X-ray imaging such as $\mathrm{CT}$ or fluoroscopy exposes the patient to high doses of ionizing radiation [95]. Electromagnetic position tracking sensors [96] can be used for 3D needle tracking, but the presence of ferrous materials in the range of measurement can affect the accuracy of this method. MR imaging suffers from a low refresh rate and incompatibility with ferromagnetic materials [97]. Among all these imaging techniques, ultrasound imaging is preferable. This is because of its potential for real-time applications, compatibility with the needle material, safety in terms of no radiation exposure, and low cost. Regarding 3D US imaging, there is a compromise between the voxel resolution and acquisition time. Therefore, the US transducers used for real-time applications have limited resolution and the refresh rate of a 3D image is low [98]. To overcome this problem, Vrooijink et al. in [99] 
introduced a real-time 3D flexible needle tracking algorithm using 2D transverse US images. In order to consistently view the needle tip in the US images, the US probe is robotically repositioned to move in tandem with the needle tip. This is achieved by a compensator, where the needle insertion velocity is corrected by needle tip velocity to compensate for out-of-plane motions. Due to inherent noise, there is an aberration in the probe position which is compensated for using Kalman filter in a closed-loop configuration. They estimated the needle tip pose with the assumption that both needle torsional stiffness and buckling along the needle shaft are neglected.

Some of the introduced methods [59], [84], and [100] address intraoperative replanning only in static environments since these methods are based on information obtained preoperatively. The authors in [100] developed a planning approach based on an offline optimization algorithm, where their solution generates a lookup table that enables real-time image-guided control of the steerable needle in a static environment. However, when it comes to a real-world scenario, patient motion and physiological changes should be reckoned with in the planning approach. An alternative approach that works well in dynamic environments is the use of fast intraoperative planners that could update the planned trajectory online based on imaging feedback information. Our planner is based on this approach and can accurately and autonomously steer the bevel-tip needle during insertion inside a three-dimensional anatomy. Since our planner is not based on prebuilt roadmaps, it can react to perturbations seen in target motion, which could be approximately $7 \mathrm{~mm}$ throughout the procedure in a breast biopsy [101], [102], and an average and a maximum of $6.8 \mathrm{~mm}$ and $21.6 \mathrm{~mm}$, respectively, during prostate brachytherapy [103]. In addition to a dynamic workspace as a characteristic of a real-world needle insertion procedure, tissue variability is of high importance. A real living tissue is inhomogeneous and anisotropic. It is known that the use of duty-cycle approach in an automatic needle steering system calls for a 
good knowledge about the needle maximum curvature. However, for a real living tissue, this data is unknown or uncertain prior to a needle insertion procedure and more importantly varies during a procedure. Although an offline estimation of the needle maximum curvature may be sufficient for preoperative planning in tissue phantoms, for real tissue the needle maximum curvature has to be continuously updated through real-time needle tip imaging feedback. Hence, image-guided online needle curvature estimation is a key step in accurate autonomous needle steering within biological soft tissue.

The authors in [13] developed a robotic needle steering system for a 3D dynamic environment using the 2D ultrasound imaging feedback introduced in [99]. Moreira et al. in [12] integrated the system introduced in [13] with the online curvature estimation using the ultrasound imaging feedback (See Appendix B for details). However, the scope of both works was limited to a single-target path-planning problem for a steerable needle. In this chapter, we extend the work of [12] to the real-time path planning of steerable needles in environments with multiple targets in simulated realworld applications. According to [12], the overall robotic needle steering system is composed of different components: duty-cycled needle steering, online curvature estimation, ultrasound-based needle tracking, and a fast RRT-based multi-target path planning. A schematic diagram of the closed-loop system has been given in Fig. 5.1. As observed in Fig. 5.1, the needle tip pose is measured in real time and, subsequently, is fed into the online curvature estimation and multi-target path-planning subsystems to update the curvature estimation and the path planning algorithm, respectively. The needle curvature is estimated online, and, subsequently, the estimated needle maximum curvature is used to adaptively update the needle planned trajectory and duty-cycled steering. According to [99], the needle tracking frequency based on ultrasound imaging feedback is $25 \mathrm{~Hz}$. Also, both the motion planning and duty-cycled steering are executed continuously at a frequency of $1 \mathrm{~Hz}$. 


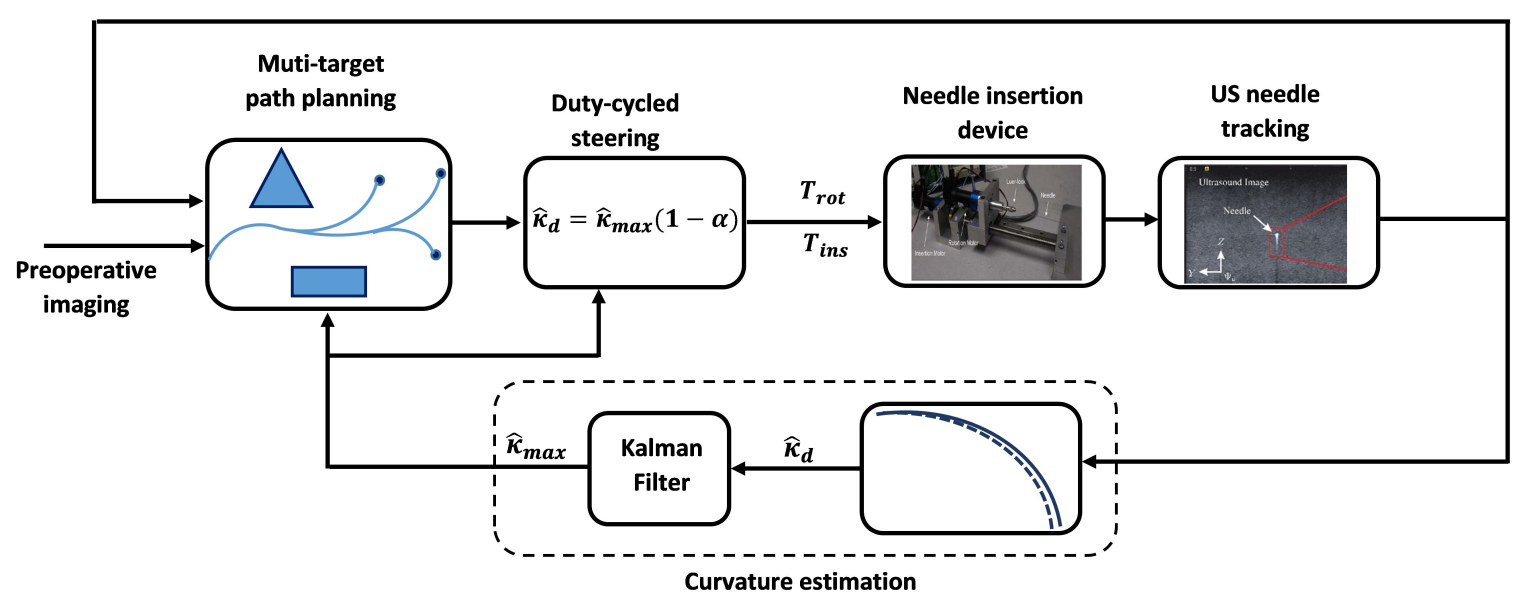

Figure 5.1: Closed-loop configuration of a robotic needle steering system. The needle insertion device robotically inserts and rotates the needle base during intervals of $T_{\text {ins }}$ and $T_{\text {spin }}$ defined by the duty-cycled steering algorithm. The desired needle tip trajectory is computed by the RRT-based multi-target path-planning algorithm. The needle path curvature and the maximum curvature are both defined by the estimation algorithm. The needle tip pose is estimated online through the real-time ultrasound needle tracking system, adopted from [12].

\subsubsection{Assumptions}

Before starting the design of the path-planning algorithm, a few assumptions have been made in our work. As stated earlier, our study aims for simulated real-world applications of flexible bevel-tip needle insertion within soft tissue. On the other hand, since our work is based on simulation results, to compensate for the lack of actual experimental measurements, we fabricate the necessary data to make our simulations as close to real-world scenarios as possible. In this regard, the following assumptions have been made in our work.

- We assume that we have access to medical images to precisely specify the geometry of the environment including obstacle definitions and the locations of targets in real time. As introduced in [99], using 2D Ultrasound images, 3D realtime needle tip tracking is feasible. In this regard, we fabricate the 3D needle 
tip pose measurements by manipulating the nominal values obtained through the model and some random values.

- Since the online estimation of the needle tip curvature is based on the measured data of the needle tip pose as introduced in [12] and shown in Fig. 5.1, we simulate the online estimation of the needle tip curvature $\hat{\kappa}_{d}$ by fabricating the respective data through manipulating the model curvature parameter with random values. The needle maximum curvature $\hat{\kappa}_{\max }$ is also obtained through $\hat{\kappa}_{d}$ and the model curvature parameter.

- As in the path-planning problem in Chapter 4, we assume a completely deterministic model for needle motion and do not explicitly consider uncertainty in needle motion due to tissue inhomogeneity and tissue deformation. For needle motion model, we assumed that the needle tip follows a circular arc with a constant radius of curvature in the direction of the bevel as introduced by Webster et al. in [14]. It is assumed the tissue is stiff relative to the needle and the needle is torsionally stiff. Consequently, the needle motion is completely characterized by its tip motion and controlled by two degrees of freedom, namely the needle insertion velocity $\boldsymbol{v}$ and the needle shaft rotational velocity $\boldsymbol{\omega}$ both applied at the needle base.

As shown in Section 5.5, various scenarios have been tried in the simulations, with close attention to the practicality of the simulations. In this regard, different simulations to replicate homogeneous and inhomogeneous real tissue with a wide range of tissue property variability are presented. It bears noting that despite all the best effort to simulate the real-world application, there are still specific issues regarding a real-world scenario that are not accounted for in our work. For instance, the needle tip tracking system may not be able to differentiate the needle tip from the internal structures of the soft tissue, which could result in errors in estimating the 
needle tip position. Therefore, to address any potential issues that could be expected in the practical deployment of any designated approach, in-vivo tests have to be done before transferring any proposed methodology into operating rooms.

\subsection{Single-Target Motion Planning}

To begin with, we introduce our planner for a single target. In a classic RRT algorithm [57], instead of discretizing the configuration space, the tree is expanded incrementally by sampling a random configuration $q_{\text {rand }}$ from the free continuous state space based on a predefined sampling distribution. Subsequently, the algorithm finds the node $q_{\text {near }}$, which is the closest node to the sampled configuration $q_{\text {rand }}$, based on a specific distance metric $\rho(q)$. Then, the algorithm tries to expand the tree toward $q_{\text {rand }}$, based on the best control input $u$ that navigates the configuration $q_{\text {near }}$ toward $q_{\text {rand }}$. The resulting configuration $q_{\text {new }}$ is added to the tree as the new node. However, for nonholonomic systems like the steerable needle, finding the best control input to reach a sampled state requires solving a difficult two-point boundary value problem of connecting two states in $\mathrm{SE}(3)$. Some classic RRT-based methods [58] and [74] avoid solving this difficult problem by applying a deterministic or uniform random sampling of the control inputs $u=(v, \omega)$ in finding the new node $q_{\text {new }}$ that is closest to $q_{\text {rand }}$. Since these methods are based on a constant-curvature kinematic model, which is a very restrictive constraint on needle motion inside soft tissue, needle motion toward the sampled state requires a great number of control inputs, resulting in high computational costs of the algorithm. The authors in [15] did without a random sampling of control inputs to find the best control input to expand the tree. They encoded segments of a needle trajectory based on geometric quantities instead of control inputs. The needle trajectory is composed of a piece-wise circular arc segments $C_{i}$, each parameterized as a triplet $\left(l_{i}, r_{i}, \phi_{i}\right)$. This geometric representation of needle 
path could alleviate the computational cost significantly. Later, control inputs can be extracted from the computed trajectory, more about this below. In this regard, a random point $P_{\text {rand }}$ is sampled instead of a random configuration from the state space. Like [15], [18], and [12], we assume variable-curvature needle motion inside soft tissue and use the RRT planning algorithm along with a reachability-guided sampling heuristic to make the method efficient for the nonholonomic kinematic model of the needle motion. In this regard, according to Fig. 3.4 in Chapter 3, the reachability region of the needle tip at its local coordinate frame consists of all the points $p_{\text {rand }}:\left\{p_{x}, p_{y}, p_{z}\right\} \in R^{3}$ that can be reached by the needle tip. Mathematically, the reachability region is inside the volume of a region expressed by (5.1), where $r_{0}$ is the needle natural curvature.

$$
p_{z} \geq \sqrt{2 r_{0} \sqrt{p_{x}^{2}+p_{y}^{2}}-\left(p_{x}^{2}+p_{y}^{2}\right)}
$$

By adapting the traditional sampling [58] to the reachability-guided sampling [63], the planning benefits significantly through an efficient expansion of the rapidly exploring search tree. While checking the reachability of the sampled point, limiting the needle path curvature to a constant value as expressed in [14] reduces the probability of accessing a randomly sampled point in the configuration space, particularly through minimum-length trajectories, thus reducing the planner's efficiency as the number of attempts made to reach that sampled point increases. Consequently, as in the discussion in Chapter 4, we adopt variable-curvature needle motion kinematics in our planning algorithm.

The needle tip configuration is expressed by the needle tip pose as $X=\left[\begin{array}{ll}R & P \\ 0 & 1\end{array}\right]$, where $R \in S O(3)$ and $P \in \mathbb{R}^{3}$ are the needle tip orientation and position expressed in inertial frame, respectively. For a sampled point $P_{B}=P_{\text {rand }} \in R^{3}$ and a given configuration $X_{A}=X_{\text {near }}=\left[\begin{array}{cc}R_{\text {near }} & P_{\text {near }} \\ 0 & 1\end{array}\right]$, there exists a circular arc segment $C_{i}$, 
characterized by $(l, r, \phi)$, where $l$ is the arc length, $\phi$ is the change of the bevel tip orientation (which is equal to the change of the orientation of the needle tip coordinate frame $X_{\text {near }}$ around the $z_{\text {near }}$-axis), and $r$ is the radius of the circular $\operatorname{arc} C_{i}$. This is shown in Fig. 5.2.

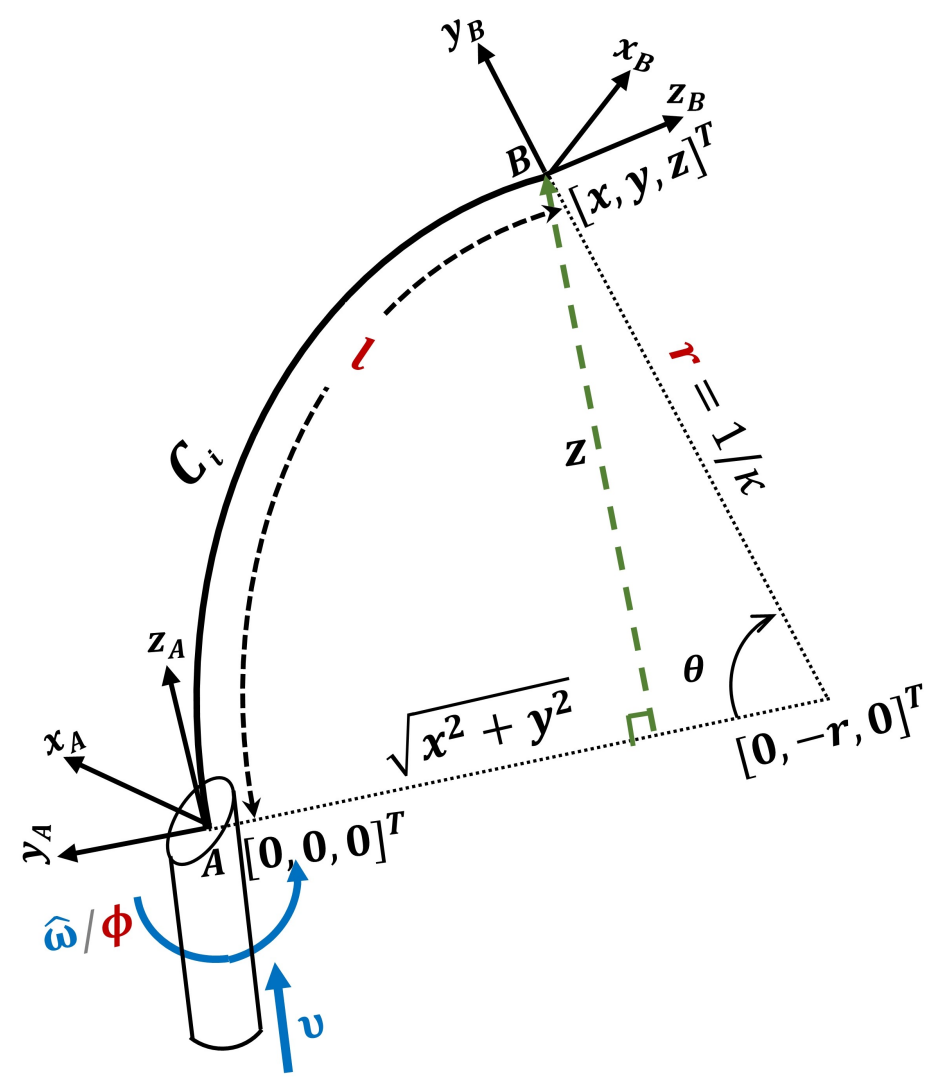

Figure 5.2: Geometric representation of a piece-wise circular arc segment $C_{i}$ which is characterized by $(l, r, \phi)$ where $\phi$ is the change of bevel direction, $l$ and $r$ are the arc length and radius, respectively, regenerated from [18].

To make the transition from $X_{A}$ to $P_{B}$ possible, first, the direction of the bevel tip has to be reoriented by $\phi$ radians to align the needle motion on the plane containing $P_{A}$ and $P_{B}$. This is done by rotating needle shaft $z_{A}$ by $\phi$ radians. Afterward, based on the DC approach, by applying $v$ and $\omega$ to the reoriented needle tip according to (4.3) in Chapter 4, the needle tip moves along $C_{i}$ to reach the sampled position $P_{B}$. The parameters of the respective circular arc segment $C_{i}$ shown in Fig. 5.2 can be 
uniquely obtained according to (5.2)-(5.4), where $[x, y, z]^{T}$ is the relative coordinate of $P_{B}=P_{\text {rand }}$ expressed in the local coordinate frame of $X_{A}=X_{\text {near }}$ and can be written as $[x, y, z]^{T}=R_{\text {near }}^{T}\left(P_{\text {rand }}-P_{\text {near }}\right)$.

$$
\begin{aligned}
& r=\frac{x^{2}+y^{2}+z^{2}}{2 \sqrt{x^{2}+y^{2}}} \\
& l=r \theta=r \cdot \arctan 2\left(z, r-\sqrt{x^{2}+y^{2}}\right) \\
& \phi=\arctan 2(x,-y)
\end{aligned}
$$

Due to the nonholonomic constraint of the needle motion, $P_{B}$ has to be inside the reachability region of the needle tip at $X_{A}$. Mathematically, the parameter $r$ in (5.2) must satisfy $r \geq 1 / \kappa_{0}$. Also, to prevent needle buckling, parameter $\theta$ obtained in (5.3) has to be limited to $90^{\circ}$.

The Homogeneous matrix $G_{i}$, corresponding to circular arc segment $C_{i}$, which represents the needle tip configuration of $X_{\text {new }}$ in the local coordinate frame $X_{\text {near }}$ is as follows.

$$
G_{i}=\left[\begin{array}{cccc}
\cos \phi & -\sin \phi \cos \theta & \sin \phi \sin \theta & r \sin \phi(1-\cos \theta) \\
\sin \phi & \cos \phi \cos \theta & -\cos \phi \sin \theta & -r \cos \phi(1-\cos \theta) \\
0 & \sin \theta & \cos \theta & r \sin \theta \\
0 & 0 & 0 & 1
\end{array}\right]
$$

According to (5.5), where $G_{i}$ is defined for each circular arc segment, the needle tip configuration at $X_{\text {new }}$ in the world reference frame can be obtained through (5.6), where $N$ is the number of circular arc segments traversed by the needle tip from the initial configuration $X_{0}$ toward $X_{\text {new }}$. 


$$
G_{w n}=\prod_{i=1}^{N} G_{i}
$$

The initial orientation of the needle tip $R_{0}$ plays an important role in the quality of the tree branching toward $P_{\text {goal }}$. The nominal value for $R_{0}$ is calculated as the required change of the needle tip orientation in connecting $X_{0}=\left[\begin{array}{cc}I_{3 \times 3} & P_{0} \\ 0 & 1\end{array}\right]$ to $P_{\text {goal }}$ through a single arc, as shown in Fig. 5.2, which can be obtained through three consecutive and independent elementary rotations. Subsequently, each time the planner is iterated, we apply a normal distribution to sample each of these rotation angles. Moreover, we limit the dimensions of the sampled point to be almost around the cube connecting $P_{0}$ to the target. In case one side of the cube is much smaller that the other two, an extra margin is added accordingly. The reason is obvious: since the needle path curvature is limited, sampling points very far from this area require the needle to return toward the target which is almost impossible in clinical metrics.

From the initial state $X_{0}$ the planner incrementally builds $\mathfrak{T}$ toward $P_{\text {goal }}$ by sampling an obstacle-free position $P_{\text {rand }}$. Then, the planner checks for feasible connectivity of $P_{\text {rand }}$ with the current vertices in the $\mathfrak{T}$ to construct the reachable set $Q_{\text {reach }}$. Afterward, the planner finds the closest state $X_{\text {near }}$ among $Q_{\text {reach }}$ based on a specific distance metric $\rho()$, expressed in (5.7), to expand the $\mathfrak{T}$ toward the potential new state $X_{\text {new }}$, which has the same position as of $P_{\text {rand }}$.

$$
\rho\left(X_{\text {near }}, P_{\text {rand }}\right)= \begin{cases}l(\equiv r \theta), & \text { if } r \geq 1 / \kappa_{0} \wedge \theta \geq 0 \\ \infty, & \text { otherwise }\end{cases}
$$

As expressed in (5.7), the arc length is considered as the distance metric if $P_{\text {rand }}$ is inside the reachability region of $X_{\text {near }}$; otherwise, it is considered infinity. Finally, if this connection is obstacle-free, $X_{n e w}$ and the corresponding arc segment connecting 
$X_{\text {near }}$ to $X_{\text {new }}$ are added to the $\mathfrak{T}$ as the new vertex and edge, respectively. This process goes on until either the $\mathfrak{T}$ is connected to $P_{\text {goal }}$ or the available time is exceeded. Once the needle has reached the target region, the plan is extracted. Thanks to fast computation of RRT planner, the planner is iterated many times in order to have a set of feasible motion plans. Based on a clinician's specified cost function, the best plan is extracted. The RRT planning algorithm for a single target in a 3D space is expressed in $\mathrm{ST}-\mathrm{RRT}_{3 \mathrm{D}}$, which is similar to $\mathrm{ST}-\mathrm{RRT}_{2 \mathrm{D}}$.

${\mathbf{S T}-\mathbf{R R T}_{\mathbf{3 D}} \text { 3D Single-Target RRT Planning Algorithm }}$

1: $\mathfrak{T} \leftarrow$ Initialize_path $\left(P_{0}, P_{\text {goal }}, \tau\right)$

2: while $\left(\mathfrak{T} \cap P_{\text {goal }}=\emptyset \& t \leq \tau\right)$

3: $\quad P_{\text {rand }} \leftarrow$ biased-obstacle-free_random_position $\left(\Re^{3}\right)$

4: $\quad Q_{\text {reach }} \leftarrow$ reachable_set $\left(P_{\text {rand }}, \mathfrak{T}\right)$

5: $\quad X_{\text {near }} \leftarrow$ closest_neighbor $\left(Q_{\text {reach }}, P_{\text {rand }}, \rho()\right)$

6: $\quad(l, r, \phi) \leftarrow$ circular_arc $\left(X_{\text {near }}, P_{\text {rand }}\right)$

7: $\quad u \leftarrow$ control_inputs $((l, r, \phi), \delta)$

8: $\quad X_{\text {new }} \leftarrow$ new_state $\left(X_{\text {near }}, u, \delta\right)$

9: $\quad$ if collision_free $\left(X_{\text {near }}, u, \delta, X_{\text {new }}\right)$

10: $\quad \mathfrak{T} \leftarrow$ add_vertex $\left(X_{\text {new }}\right)$

11: $\quad \mathfrak{T} \leftarrow$ add_edge $\left(X_{\text {near }}, u, \delta, X_{\text {new }}\right)$

12: $\quad$ end

13: $\quad$ if $P_{\text {new }} \in P_{\text {goal }}$

14: $\quad \mathfrak{P} \leftarrow$ extract plan $(\mathfrak{T})$

15: end

16: update $t$

17: end

18: return $\mathfrak{P}$ 


\subsection{RRT-Based Intraoperative Motion Plan}

Our motion planner is fast enough to compensate for different sources of uncertainty and perturbations in needle motion, target and obstacle motion as they occur. Therefore, the control system can autonomously drive the needle toward moving targets, while at the same time avoiding any possibly moving obstacles. Given the preoperative medical images, the initial locations of targets and obstacles are known and the needle's initial insertion location can be determined. We adapt the RRT-based motion planner with some heuristics introduced in Section 5.2 to make it a proper candidate for real-time control of needle steering. The simulation results in Section 5.5 verify that our system can autonomously reach multiple targets with a noticeably higher targeting accuracy than the current clinical practice, while it guarantees obstacle avoidance, too.

Our planning approach is based on a nonholonomic deterministic model for the needle motion. To compensate for different sources of uncertainty arising from uncertain needle-tissue interaction, tissue inhomogeneity, and tissue deformation, which result in target and obstacle motion, the planning is repeated at intervals of $\Delta$ s. Only the initial plan is executed for $2 \Delta \mathrm{s}$ to obtain the necessary measured data available for the next planning interval. During each interval $\Delta_{k}$, the needle steering system executes the plan computed during the previous interval $\Delta_{k-1}$, while at the same time the planner computes the plan for the next interval $\Delta_{k+1}$. During each $\Delta_{k}$, the planning is in process based on the following inputs given at the beginning of $\Delta_{k}$.

- The estimated needle tip pose at the end of $\Delta_{k}\left(X_{n e s t}\right)$ : this estimation is done based on the measured needle tip pose at the beginning of $\Delta_{k}\left(X_{0 \text { mes }}\right)$ and using the needle path curvature obtained through the planner from the previous plan.

- Estimated needle natural curvature during $\Delta_{k-1}$ : this estimation is obtained 
through the measured needle path curvature during $\Delta_{k-1}\left(\kappa_{m e s}\right)$ and the respective applied duty-cycle factor.

- The updated measured data of targets and obstacles locations at the beginning of $\Delta_{k}$.

To simulate a real-world scenario, we had to fabricate the needle actual trajectory. It is done by adding random values to the model parameters. Since it is assumed the needle is inserted by a fixed length at each $\Delta, \kappa_{\text {model }}$ and $\phi_{\text {model }}$ parameters of the best plan are randomly manipulated in order to simulate an in-homogeneous tissue. In section 5.5, simulations show the clinical diversity of the fabricated data in simulating an in-homogeneous living tissue. Finally, the re-planning process continues till the z-coordinate of the measured needle tip position and the estimated target location are almost equal.

\subsection{Intraoperative Multi-Target RRT-based Path- Planning Algorithm for Flexible Bevel-Tip Needle}

Through the intraoperative multi-target RRT-based path-planning algorithm, it is possible to immediately generate new motion plans as any change in the system is happening. The objective is to find a sequence of discrete control actions (motion plans) that can automatically lead the steerable bevel-tip needle navigation inside 3D biological soft tissue in real time to reach multiple targets, while simultaneously avoiding collision with obstacles in a real-world application with as little tissue trauma as possible. To address the issue of uncertainty due to tissue inhomogeneity and unexpected perturbations arising from the obstacle/target motion, unlike [59], [60], [67], 
which explicitly consider uncertainty in needle motion, we use the deterministic unicycle model [14] in this work. Consequently, the planning is repeated intraoperatively at intervals of $\Delta \mathrm{s}$ based on the most recent feedback data obtained from the needle tip pose, needle path curvature, targets and obstacles positions. Once the first target has been accessed, the needle is retracted partially, re-oriented, and reinserted towards the next target. This process goes on till all the targets are reached. Without having to completely retract the needle for each target, we can considerably decrease the associated tissue trauma this way.

Our Multi-Target RRT Planning Algorithm is outlined in MT-RRT $3 \mathrm{D}$. The input to the algorithm is the same as the one to $\mathrm{ST}-\mathrm{RRT}_{3 \mathrm{D}}$; however, instead of a single target we have a set of targets defined as $S_{T}$. 


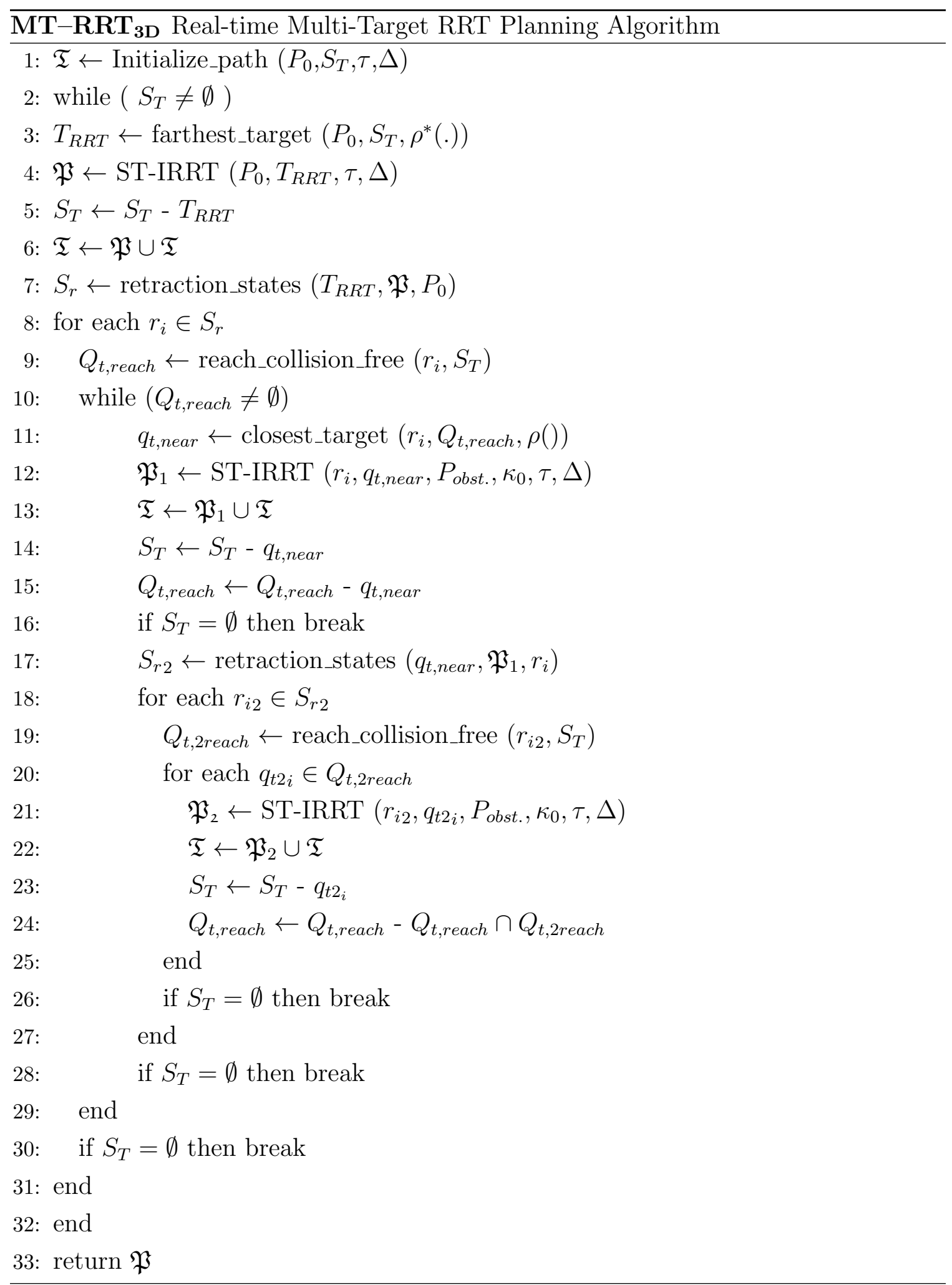


As described in $\mathrm{MT}-\mathrm{RRT}_{3 \mathrm{D}}$, to begin with, based on the Euclidean distance metric $\rho^{*}($.$) , the planner selects the farthest target as the first target T_{R R T}$ to iterate the intraoperative RRT planning (ST-IRRT). $\mathfrak{P}$ is the output generated through STIRRT which will be used during the needle retraction phase to access the other targets. Each time the needle visits a target, we remove that target from $S_{T}$ for the rest of the algorithm. Therefore, during the algorithm, $S_{T}$ represents the set of unvisited targets. After accessing the first target, the needle retracts along $\mathfrak{P}$ incrementally till the root node. In doing so, the planner calculates a certain number of states starting with $T_{R R T}$ and finishing with $P_{0}$, called retraction states $r_{i} \in S_{r}$, which are equally spaced per circular arc segment of $\Delta \mathrm{s}$. At each retraction state $r_{i} \in S E(3)$, the planner checks the collision-free reachability of all the unvisited targets $S_{T}$ through function reach_collision_free in $\mathrm{MT}-\mathrm{RRT}_{3 \mathrm{D}}$. The set of collision-free reachable targets from retraction state $r_{i}$ is denoted by $Q_{t, \text { reach }}$. The planner first checks for the closest target $q_{t, \text { near }}$ in $Q_{t, \text { reach }}$, where the arc length is chosen as the distance metric $\rho()$. Then, the planner iterates the intraoperative ST-IRRT planning starting from $r_{i}$ to reach $q_{t, \text { near }}$. The resultant path is $\mathfrak{P}_{1}$. In a similar vein, the needle retracts along $\mathfrak{P}_{1}$ incrementally through the set of retraction states $r_{i 2} \in S_{r_{2}}$ starting from $q_{t, \text { near }}$ till $r_{i}$. This extra level of examining the collision-free reachability of the unvisited targets is done to improve the planning efficiency. During retraction on $\mathfrak{P}_{1}$, for any $r_{i 2}$ that the collision-free reachable set $Q_{t, 2 \text { reach }} \neq \emptyset$, the planner iterates ST-IRRT to reach the respective target. The planning process terminates whenever $S_{T}=\emptyset$.

\subsection{Simulations and Discussion}

We implemented our motion planner in Matlab R2018a and tested it on a $3.6 \mathrm{GHz}$ Core i7 CPU. We set $\Delta=\tau=1 s$, the initial needle natural curvature to be $\kappa_{0}=$ $0.2 \mathrm{~cm}^{-1}$ and defined the workspace dimensions and needle entry location $P_{0}$ as 
$5 \times 5 \times 10 \mathrm{~cm}^{3}$ and $[0,0,0]^{T}$, respectively. Fig. 5.3 shows the tree-like planned trajectory by our new multi-target planner for the needle to reach randomly chosen targets $T_{1}$ through $T_{7}$. It is clear that by using our new multi-target RRT-based planning Algprithm in $\mathrm{MT}-\mathrm{RRT}_{3 \mathrm{D}}$, the costs associated with the needle insertion procedure in Fig. 5.3 decrease substantially and, as a result, there is less tissue damage during the procedure.

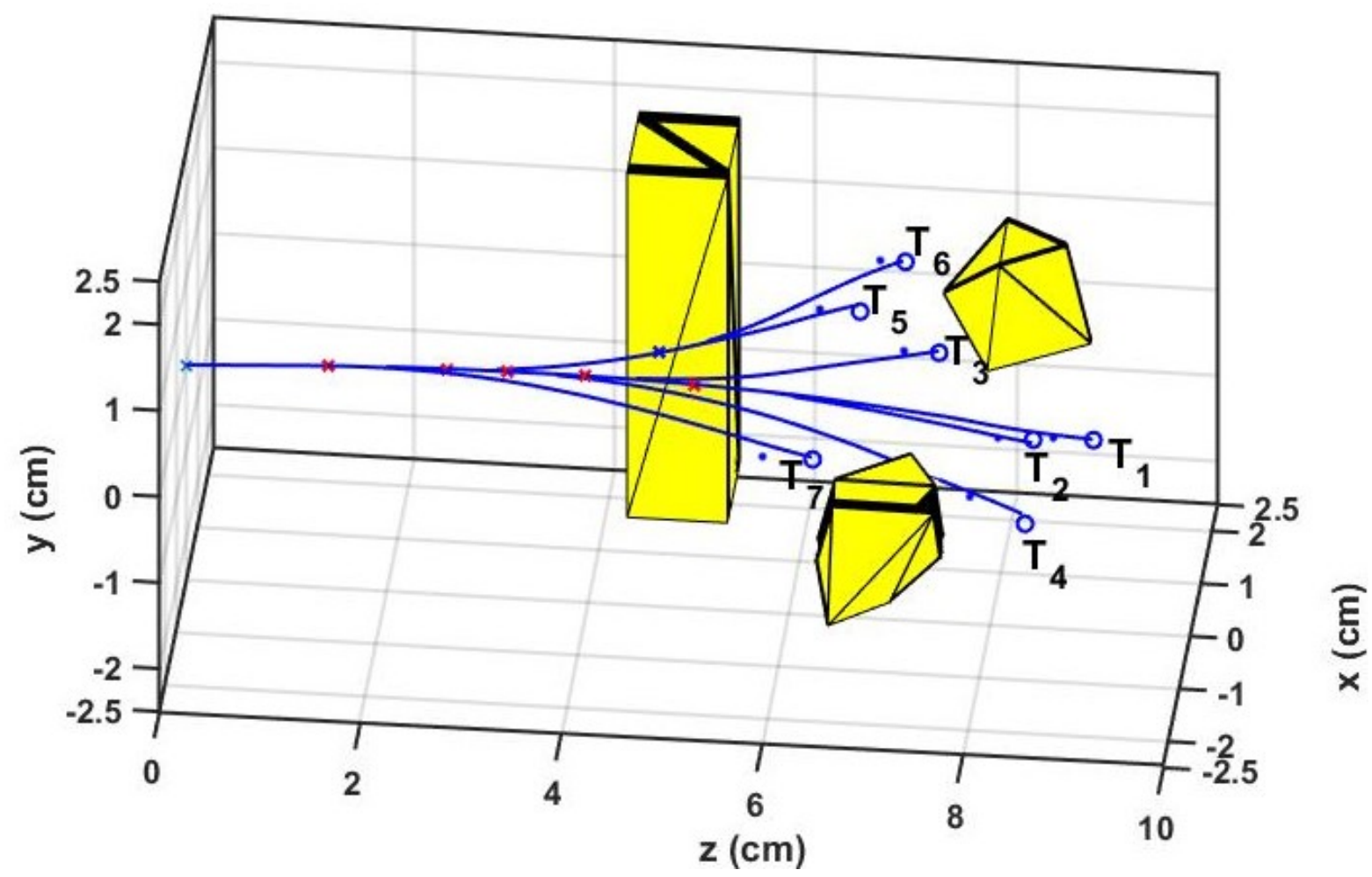

Figure 5.3: Novel multi-target RRT-based motion planning for the steerable needle inside in-homogeneous tissue including moving targets and obstacles. The initial and the final locations of the targets are shown in blue dots and circles, respectively. Target indices show the order in which each target is reached. Moreover, the retraction states $r_{i}$ and $r_{i 2}$ in MT- $\mathrm{RRT}_{3 \mathrm{D}}$ are mentioned in red and blue $\times$, respectively. The maximum and minimum targeting error is $1.023 \mathrm{~mm}$, $0.388 \mathrm{~mm}$ respectively.

Variation of the needle natural curvature during the path for each target in Fig. 5.3 is shown in Fig. 5.4. The results confirms the inhomogeneity of the tissue including different layers and structures. 


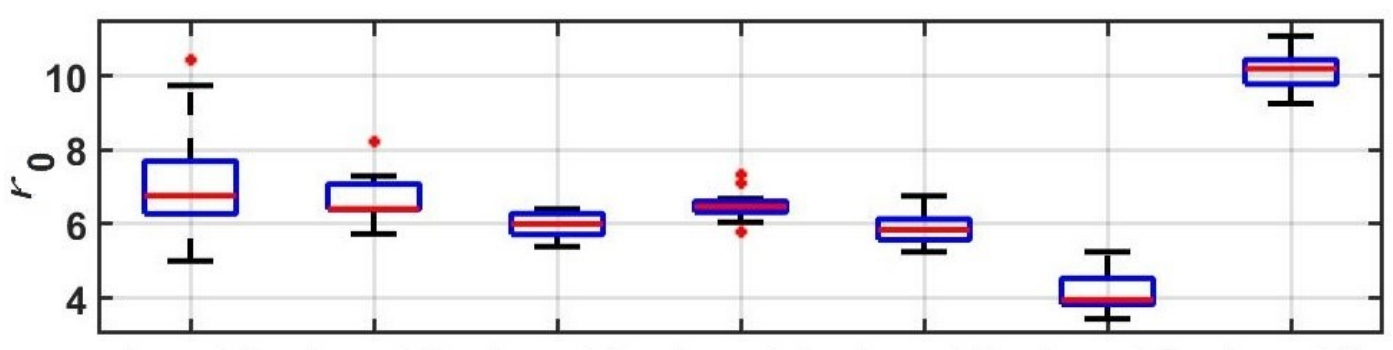

target 1 target 2 target 3 target $4 \quad$ target 5 target $6 \quad$ target 7

Figure 5.4: In-homogeneous properties of the tissue used in Fig. 5.3.

Fig. 5.5 provides a quantitative analysis of our new real-time multi-target RRT-based planner for in-homogeneous tissue to reach multiple randomly-chosen targets. Both target and obstacle motion is considered in the simulations.
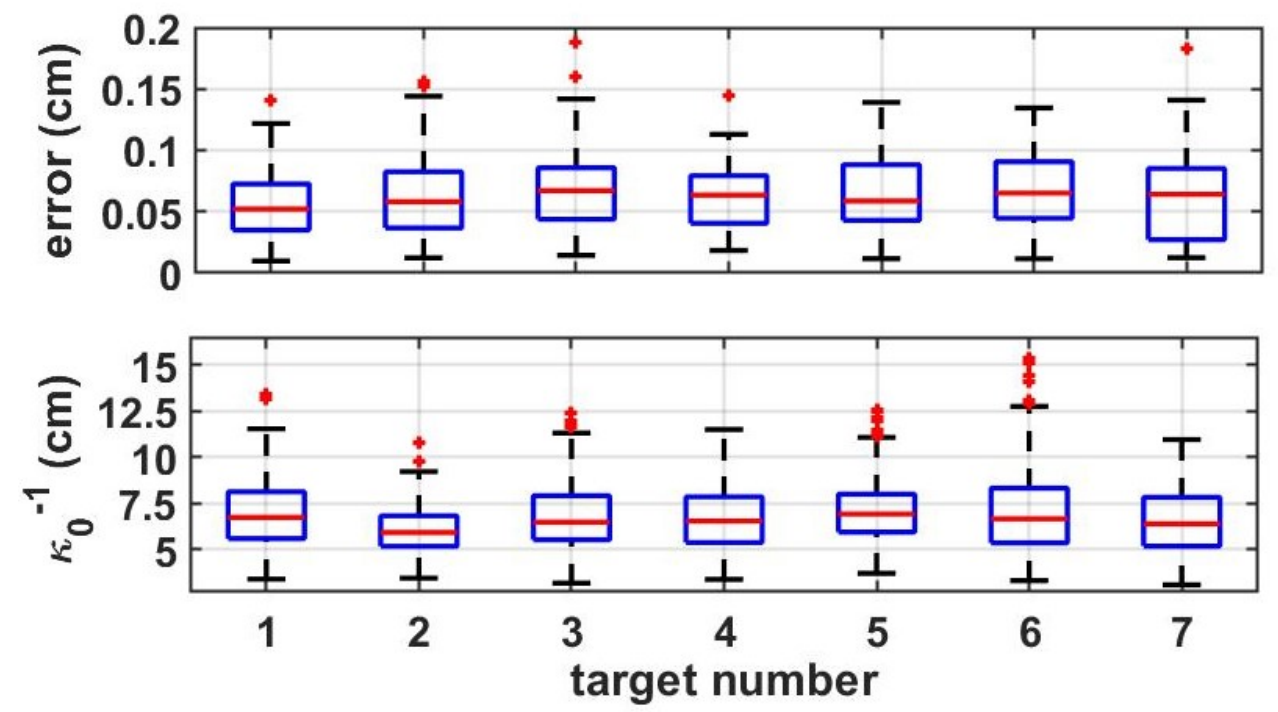

Figure 5.5: Evaluation of our new path planner for steerable needle motion inside in-homogeneous tissue in a simulated real-world application. a) targeting error per target and b) variation of the needle natural curvature during the path for each target. All results correspond to 50 simulations.

The results for the needle natural curvature variation and the targeting error, first and foremost, reveals that the planning is efficient under tissues with different structures and including membranes as it is the case in real-world applications. The error data 
also shows the efficiency of the planner in reaching each target accurately.

Fig. 5.6 provides a quantitative comparison of our new planning approach with the traditional approach which is based on the sequential insertion and retraction of the needle for each separate target. Without loss of generality, we limit the simulations to homogeneous tissue and just focus on the target motion with the hope of providing a more consistent environment for both scenarios. As shown, the cost associated with needle insertion drops by an average of $36 \%$ through our new planner since the need to complete retraction from and insertion to each separate target is obviated.
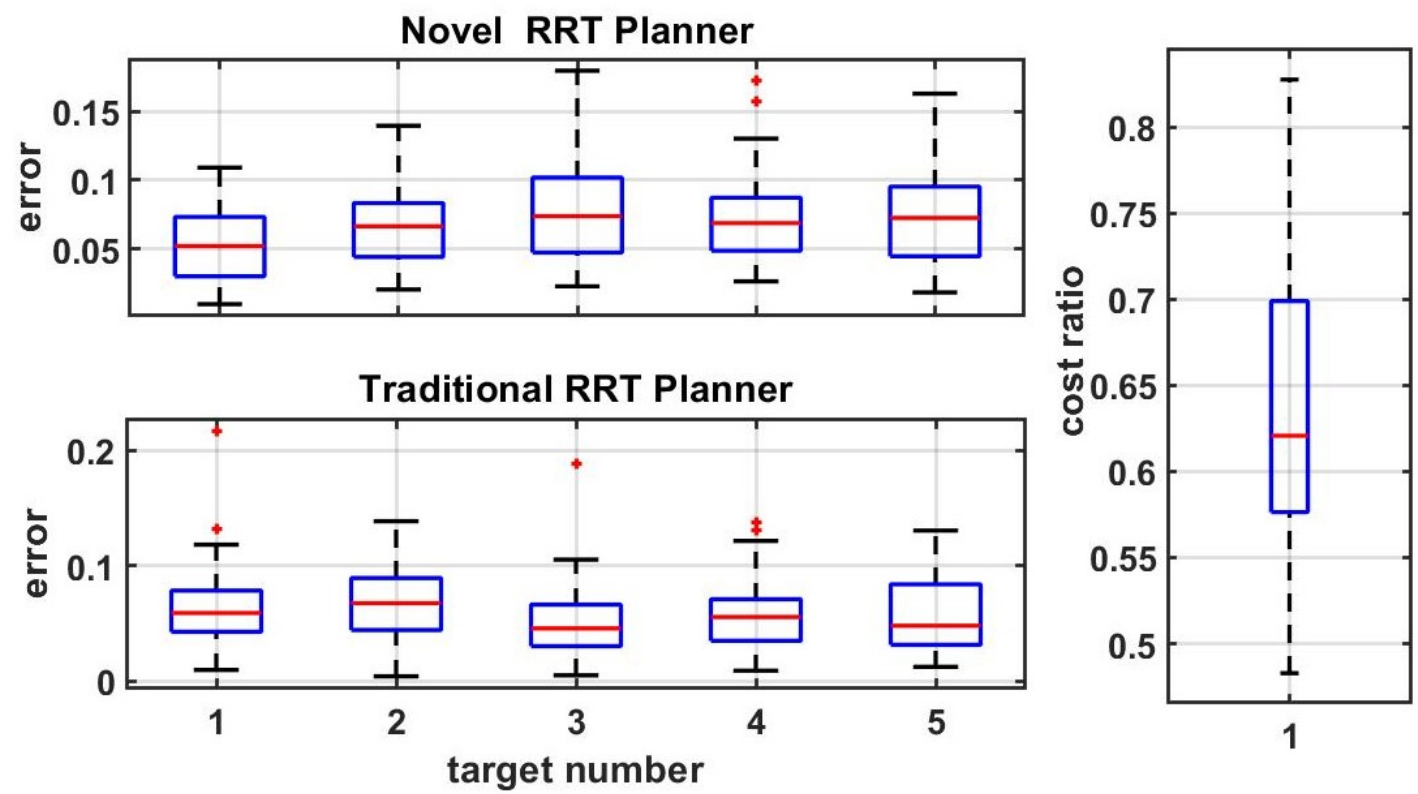

Figure 5.6: The performance of our new intraoperative RRT-based planner in a homogeneous tissue to reach five randomly chosen moving targets. a) The two left-sided plots show the targeting error of both our novel planner and the traditional one. b) The right-sided plot shows the cost ratio of our new planner vis-a-vis the traditional planner. All results correspond to 50 simulations. 


\subsection{Summary}

Thanks to the low time complexity of RRT planners, it is possible to perform intraoperative fast path re-planning, a necessary step for systems undergoing unpredicted changes, as it is the case with real-world applications. During each planning interval, the planner is iterated many times in order to have a set of feasible motion plans. Based on a clinician's specified criteria, the best plan is extracted as a high quality solution. To the best of the our knowledge, intraoperative path planning of steerable needle inside soft tissue to reach multiple targets in real-world application has never been presented anywhere in the literature before. Another notable feature of our planning algorithm is its practicality in terms of clinical metrics by focusing on a more clinically relevant choice of system parameters. Despite global optimizationbased methods with an exponential time complexity to the number of targets and system complexity, our planner performs well independently of these factors. More importantly, as the need for individual insertion and complete retraction of the needle for each target is obviated, the total tissue damage incurred during a needle insertion procedure decreases significantly. 


\section{Chapter 6}

\section{Conclusions}

This chapter presents conclusions of our study and suggests some possible future research on multiple needle steering.

\subsection{Thesis Conclusion}

- A new preoperative planar single-target planner with an improved performance is proposed by adding the heuristics to the RRT planning algorithm.

- A fast preoperative planar multi-target planner with improved performance is proposed. The planner can reach as many targets as required by the clinician very fast.

- A real-time intraoperative 3D multi-target planner for real-world applications is proposed. Autonomous steering of the flexible bevel tip needle is achieved within a biological soft tissue through integrating the 3D needle tip tracking system with intraoperative needle tip path replanning. Thanks to the low timecomplexity of RRT planners, it is possible to perform intraoperative fast path re-planning at intervals of 1 sec. Consequently, the planner can accurately react to patient variability or any unpredicted changes during needle motion, which 
is typical of needle steering within real physiological soft tissue.

- The 3D intraoperative planner can account for target and obstacle motion due to tissue deformations, patient motion, or physiological changes.

- The 3D intraoperative planner can compensate for different sources of uncertainty arising during needle motion including tissue variability, which is a characteristic of inhomogeneous real physiological soft tissue.

- Despite the global optimization methods, the proposed RRT-based planners perform well regardless of the number of targets and system complexity.

- Simulation results verify the improved performance of the proposed multi-target RRT-based planning algorithms vis-a-vis traditional planning methods. Since the need for individual insertion and complete retraction for each target is obviated, the total tissue trauma decreases considerably.

- All the proposed planners can navigate the needle in environments with obstacles and narrow passages.

- More importantly, the planners are based on a correct choice of system parameters including the needle natural curvature, workspace dimension, target region, and needle initial entry location. The clinical-relevance feature of the algorithm, which plays an important role in the motion planning performance, has been reckoned with in our work.

\subsection{Possible Directions for Future Research}

- For our future work, we aim to verify the efficiency of the proposed 3D real-time multi-target algorithm by doing experiments on real tissue. In this regard, we would like to update the algorithm accordingly. In particular, the relationship 
between the duty-cycle factor and the needle natural curvature, which is not always linear and mainly depends on the mutual characteristics of needle-tissue interaction with a general form of $h=\alpha(k)$, will be updated for real tissue experiments.

- We aim to run the code in a low-level programming language rather than in MATLAB, so that we can add more features to the motion planning algorithm, while at the same time we can keep the time complexity low enough for real-time applications. For instance, the needle initial entry location could be optimized over a permissible needle entry region.

- To provide a higher level of accuracy for a real-world application, we aim to add more realistic models of the needle's kinematic behavior. For example, needle torsional behavior could be included. It should be noted that adding any details to the needle motion behavior should not be at the expense of the time efficiency of the path-planning algorithm.

- For our future study, we are planning to examine our real-time path-planning algorithm for different clinical applications through customizing the original design of the asymmetric bevel-tip needle to offer enhanced procedure efficiency of the respective application.

- It is known that bevel-tip needle steering in soft tissue is obtained through tissue reaction forces. This results in inferior steerability during needle transition inside tissue structures with a lower Young modulus as fluid-filled cavities. This limitation is overcome by curved concentric tubes where interaction forces are independent of tissue stiffness. As a result, this can publicize their application into various medical procedures. In [104], a different means of needle steering based on curved concentric tubes is introduced and the details of both direct 
and inverse kinematics of these tubes were elaborated in quest of control and path planning. For future studies, we will extend our planning method to active steering of needles during tissue mediums with different stiffness properties. 


\section{Chapter 7}

\section{Breadth-First Search (BFS) Algorithm [1]}

A graph is denoted by $G=(V, E)$, where $V$ represents the set of vertices and $\mathrm{E}$ represents the set of edges, i.e. vertex pairs $(u, v)$. For each vertex $u \in V$, the adjacency of $u$ denoted by $\operatorname{Adj}[u]$ stores $u^{\prime} s$ neighbors, i. e. $\{v \in V \mid(u, v) \in E\}$.

A Breadth-First Search algorithm explores a graph level by level from the source vertex $s$ as follows.

- level $0=s$

- level $i=$ vertices reachable by path of $i$ edges but not fewer

- build level $i>0$ from level $i-1$ by trying all outgoing edges, but ignoring vertices from previous levels

In the following, an illustration of the Breadth-First Search algorithm and the Breadth-First Search algorithm are presented in Fig. 7.1 and Alg. BFS(), respectively. 

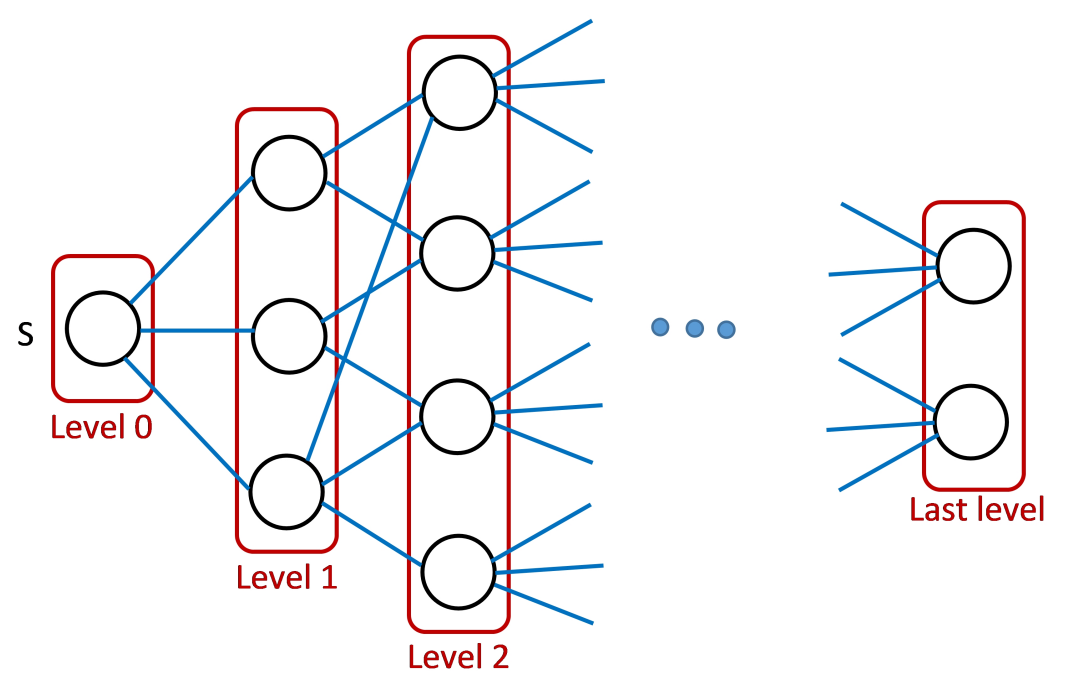

Figure 7.1: Demonstration of Breadth-First Search, regenerated from [1].

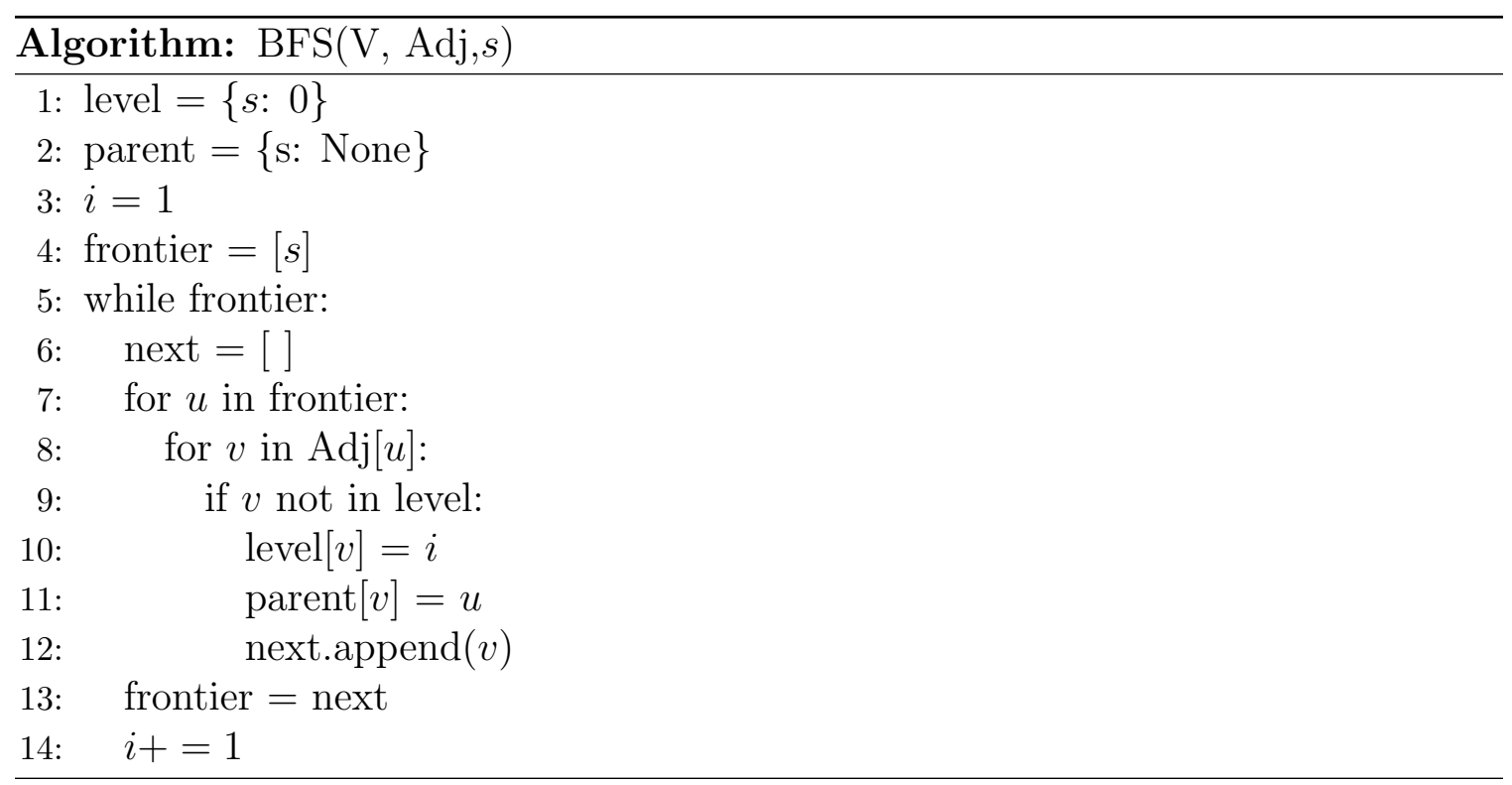




\section{Chapter 8}

\section{Curvature Estimation and Online}

\section{Adaptation}

The authors in [12] presented a method to adaptively adapt the relationship between desired needle path curvature $\kappa_{d}$ and duty-cycle factor $\alpha$. As shown in Fig. 5.1, the estimation process consists of two levels. First, based on the measured needle tip pose obtained through an ultrasound tracker, an online curvature estimation is performed, followed by a needle's maximum curvature adaptation. They define the needle trajectory as a series of circular arc segments each with constant curvature. These segments are called sub-trajectories. Each sub-trajectory is defined by one duty-cycle period, which is 1 second. Since the needle tracking frequency is $25 \mathrm{~Hz}$, during each duty-cycle period and equivalently for each sub-trajectory, $N=25$ needle tip pose measurements are recorded. To estimate the needle radius of curvature, a least squares (LS) algorithm is used for each sub-trajectory. In this regard, using the principle component analysis (PCA), the plane of the needle trajectory is extracted

from the measured data. As a result, the 3D needle curvature estimation problem is reduced to a planar LS problem. As shown in the algorithm below, the projected $P_{2 D}$ data represents the planar motion of the needle tip. An LS algorithm is used to best fit a circle to the $P_{2 D}$ data for each sub-trajectory, which is denoted by $\hat{\kappa_{d}}$. 


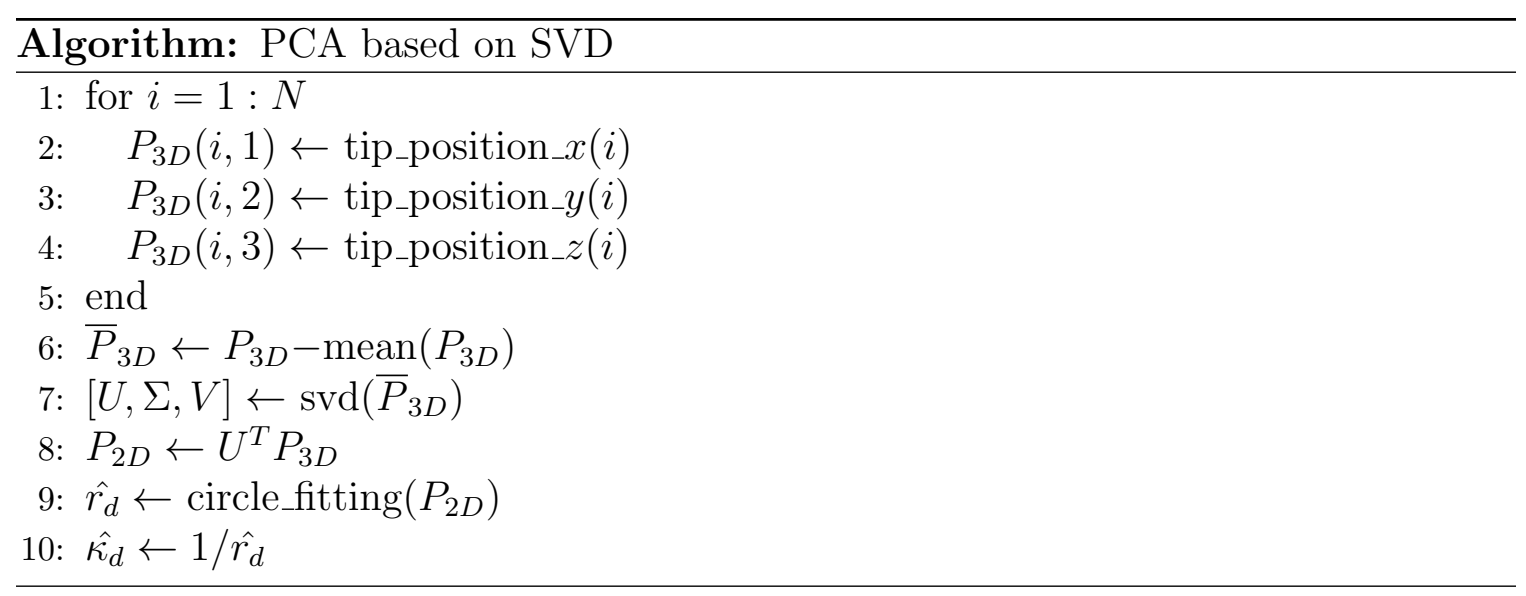

The PCA algorithm is based on a singular value decomposition (SVD), which is explained as follows. The estimated needle tip curvature $\hat{\kappa}_{d}$ and the duty-cycle factor $\alpha$ are used to obtain the needle maximum curvature for each sub-trajectory. As shown in Fig. 5.1, to compensate for the measurement noise in needle tip curvature estimation, a Kalman Filter is used to estimate the needle tip maximum curvature $\hat{\kappa_{\max }}$. This parameter is used by the duty-cycled needle steering and motion planner for correct adaptation. 


\section{List of References}

[1] E. Demaine and S. Devadas, 6.006 Introduction to Algorithms. Fall Massachusetts Institute of Technology: MIT OpenCourseWare, License: Creative Commons BY-NC-SA, 2011. [Online]. Available: https://ocw.mit.edu

[2] C. Rossa and M. Tavakoli, "Issues in closed-loop needle steering," Control Engineering Practice, vol. 62, pp. 55-69, 2017.

[3] N. J. Cowan, K. Goldberg, G. S. Chirikjian, G. Fichtinger, R. Alterovitz, K. B. Reed, V. Kallem, W. Park, S. Misra, and A. M. Okamura, "Robotic needle steering: Design, modeling, planning, and image guidance," in Surgical robotics. Springer, 2011, pp. 557-582.

[4] D. Glozman and M. Shoham, "Flexible needle steering and optimal trajectory planning for percutaneous therapies," in International Conference on Medical Image Computing and Computer-Assisted Intervention. Springer, 2004, pp. $137-144$.

[5] V. G. Mallapragada, N. Sarkar, and T. K. Podder, "Robot-assisted real-time tumor manipulation for breast biopsy," IEEE Transactions on Robotics, vol. 25, no. 2, pp. 316-324, 2009.

[6] T. Lehmann, C. Rossa, N. Usmani, R. Sloboda, and M. Tavakoli, "A virtual sensor for needle deflection estimation during soft-tissue needle insertion," in 2015 IEEE International Conference on Robotics and Automation (ICRA). IEEE, 2015, pp. 1217-1222.

[7] T. R. Wedlick and A. M. Okamura, "Characterization of pre-curved needles for steering in tissue," in 2009 Annual International Conference of the IEEE Engineering in Medicine and Biology Society. IEEE, 2009, pp. 1200-1203.

[8] P. J. Swaney, J. Burgner, H. B. Gilbert, and R. J. Webster, "A flexure-based steerable needle: high curvature with reduced tissue damage," IEEE Transactions on Biomedical Engineering, vol. 60, no. 4, pp. 906-909, 2013. 
[9] S. P. Dimaio, "Modelling, simulation and planning of needle motion in soft tissues," Ph.D. dissertation, 2003. [Online]. Available: https://open.library.ubc.ca/collections/ubctheses/831/items/1.0065587

[10] S. P. DiMaio and S. E. Salcudean, "Interactive simulation of needle insertion models," IEEE transactions on biomedical engineering, vol. 52, no. 7, pp. 11671179, 2005.

[11] B. Williams and E. Frazzoli, 16.410 Principles of Autonomy and Decision Making. Fall Massachusetts Institute of Technology: MIT OpenCourseWare, License: Creative Commons BY-NC-SA, 2010. [Online]. Available: https://ocw.mit.edu

[12] P. Moreira, S. Patil, R. Alterovitz, and S. Misra, "Needle steering in biological tissue using ultrasound-based online curvature estimation," in 2014 IEEE International Conference on Robotics and Automation (ICRA). IEEE, 2014, pp. 4368-4373.

[13] G. J. Vrooijink, M. Abayazid, S. Patil, R. Alterovitz, and S. Misra, "Needle path planning and steering in a three-dimensional non-static environment using twodimensional ultrasound images," The International journal of robotics research, vol. 33, no. 10, pp. 1361-1374, 2014.

[14] R. J. Webster III, J. S. Kim, N. J. Cowan, G. S. Chirikjian, and A. M. Okamura, "Nonholonomic modeling of needle steering," The International Journal of Robotics Research, vol. 25, no. 5-6, pp. 509-525, 2006.

[15] S. Patil and R. Alterovitz, "Interactive motion planning for steerable needles in 3d environments with obstacles," in 2010 3rd IEEE RAS \& EMBS International Conference on Biomedical Robotics and Biomechatronics. IEEE, 2010, pp. 893899.

[16] D. S. Minhas, J. A. Engh, M. M. Fenske, and C. N. Riviere, "Modeling of needle steering via duty-cycled spinning," in 2007 29th Annual International Conference of the IEEE Engineering in Medicine and Biology Society. IEEE, 2007, pp. 2756-2759.

[17] R. Alterovitz, M. Branicky, and K. Goldberg, "Constant-curvature motion planning under uncertainty with applications in image-guided medical needle steering," in Algorithmic Foundation of Robotics VII. Springer, 2008, pp. 319-334. 
[18] S. Patil, J. Burgner, R. J. Webster, and R. Alterovitz, "Needle steering in 3d via rapid replanning," IEEE Transactions on Robotics, vol. 30, no. 4, pp. 853-864, 2014.

[19] B. Wood, F. Banovac, M. Friedman, Z. Varro, K. Cleary, J. Yanof, C. Bauer, P. Klahr, S. Gay, and Z. Neeman, "Ct-integrated programmable robot for image-guided procedures: comparison of free-hand and robot-assisted techniques," J Vasc Interv Radiol, vol. 14, p. S62, 2003.

[20] N. J. Kumar, B. George, and M. Sivaprakasam, "Virtual instrumentation system with real-time visual feedback and needle position warning suitable for ophthalmic anesthesia training," IEEE Transactions on Instrumentation and Measurement, vol. 67, no. 5, pp. 1111-1123, 2018.

[21] K. B. Reed, A. M. Okamura, and N. J. Cowan, "Modeling and control of needles with torsional friction," IEEE transactions on biomedical engineering, vol. 56, no. 12, pp. 2905-2916, 2009.

[22] J. A. Engh, G. Podnar, S. Y. Khoo, and C. Riviere, "Flexible needle steering system for percutaneous access to deep zones of the brain," in Proceedings of the IEEE 32nd annual northeast bioengineering conference. IEEE, 2006, pp. 103-104.

[23] K. B. Reed, A. Majewicz, V. Kallem, R. Alterovitz, K. Goldberg, N. J. Cowan, and A. M. Okamura, "Robot-assisted needle steering," IEEE robotics \& automation magazine, vol. 18, no. 4, pp. 35-46, 2011.

[24] M. Khadem, C. Rossa, R. S. Sloboda, N. Usmani, and M. Tavakoli, "Mechanics of tissue cutting during needle insertion in biological tissue," IEEE Robotics and Automation Letters, vol. 1, no. 2, pp. 800-807, 2016.

[25] S. M. LaValle, Planning algorithms. Cambridge university press, 2006.

[26] L. Kavraki and J.-C. Latombe, "Randomized preprocessing of configuration for fast path planning," in Proceedings of the 1994 IEEE International Conference on Robotics and Automation. IEEE, 1994, pp. 2138-2145.

[27] S. M. LaValle, "Rapidly-exploring random trees: A new tool for path planning," 1998.

[28] L. E. Kavraki, P. Svestka, J.-C. Latombe, and M. H. Overmars, "Probabilistic roadmaps for path planning in high-dimensional configuration spaces," IEEE transactions on Robotics and Automation, vol. 12, no. 4, pp. 566-580, 1996. 
[29] J. Barraquand and J.-C. Latombe, "Robot motion planning: A distributed representation approach," The International Journal of Robotics Research, vol. 10, no. 6, pp. 628-649, 1991.

[30] A. Leibinger, A. E. Forte, Z. Tan, M. J. Oldfield, F. Beyrau, D. Dini, and F. R. y Baena, "Soft tissue phantoms for realistic needle insertion: a comparative study," Annals of biomedical engineering, vol. 44, no. 8, pp. 2442-2452, 2016.

[31] N. Abolhassani, R. Patel, and M. Moallem, "Needle insertion into soft tissue: A survey," Medical engineering $\&$ physics, vol. 29, no. 4, pp. 413-431, 2007.

[32] R. J. Webster, J. Memisevic, and A. M. Okamura, "Design considerations for robotic needle steering," in Proceedings of the 2005 IEEE International Conference on Robotics and Automation. IEEE, 2005, pp. 3588-3594.

[33] I. Elgezua, Y. Kobayashi, and M. G. Fujie, "Survey on current state-of-the-art in needle insertion robots: Open challenges for application in real surgery," Procedia CIrP, vol. 5, pp. 94-99, 2013.

[34] D. Stoianovici, "Urobotics - urology robotics at johns hopkins," Computer Aided Surgery, vol. 6, no. 6, pp. 360-369, 2001.

[35] H. Koyama, T. Uchida, H. Funakubo, K. Takakura, and H. Fankhauser, "Development of a new microsurgical robot for stereotactic neurosurgery," Stereotactic and functional neurosurgery, vol. 54, no. 1-8, pp. 462-467, 1990.

[36] K. Masamune, G. Fichtinger, A. Patriciu, R. C. Susil, R. H. Taylor, L. R. Kavoussi, J. H. Anderson, I. Sakuma, T. Dohi, and D. Stoianovici, "System for robotically assisted percutaneous procedures with computed tomography guidance," Computer Aided Surgery, vol. 6, no. 6, pp. 370-383, 2001.

[37] S. P. DiMaio and S. E. Salcudean, "Needle insertion modeling and simulation," IEEE Transactions on robotics and automation, vol. 19, no. 5, pp. 864-875, 2003.

[38] M. D. O’Leary, C. Simone, T. Washio, K. Yoshinaka, and A. M. Okamura, "Robotic needle insertion: Effects of friction and needle geometry," in 2003 IEEE International Conference on Robotics and Automation (Cat. No. 03CH37422), vol. 2. IEEE, 2003, pp. 1774-1780.

[39] M. Torabi, K. Hauser, R. Alterovitz, V. Duindam, and K. Goldberg, "Guiding medical needles using single-point tissue manipulation," in 2009 IEEE International Conference on Robotics and Automation. IEEE, 2009, pp. 2705-2710. 
[40] S. Misra, K. Ramesh, and A. M. Okamura, "Modeling of tool-tissue interactions for computer-based surgical simulation: a literature review," Presence: Teleoperators and Virtual Environments, vol. 17, no. 5, pp. 463-491, 2008.

[41] R. Alterovitz, K. Goldberg, J. Pouliot, R. Taschereau, and I.-C. Hsu, "Needle insertion and radioactive seed implantation in human tissues: Simulation and sensitivity analysis," in 2003 IEEE International Conference on Robotics and Automation (Cat. No. 03CH37422), vol. 2. IEEE, 2003, pp. 1793-1799.

[42] L. Barbé, B. Bayle, M. de Mathelin, and A. Gangi, "Needle insertions modeling: Identifiability and limitations," Biomedical signal processing and control, vol. 2, no. 3, pp. 191-198, 2007.

[43] A. M. Okamura, C. Simone, and M. D. O'leary, "Force modeling for needle insertion into soft tissue," IEEE transactions on biomedical engineering, vol. 51, no. 10, pp. 1707-1716, 2004.

[44] M. Mahvash and P. E. Dupont, "Mechanics of dynamic needle insertion into a biological material," IEEE Transactions on Biomedical Engineering, vol. 57, no. 4, pp. 934-943, 2010.

[45] M. Khadem, C. Rossa, N. Usmani, R. S. Sloboda, and M. Tavakoli, "A two-body rigid/flexible model of needle steering dynamics in soft tissue," IEEE/ASME Transactions on Mechatronics, vol. 21, no. 5, pp. 2352-2364, 2016.

[46] T. R. Wedlick and A. M. Okamura, "Characterization of robotic needle insertion and rotation in artificial and ex vivo tissues," in 2012 4th IEEE RAS \& EMBS International Conference on Biomedical Robotics and Biomechatronics (BioRob). IEEE, 2012, pp. 62-68.

[47] M. Waine, C. Rossa, R. Sloboda, N. Usmani, and M. Tavakoli, "Needle tracking and deflection prediction for robot-assisted needle insertion using $2 \mathrm{~d}$ ultrasound images," Journal of Medical Robotics Research, vol. 1, no. 01, p. 1640001, 2016.

[48] C. Rossa, M. Khadem, R. Sloboda, N. Usmani, and M. Tavakoli, "Adaptive quasi-static modelling of needle deflection during steering in soft tissue," IEEE Robotics and Automation Letters, vol. 1, no. 2, pp. 916-923, 2016.

[49] N. Abolhassani, R. V. Patel, and F. Ayazi, "Minimization of needle deflection in robot-assisted percutaneous therapy," The international journal of medical Robotics and computer assisted surgery, vol. 3, no. 2, pp. 140-148, 2007. 
[50] A. Asadian, M. R. Kermani, and R. V. Patel, "An analytical model for deflection of flexible needles during needle insertion," in 2011 IEEE/RSJ International Conference on Intelligent Robots and Systems. IEEE, 2011, pp. 2551-2556.

[51] R. Alterovitz, K. Goldberg, and A. Okamura, "Planning for steerable bevel-tip needle insertion through $2 \mathrm{~d}$ soft tissue with obstacles," in Proceedings of the 2005 IEEE international conference on robotics and automation. IEEE, 2005, pp. 1640-1645.

[52] V. Duindam, R. Alterovitz, S. Sastry, and K. Goldberg, "Screw-based motion planning for bevel-tip flexible needles in 3d environments with obstacles," in 2008 IEEE international conference on robotics and automation. IEEE, 2008, pp. 2483-2488.

[53] S. P. DiMaio and S. E. Salcudean, "Needle steering and motion planning in soft tissues," IEEE Transactions on Biomedical Engineering, vol. 52, no. 6, pp. 965-974, 2005.

[54] L. E. Kavraki and J.-C. Latombe, "Probabilistic roadmaps for robot path planning," 1998.

[55] R. Alterovitz, S. Patil, and A. Derbakova, "Rapidly-exploring roadmaps: Weighing exploration vs. refinement in optimal motion planning," in 2011 IEEE International Conference on Robotics and Automation. IEEE, 2011, pp. 37063712 .

[56] V. Duindam, J. Xu, R. Alterovitz, S. Sastry, and K. Goldberg, "Threedimensional motion planning algorithms for steerable needles using inverse kinematics," The International Journal of Robotics Research, vol. 29, no. 7, pp. 789-800, 2010.

[57] J. J. Kuffner Jr and S. M. LaValle, "Rrt-connect: An efficient approach to single-query path planning," in ICRA, vol. 2, 2000.

[58] J. Xu, V. Duindam, R. Alterovitz, and K. Goldberg, "Motion planning for steerable needles in $3 \mathrm{~d}$ environments with obstacles using rapidly-exploring random trees and backchaining," in 2008 IEEE international conference on automation science and engineering. IEEE, 2008, pp. 41-46.

[59] R. Alterovitz, T. Siméon, and K. Goldberg, "The stochastic motion roadmap: A sampling framework for planning with markov motion uncertainty," in Robotics: Science and systems, 2007. 
[60] R. Alterovitz, A. Lim, K. Goldberg, G. S. Chirikjian, and A. M. Okamura, "Steering flexible needles under markov motion uncertainty," in 2005 IEEE/RSJ International Conference on Intelligent Robots and Systems. IEEE, 2005, pp. 1570-1575.

[61] D. Glozman and M. Shoham, "Image-guided robotic flexible needle steering," IEEE Transactions on Robotics, vol. 23, no. 3, pp. 459-467, 2007.

[62] Z. Neubach and M. Shoham, "Ultrasound-guided robot for flexible needle steering," IEEE Transactions on Biomedical Engineering, vol. 57, no. 4, pp. 799-805, 2009.

[63] A. Shkolnik, M. Walter, and R. Tedrake, "Reachability-guided sampling for planning under differential constraints," in 2009 IEEE International Conference on Robotics and Automation. IEEE, 2009, pp. 2859-2865.

[64] M. C. Bernardes, B. V. Adorno, P. Poignet, N. Zemiti, and G. A. Borges, "Adaptive path planning for steerable needles using duty-cycling," in 2011 IEEE/RSJ International Conference on Intelligent Robots and Systems. IEEE, 2011, pp. 2545-2550.

[65] M. Bernardes, B. V. Adorno, P. Poignet, and G. Borges, "Robot-assisted automatic insertion of steerable needles with closed-loop imaging feedback and intraoperative trajectory replanning," Mechatronics, vol. 23, no. 6, pp. 630$645,2013$.

[66] V. Kallem and N. J. Cowan, "Image-guided control of flexible bevel-tip needles," in Proceedings 2007 IEEE International Conference on Robotics and Automation. IEEE, 2007, pp. 3015-3020.

[67] R. Alterovitz, M. Branicky, and K. Goldberg, "Motion planning under uncertainty for image-guided medical needle steering," The International journal of robotics research, vol. 27, no. 11-12, pp. 1361-1374, 2008.

[68] J. Sovizi, S. Kumar, and V. Krovi, "Approximating markov chain approach to optimal feedback control of a flexible needle," Journal of Dynamic Systems, Measurement, and Control, vol. 138, no. 11, p. 111006, 2016.

[69] B. Fallahi, C. Rossa, R. S. Sloboda, N. Usmani, and M. Tavakoli, "Slidingbased switching control for image-guided needle steering in soft tissue," IEEE Robotics and Automation Letters, vol. 1, no. 2, pp. 860-867, 2016. 
[70] — - "Sliding-based image-guided 3d needle steering in soft tissue," Control Engineering Practice, vol. 63, pp. 34-43, 2017.

[71] J. Lee and W. Park, "Insertion planning for steerable flexible needles reaching multiple planar targets," in 2013 IEEE/RSJ International Conference on Intelligent Robots and Systems. IEEE, 2013, pp. 2377-2383.

[72] O. A. Bobrenkov, J. Lee, and W. Park, "A new geometry-based plan for inserting flexible needles to reach multiple targets," Robotica, vol. 32, no. 6, pp. 985-1004, 2014.

[73] E. Lobaton, J. Zhang, S. Patil, and R. Alterovitz, "Planning curvatureconstrained paths to multiple goals using circle sampling," in 2011 IEEE International Conference on Robotics and Automation. IEEE, 2011, pp. 1463-1469.

[74] J. Xu, V. Duindam, R. Alterovitz, J. Pouliot, J. A. M. Cunha, I.-C. Hsu, and K. Goldberg, "Planning fireworks trajectories for steerable medical needles to reduce patient trauma," in 2009 IEEE/RSJ International Conference on Intelligent Robots and Systems. IEEE, 2009, pp. 4517-4522.

[75] P. Moreira and S. Misra, "Biomechanics-based curvature estimation for ultrasound-guided flexible needle steering in biological tissues," Annals of biomedical engineering, vol. 43, no. 8, pp. 1716-1726, 2015.

[76] S. Misra, K. B. Reed, B. W. Schafer, K. Ramesh, and A. M. Okamura, "Mechanics of flexible needles robotically steered through soft tissue," The International journal of robotics research, vol. 29, no. 13, pp. 1640-1660, 2010.

[77] J. A. Engh, G. Podnar, D. Kondziolka, and C. N. Riviere, "Toward effective needle steering in brain tissue," in 2006 International Conference of the IEEE Engineering in Medicine and Biology Society. IEEE, 2006, pp. 559-562.

[78] A. Majewicz, J. J. Siegel, A. A. Stanley, and A. M. Okamura, "Design and evaluation of duty-cycling steering algorithms for robotically-driven steerable needles," in 2014 IEEE International Conference on Robotics and Automation (ICRA). IEEE, 2014, pp. 5883-5888.

[79] N. J. Van De Berg, T. L. De Jong, D. J. Van Gerwen, J. Dankelman, and J. J. Van Den Dobbelsteen, "The influence of tip shape on bending force during needle insertion," Scientific reports, vol. 7, p. 40477, 2017. 
[80] M. C. Bernardes, B. Adorno, P. Poignet, N. Zemiti, and G. Borges, "Path planning for steerable needles using duty-cycled spinning," in BIO Web of Conferences, vol. 1. EDP Sciences, 2011, p. 00010.

[81] T. Tan, J.-J. Mordang, J. van Zelst, A. Grivegnée, A. Gubern-Mérida, J. Melendez, R. M. Mann, W. Zhang, B. Platel, and N. Karssemeijer, "Computer-aided detection of breast cancers using haar-like features in automated 3d breast ultrasound," Medical physics, vol. 42, no. 4, pp. 1498-1504, 2015.

[82] T. H. Cormen, C. E. Leiserson, R. L. Rivest, and C. Stein, "Introduction to algorithms second edition," The Knuth-Morris-Pratt Algorithm, year, 2001.

[83] J. Suthakorn, N. Tanaiutchawoot, C. Wiratkapan, and S. Ongwattanakul, "Breast biopsy navigation system with an assisted needle holder tool and 2d graphical user interface," European journal of radiology open, vol. 5, pp. 93-101, 2018.

[84] K. B. Reed, V. Kallem, R. Alterovitz, K. Goldbergxz, A. M. Okamura, and N. J. Cowan, "Integrated planning and image-guided control for planar needle steering," in 2008 2nd IEEE RAS \& EMBS International Conference on Biomedical Robotics and Biomechatronics. IEEE, 2008, pp. 819-824.

[85] K. Hauser, R. Alterovitz, N. Chentanez, A. Okamura, and K. Goldberg, "Feedback control for steering needles through $3 \mathrm{~d}$ deformable tissue using helical paths," Robotics science and systems: online proceedings, p. 37, 2009.

[86] T. K. Adebar, J. D. Greer, P. F. Laeseke, G. L. Hwang, and A. M. Okamura, "Methods for improving the curvature of steerable needles in biological tissue," IEEE Transactions on Biomedical Engineering, vol. 63, no. 6, pp. 1167-1177, 2016 .

[87] T. K. Adebar, A. E. Fletcher, and A. M. Okamura, "3-d ultrasound-guided robotic needle steering in biological tissue," IEEE Transactions on Biomedical Engineering, vol. 61, no. 12, pp. 2899-2910, 2014.

[88] V. Kallem and N. J. Cowan, "Image guidance of flexible tip-steerable needles," IEEE Transactions on Robotics, vol. 25, no. 1, pp. 191-196, 2009.

[89] H.-W. Nienhuys and A. F. van der Stappen, "A computational technique for interactive needle insertions in 3d nonlinear material," in IEEE International Conference on Robotics and Automation, 2004. Proceedings. ICRA'04. 2004, vol. 2. IEEE, 2004, pp. 2061-2067. 
[90] M. P. Curry and A. Bonder, "Hepatomegaly: Differential diagnosis and evaluation," Up ToDate Nov, 2015.

[91] M. Patzak, M. Porzner, S. Oeztuerk, R. A. Mason, M. Wilhelm, T. Graeter, W. Kratzer, M. M. Haenle, A. S. Akinli, and E. S. Group, "Assessment of liver size by ultrasonography," Journal of Clinical Ultrasound, vol. 42, no. 7, pp. 399-404, 2014.

[92] D. C. Wolf, "Evaluation of the size, shape, and consistency of the liver," in Clinical Methods: The History, Physical, and Laboratory Examinations. 3rd edition. Butterworths, 1990.

[93] S. Shirmohammadi and A. Ferrero, "Camera as the instrument: the rising trend of vision based measurement," IEEE Instrumentation \& Measurement Magazine, vol. 17, no. 3, pp. 41-47, 2014.

[94] N. A. Wood, K. Shahrour, M. C. Ost, and C. N. Riviere, "Needle steering system using duty-cycled rotation for percutaneous kidney access," in 2010 Annual International Conference of the IEEE Engineering in Medicine and Biology. IEEE, 2010, pp. 5432-5435.

[95] D. J. Brenner and E. J. Hall, "Computed tomography - an increasing source of radiation exposure," New England Journal of Medicine, vol. 357, no. 22, pp. 2277-2284, 2007.

[96] N. Glossop, K. Cleary, and F. Banovac, "Needle tracking using the aurora magnetic position sensor," Proceedings of the International society for computer assisted orthopaedic surgery (CAOS), pp. 90-92, 2002.

[97] S. P. DiMaio, E. Samset, G. Fischer, I. Iordachita, G. Fichtinger, F. Jolesz, and C. M. Tempany, "Dynamic mri scan plane control for passive tracking of instruments and devices," in International Conference on Medical Image Computing and Computer-Assisted Intervention. Springer, 2007, pp. 50-58.

[98] P. M. Novotny, J. A. Stoll, N. V. Vasilyev, J. Pedro, P. E. Dupont, T. E. Zickler, and R. D. Howe, "Gpu based real-time instrument tracking with threedimensional ultrasound," Medical image analysis, vol. 11, no. 5, pp. 458-464, 2007.

[99] G. J. Vrooijink, M. Abayazid, and S. Misra, "Real-time three-dimensional flexible needle tracking using two-dimensional ultrasound," in 2013 IEEE International Conference on Robotics and Automation. IEEE, 2013, pp. 1688-1693. 
[100] A. Asadian, M. R. Kermani, and R. V. Patel, "Robot-assisted needle steering using a control theoretic approach," Journal of Intelligent 6 Robotic Systems, vol. 62, no. 3-4, pp. 397-418, 2011.

[101] J. op den Buijs, M. Abayazid, C. L. de Korte, and S. Misra, "Target motion predictions for pre-operative planning during needle-based interventions," in 2011 Annual International Conference of the IEEE Engineering in Medicine and Biology Society. IEEE, 2011, pp. 5380-5385.

[102] J. op den Buijs, H. H. Hansen, R. G. Lopata, C. L. de Korte, and S. Misra, "Predicting target displacements using ultrasound elastography and finite element modeling," IEEE Transactions on Biomedical Engineering, vol. 58, no. 11, pp. 3143-3155, 2011.

[103] N. N. Stone, J. Roy, S. Hong, Y.-C. Lo, and R. G. Stock, "Prostate gland motion and deformation caused by needle placement during brachytherapy," Brachytherapy, vol. 1, no. 3, pp. 154-160, 2002.

[104] P. Sears and P. Dupont, "A steerable needle technology using curved concentric tubes," in 2006 IEEE/RSJ international conference on intelligent robots and systems. IEEE, 2006, pp. 2850-2856. 